LIVIA FERNANDA ARAUJO BORGES

An embedded discrete fracture model based on the use of coupling finite elements for modeling fluid flow in naturally fractured porous media 
LIVIA FERNANDA ARAUJO BORGES

An embedded discrete fracture model based on the use of coupling finite elements for modeling fluid flow in naturally fractured porous media

Dissertation presented to the Polytechnic School at the University of São Paulo as a requirement for obtaining the Master's Degree in Science. 


\section{An embedded discrete fracture model based on the use of coupling finite elements for modeling fluid flow in naturally fractured porous media}

Revised Version

Dissertation presented to the Polytechnic School at the University of São Paulo as part of the requirement for obtaining the Master's Degree in Science.

Area of Concentration: Geotechnical Engineering

Advisor: Prof. Dr. Luís A. G. Bitencourt Jr.

Co-Advisor: Prof. Dr. Osvaldo L. Manzoli 
Autorizo a reprodução e divulgação total ou parcial deste trabalho, por qualquer meio convencional ou eletrônico, para fins de estudo e pesquisa, desde que citada a fonte.

Este exemplar foi revisado e corrigido em relação à versão original, sob responsabilidade única do autor e com a anuência de seu orientador.

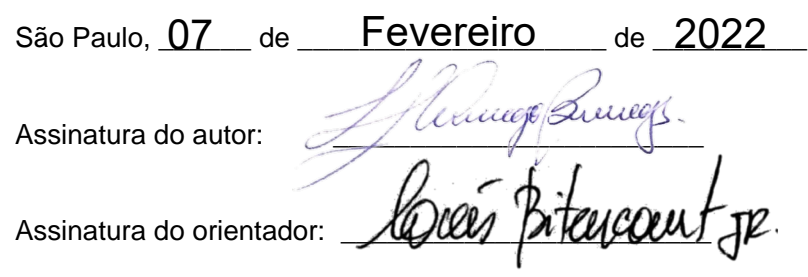

Borges, Livia Fernanda Araujo

An embedded discrete fracture model based on the use of coupling finite elements for modeling fluid flow in naturally fractured porous media / L. F. A. Borges -- versão corr. -- São Paulo, 2022.

$87 \mathrm{p}$.

Dissertação (Mestrado) - Escola Politécnica da Universidade de São Paulo. Departamento de Engenharia de Estruturas e Geotécnica.

1.Embedded discrete fracture 2.Non-matching meshes 3.Coupling finite elements 4.Naturally fractured porous media 5.Fluid flow I.Universidade de São Paulo. Escola Politécnica. Departamento de Engenharia de Estruturas e Geotécnica II.t. 


\section{Acknowledgments}

This dissertation was developed in context of the research project "Computational modeling of the hydromechanical behavior of naturally fractured and karstified reservoirs via mesh fragmentation technique" sponsored by the Petróleo Brasileiro S.A. (Petrobras) - grant number: 2018/00205-5. The company's support is gratefully acknowledged. I also would like to acknowledge that this study was financed in part by the Coordenação de Aperfeiçoamento de Pessoal de Nível Superior - Brasil (CAPES) - Finance Code 001.

I would like to express all my gratitude to my family, specially my parents Ligia Berti and Mauro Borges. They were the base I need most, the people who never give up on me and always gave me the freedom to choose the paths I wanted to walk. Thank you very much for the advice and the support in my entire life, I would never have finished this work without you. Equally important in my life are my cousins who always were an example to follow for me since I was a little child, thank you very much for everything, Luciana and Daniel Ziroldo.

I also would like to express my deep gratitude to my advisor, Prof. Luís A. G. Bitencourt Jr. for his support, encouragement and advice during this research. I also wish to thank him for never had any doubt about my capacity and for all the opportunities he gave to me to effort on different research projects.

I also wish to thank my co-advisor, Prof. Osvaldo Luís Manzoli, for his interest in my research and assistance. The opportunity to work with your research group was essential to finish this dissertation.

I would like to express my sincere thanks and appreciation to the Computational Modeling in Solid Mechanics Group (GMCMS), specially Pedro Cleto, Michael Maedo and Eduardo Rodrigues for always been available to help me out in any issue that I had.

To the colleagues, I made in the Laboratory of Structures and Materials (LEM) my

appreciation for the friendship we made (Maria Luísa Mineiro, Paulo Victor Gama 
and André Silva), even when we were not able to meet due to Covid-19 pandemic you were there with supporting words, specially Guilherme Gianotti and Ludmily Pereira.

I want to thank the first friends I made when I came to São Paulo, and have been on this journey with me since the beginning, Amanda Steff, Jéssica Santos and Luana Mendes. 


\section{Abstract}

Petroleum is a natural occurrence of hydrocarbons and inorganic impurities, usually presented in a liquid or gas phase in a system. Some reservoirs rock which stores the hydrocarbon can be separated between two domains: the porous medium and the fracture network which may creates preferential channels that change the equivalent permeability of the medium. Many numerical models have been proposed to simulate fluid flow in naturally fractured reservoirs, however, the representation of the complex geometric characteristics of the fracture network is still an issue nowadays. In this sense, this work proposes an embedded discrete fracture model based on the use of coupling finite elements (CFEs) for modeling fluid flow in naturally fractured porous media. The proposed model is able to couple the initially independent non-matching and overlapping meshes from the fractures and rock matrix via CFEs to enforce the continuity of the pressure field between the meshes through a penalty parameter. The main advantage of the use of this approach is that the implementation of CFEs does not require additional degrees of freedom or special integration procedures for coupling the non-matching meshes. Three sets of 2D numerical examples are performed to validate the proposed approach for modeling the steady-state flow in fractured porous medium. The first set of numerical examples focused on the influence of the natural fractures position and the penalty parameter magnitude. In the second set of examples, a study of mesh refinement is performed considering fracture intersections in order to assess the capability of the proposed model to account for the contribution of each discontinuity to the pressure field. The last set of numerical examples presents the simulation of a complex naturally fractured porous medium with vug pore spaces. In conclusion with the results obtained: (i) the coarse meshes presents good results in comparison with refined meshes; (ii) a ratio of porous media and fracture elements between 1 and 2 is acceptable for better results; and (iii) and an specific value of the penalty parameter is applied to predict better behavior of fluid flow in porous medium. Furthermore, the results are in good agreement with reference solutions (the numerical results obtained in 
previous studies) and the proposed approach demonstrated to be able to capture the main phenomena associated with the fluid transport in fractured porous media. Keywords: Embedded discrete fracture; Non-matching meshes; Coupling finite element; Naturally fractured porous media; Fluid flow. 


\section{Resumo}

Petróleo é uma ocorrência natural de hidrocarbonetos e impurezas inorgânicas, geralmente presente em fase líquida ou gasosa em um sistema. Algumas rochas reservatório que alocam os hidrocarbonetos podem ser divididas em dois domínios: o meio poroso e a rede de fraturas que cria canais preferenciais de fluxo que altera a permeabilidade equivalente do meio. Vários modelos numéricos têm sido propostos para simular o fluxo de fluido em reservatórios naturalmente fraturados, no entanto, a representação de características complexas da geometria da rede de fraturas ainda é um desafio nos dias de hoje. Neste contexto, este trabalho propõe um modelo de fraturas discretas embebidas baseado na utilização de elementos finitos de acoplamento (EFA) para modelar fluxo de fluido em meios porosos naturalmente fraturados. O modelo proposto é capaz de acoplar inicialmente as malhas independentes não conformes e sobrepostas da fratura e da matriz rochosa via EFAs para impor a continuidade do campo de pressão entre as malhas por meio de um parâmetro de penalidade. A principal vantagem na utilização dessa técnica é que a implementação dos EFAs não requer graus de liberdade adicionais ou processos de integração especiais para acoplar as malhas não conformes. Três conjuntos de exemplos numéricos 2D são performados a fim de validar o esquema proposto para modelar fluxo de fluido hidrostático em meios porosos fraturados. O primeiro conjunto de exemplo numérico é focado na influência da posição das fraturas naturais e na magnitude do parâmetro de penalidade. No segundo conjunto de exemplos, um estudo de refinamento de malha é feito considerando intersecções de fraturas a fim de estudar a capacidade do modelo proposto de contabilizar a contribuição de cada descontinuidade no campo de pressão. O último conjunto de exemplos apresenta a simulação de um complexo meio poroso naturalmente fraturado contendo vugs. A conclusão dos resultados obtidos são: (i) as malhas grosseiras apresentam bons resultados em comparação com as malhas refinadas; (ii) a razão dos elementos do meio poroso e da fratura entre 1 e 2 é aceitável para melhores resultados; e (iii) um valor específico do parâmetro de penalidade é aplicado para prever um melhor 
comportamento do fluxo de fluido no meio poroso. Além disso, os resultados obtidos estão em boa concordância com as soluções de referência (os resultados numéricos obtidos em estudos anteriores) e o esquema proposto demonstra habilidade para capturar os principais fenômenos associados com o transporte de fluido em meios porosos fraturados.

Palavras-chave: Fraturas discretas embebidas; malhas não conformes; elemento finito de acoplamento; meios porosos naturalmente fraturados; fluxo de fluido. 


\section{List of Figures}

1.1 Schematic conceptual model of a petroleum system with conventional and unconventional production of hydrocarbons (adapted from Byrne et al. (2018)) . . . . . . . . . . . . . . . . . . 2

1.2 Conceptual models of a porous medium proposed by Li (2017): (a) with large and small fractures; (b) with complex network fracture and vugs interconnected; (c) with vugs connected to large fractures; and (d) with vugs isolated of fractures. . . . . . . . . . . . . 3

1.3 Types of fractured porous medium and conceptual models used in the analysis of fluid pressure distribution (adapted from Dietrich et al., 2005). . . . . . . . . . . . . . . . . . . 4 4

1.4 Conceptual continuum and discrete methods and their level of realistic representation of fracture and porous medium domains (adapted from Berre et al., 2019). . . . . . . . . . . . . . . . . . . . 6

2.1 Representative elementary volume (REV) of a porous medium and porosity representation according to the portion of volume estimated (adapted from Lie and Mallison (2013)). . . . . . . . . . . . . 10

2.2 Darcy's experiment of fluid flow through an inclined sand filter. . . . 10

2.3 Schematic representation of the Parallel Plate Model composed of two walls of fractures separated by an aperture. . . . . . . . . . . . 13

2.4 Mass conservation for a REV . . . . . . . . . . . . . . . . 15

2.5 Fractured porous medium (left) and and details of a fracture domain (right) . . . . . . . . . . . . . . . . . . 17 
4.1 Schematic representation of the 2D coupling strategy for non-matching meshes: (a) porous matrix and fractures geometries; (b) mesh discretization of the porous matrix (three-noded triangular elements) and fracture (two-noded bar elements) domains; (c) Insertion of CFEs (four-noded triangular elements) to couple the meshes from porous medium and fracture. . . . . . . . . . . . . . . . . . 23

4.2 CFE - Four-noded triangular finite element. . . . . . . . . . . . 25

5.1 Vertical single fracture problem: (a) geometry and boundary conditions; (b) Reference mesh; (c) Mesh 1 (structured mesh); (d) Mesh 2 (unstructured and coarse mesh); (e) Mesh 3 (unstructured and intermediate mesh) and (f) Mesh 4 (unstructured and fine mesh). . . . . . 28

5.2 Pressure distribution of the single vertical fracture problem: (a) Reference; (b) Mesh 1; (c) Mesh 2; (d) Mesh 3; and (e) Mesh 4. . . . . . 30

5.3 Pressure profile of the single vertical fracture problem at: (a) $y_{1}=$ $0 \mathrm{~m}$; (b) $y_{2}=0.2 \mathrm{~m}$; and (c) $y_{3}=0.5 \mathrm{~m} \ldots \ldots 31$

5.4 RMS error curves for the pressure profile crossing at $y_{2}=0.2 \mathrm{~m}$. . . 32

5.5 Single diagonal fracture problem: (a) boundary conditions applied

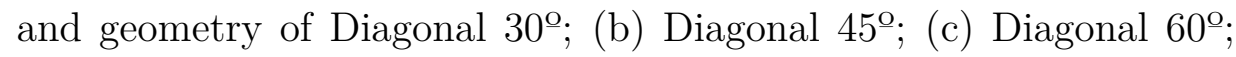
(d) Horizontal 90ㅜㅇ and (e) Diagonal 135ㅇ․ . . . . . . . . . . . . 33

5.6 Equidimensional and conforming meshes used in the numerical analyses of the single diagonal fracture problem: (a) Diagonal 30\%; (b) Diagonal 45º; (c) Diagonal 60º; (d) Horizontal 90º; and (e) Diagonal $135^{\mathrm{o}} \ldots \ldots \ldots \ldots \ldots$

5.7 Conforming meshes used in the numerical analysis of the single diago-

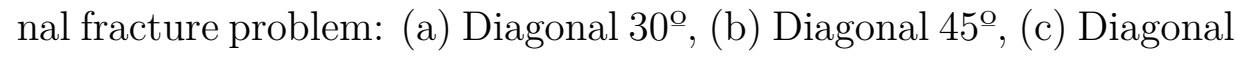
$60^{\circ}$, (d) Horizontal $90^{\circ}$ and (e) Diagonal 135º . . . . . . . . . . . . . 34

5.8 Meshes with CFEs in the numerical analysis of the single diagonal fracture problem: (a) Diagonal 30º; (b) Diagonal 45ㅜ; (c) Diagonal $60^{\mathrm{o}}$; (d) Horizontal 90º ; and (e) Diagonal 135ㅇ․ . . . . . . . . . . . . 35

5.9 Pressure field for the single diagonal fracture problem: (a) Equidimensional mesh; (b) Conforming mesh; and (c) CFEs mesh. . . . . . 37

5.10 Pressure profile for the three case studies of the single diagonal fracture problem along $y=0.5 \mathrm{~m}$ : (a) Diagonal $30^{\circ}$; (b) Diagonal $45^{\circ}$; (c) Diagonal 60º ; (d) Horizontal 90º ; and (e) Diagonal 135ㅇ․ . . . . . 38 
5.11 Pressure profile for the 15 meshes considered in the numerical analyses of the single diagonal fracture problem at $y=0.5 \mathrm{~m} . \quad \ldots . . .39$

5.12 Intersected fractures problem: (a) geometry and boundary conditions; (b) details of fracture intersection for each mesh; (c) Reference mesh; (d) Coarse mesh (matching fractures with intersection node); (e) Coarse mesh (non-matching fractures with overlapped intersection node); (e) Coarse mesh (non-matching fractures with no intersection node); (f) Intermediate mesh (non-matching fractures with no intersection node); and (h) Refined mesh (non-matching fractures with no intersection node). . . . . . . . . . . . . . . . 41

5.13 Pressure gradient of the intersected fractures problem: (a) Reference mesh; (b) Coarse mesh (matching fractures with intersection node); (c) Coarse mesh (non-matching fractures with overlapped intersection node); (d) Coarse mesh (non-matching fractures with no intersection node); (e) Intermediate mesh (non-matching fractures with no intersection node); and (f) Refined mesh (non-matching fractures with no intersection node). . . . . . . . . . . . . . . . . . 43

5.14 Pressure profile crossing the intersection of fractures at $y=1 \mathrm{~m}$ of the intersected fracture problem.

5.15 Regular fracture network: (a) geometry and boundary conditions and (b) cut lines related to the problem. . . . . . . . . . . . 45

5.16 FE meshes used for the Case 1 of the regular fracture network problem: (a) Mesh 1; (b) Mesh 2; (c) Mesh 3; and (d) Mesh 4. . . . . . . 47

5.17 FE meshes used for the Case 2 of the regular fracture network problem: (a) Mesh 5; (b) Mesh 6; (c) Mesh 7; and (d) Mesh 8. . . . . . . 48

5.18 FE meshes used for the Case 3 of the Regular fracture network problem: (a) Mesh 9; (b) Mesh 10; (c) Mesh 11; and (d) Mesh 12. . . . . . 49

5.19 Pressure gradient obtained for the regular fracture network for: (a) Case 1, (b) Case 2 and (c) Case 3. . . . . . . . . . . . . . . . 50

5.20 Comparison between the results presented by Flemisch et al. (2018) against that ones via CFEs related to the Case 1 measured in (a) $x=0.5 \mathrm{~m}$ and (b) $y=0.7 \mathrm{~m}$; Case 2 measured in (c) $x=0.5 \mathrm{~m}$ and (d) $y=0.7 \mathrm{~m}$, and Case 3 measured in (e) $x=0.5 \mathrm{~m}$ and (f) $y=0.7 \mathrm{~m} . \quad 52$

5.21 Pressure profile of regular fracture network with CFEs and the methods studied by Flemisch et al. (2018). . . . . . . . . . . . . . . 53 
5.22 RMS error between the reference curve presented by Flemisch and the results obtained via CFE method related to the Cases 1, 2 and 3. 54

5.23 Geometry and boundary conditions for the three cases considered: (a) fractured porous medium; (b) vuggy porous medium; and (c) fractured-vuggy porous medium. . . . . . . . . . . . . . 55

5.24 FE mesh discretization with CFEs of conceptual models: (a) fractured porous medium; (b) vuggy porous medium; and (c) fracturedvuggy porous medium. . . . . . . . . . . . . . 56

5.25 Pressure distribution of (a) fractured porous medium; (b) vuggy porous medium; and (c) vuggy-fractured porous medium. . . . . . . . . . 56

5.26 Pressure profile for the three cases numerically analyzed along $y=10 \mathrm{~m} .58$ 


\section{List of Tables}

3.1 Components of the three-noded triangular and two-noded bar elements. 20

5.1 Features of the FE meshes employed for the numerical simulations of the single vertical fracture problem. . . . . . . . . . . . . 29

5.2 Characteristics of the FE meshes employed for the numerical simulations of the single diagonal fracture problem. . . . . . . . . . . 36

5.3 RMS error values for the 5 cases studied. . . . . . . . . . . . . 39

5.4 Characteristics of the FE meshes employed for the numerical simulations of the intersected fracture problem. . . . . . . . . . . . . 42

5.5 Properties of the meshes employed for the regular fracture network

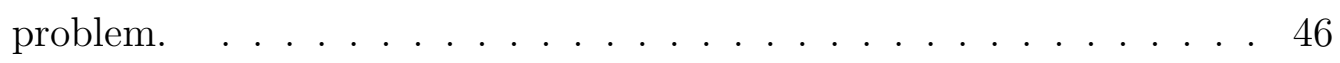

5.6 Characteristics of the FE meshes used in the numerical models. . . . 57 


\section{Contents}

1 Introduction 1

1.1 General aspects and motivation . . . . . . . . . . . . . . 1

1.2 Discrete fracture models . . . . . . . . . . . . . . 6

1.3 Objectives ........................ 7

1.4 Structure of the dissertation . . . . . . . . . . . . . 8

2 Governing equations 9

2.1 Main concepts of porous medium and fracture domains . . . . . . . 9

2.1.1 Darcy's law for fluid flow in porous medium . . . . . . . . . 9

2.1.2 Cubic Law and Parallel Plate Model for fluid flow in fracture . 12

2.2 Fluid flow in porous medium and fracture domains . . . . . . . . . . 14

2.2.1 Continuity equation of fluid for a nondeformable representative elementary volume . . . . . . . . . . . . . 14

2.2.2 Fluid balance equations of fluid flow in porous medium and fracture domains . . . . . . . . . . . . . . . . . 16

3 Modeling fluid flow in porous medium and fracture domains with finite $\begin{array}{lr}\text { element method } & 18\end{array}$

3.1 Weak form of governing equations . . . . . . . . . . . . . . 18

3.2 Finite element discretization . . . . . . . . . . . . . . . . . . 19

4 Interaction between the FE meshes from porous medium and fractures 22

4.1 Overview of the procedure for coupling non-matching meshes . . . . . 22

4.2 Coupling finite element discretization . . . . . . . . . . . . . 24

4.3 Fluid exchange vector and permeability matrix . . . . . . . . 25

5 Numerical analyses $\quad 27$

5.1 Validation of the proposed model . . . . . . . . . . . . 28

5.1 .1 Single vertical fracture . . . . . . . . . . . . . . . . . . . . . . . . . . . 32

5.1 .2 Single diagonal fracture . . . . . . . . . . . . . 32 
5.2 Mesh refinement study . . . . . . . . . . . . . . . . . . . . . 39

5.2 .1 Intersected fractures $\ldots \ldots \ldots \ldots \ldots$

5.2 .2 Regular fracture network . . . . . . . . . . . . . . . 44

5.3 Naturally fractured-vuggy reservoirs $\ldots \ldots \ldots \ldots \ldots \ldots$

6 Conclusions $\quad 59$

6.1 General conclusions . . . . . . . . . . . . . . . . . . 59

6.2 Recommendations for future research . . . . . . . . . . . 60

$\begin{array}{ll}\text { Bibliography } & 62\end{array}$ 


\section{Introduction}

\subsection{General aspects and motivation}

The oil industry is one of the most important sectors in the world economy, responsible to feed energy matrices and to provide raw materials for multiple products used in the daily routine of people. Petroleum, the principal material, is a natural occurrence of hydrocarbons and inorganic impurities, usually presented in a liquid or gas phase in a system (Lyons and Plisga, 2011).

The petroleum system consists of different geological components and processes able to generate and accumulate hydrocarbons in certain temperature and pressure conditions (Dandekar, 2013; Fanchi and Christiansen, 2017). Understanding these processes allows forecasting where the accumulation of hydrocarbons is expected to happen. In general, conventional resources are the most used petroleum system to extract and produce oil and gas. However, unconventional resources gained particular importance in the petroleum industry due to the increased demand for hydrocarbons in the last decades (see Chengzao et al. (2012); Vedachalam et al. (2015); Liu et al. (2017); Zhang et al. (2018)).

The exploration prospect of the two systems acts in different manners. On the one hand, the conventional resources have a favorable structural and stratigraphic trap that allows the formation of hydrocarbons on a source rock sealed by an impermeable layer. The source rock is connected to a migration path where the fluid moves away to the reservoir rock, able to store hydrocarbons in its pores (Alyafei, 2019). On the other hand, in unconventional resources, tight formations induce the source and the reservoir rock to coexist in the same space, and parameters like porosity and permeability are stated as ultra-low (Song et al., 2015; Ma and Holditch, 2015). Although extraction of hydrocarbons is a demanding process in both system, the unconventional resource covers a great area of petroleum exploration, as for example, the pre-salt layers from Brazilian Santos and Campos basins, which are one of the 
biggest oil and gas petroleum system discovered in the world (Leffler et al., 2011). Figure 1.1 illustrates a schematic conceptual model of the sources for production of hydrocarbons.

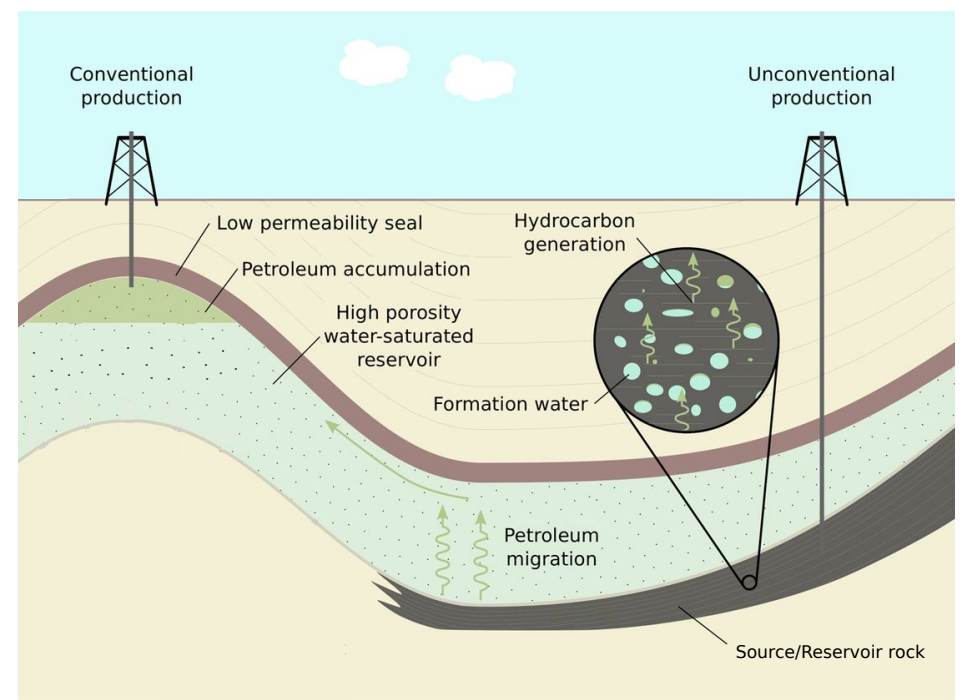

Figure 1.1: Schematic conceptual model of a petroleum system with conventional and unconventional production of hydrocarbons (adapted from Byrne et al. (2018)).

The reservoir rock, a fundamental formation of the unconventional petroleum systems, consists of many pore spaces filled with oil, gas, or water. An important characteristic to measure the fluid storage capacity in the rock is porosity. In general, effective porosity creates pathways responsible for the flow of hydrocarbons (Etnyre, 1989). When the pores are well connected, the transport of fluids is given from one pore to another, creating preferential pathways for fluid flow, and to measure the capacity of fluid transport inside these interconnected pores, a parameter called permeability is used (Leffler et al., 2011). Reservoir rock like shale and carbonate with a great porosity but poorly connected may become a reservoir if it is geologically classified as naturally fractured (Thomas, 2004). In the case of carbonate reservoirs, a variety of classifications according to physical and geological characteristics can be made, such as cave reservoirs, vuggy reservoirs, fractured reservoirs, and fractured-vuggy reservoirs (Li, 2017).

Carbonate reservoirs are complex systems featured by a heterogeneous and diverse space. These heterogeneities are often a reflection of the complex association be- 
tween microstructures, especially carbonate minerals that experienced the diagenesis process over time (Regnet et al., 2019). As a consequence of this process, the reservoir is occupied by natural fractures and the pore space, which may be composed of karst formations that induce a higher permeability on the porous medium (Tiab and Donaldson, 2015; Li, 2017). The geometry of natural fractures (size, aperture and aspect ratio) affects physical rock properties and plays an important role in the heterogeneity of carbonate reservoirs, considering that permeability of fractures intends to greatly differ from the permeability of the porous medium (Chen et al., 2020; Fabbri et al., 2021). Meanwhile, the pore space, characterized by its size, shape and connectivity, also influences rock parameters, e.g., vuggy space provides storage of fluids and, directly or indirectly, interacts with fracture network (Wu et al., 2011).

According to the geometrical distribution of the vuggy portion, fracture network and porous medium, some distribution patterns can be found. Figure 1.2 demonstrates conceptual models of reservoirs and the interaction among matrix, fractures and vugs.

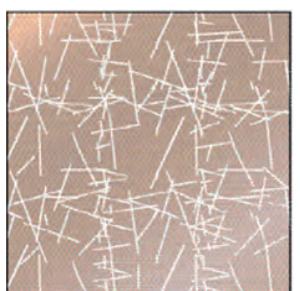

(a)

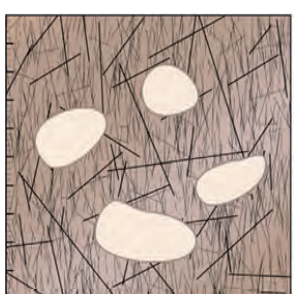

(b)

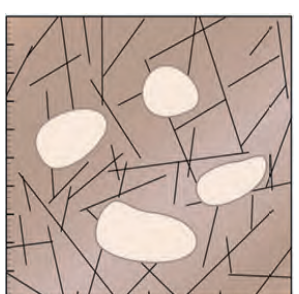

(c)

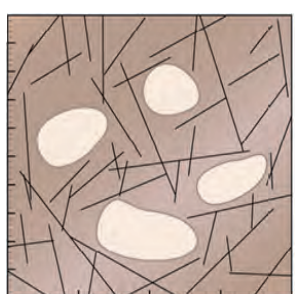

(d)

Figure 1.2: Conceptual models of a porous medium proposed by Li (2017): (a) with large and small fractures; (b) with complex network fracture and vugs interconnected; (c) with vugs connected to large fractures; and (d) with vugs isolated of fractures.

Based on the above considerations, it is noticeable the importance of research development based on predict flow behavior in fractured porous medium in an accurate manner for petroleum industry. As consequence, after the advent of computers and with more computational capability available in the last decades, several numerical models have been proposed for modeling fluid flow in a naturally fractured porous medium. In general, these models can be grouped into three principal categories 
(Fang et al., 2017; Liu et al., 2019): (1) equivalent continuum fracture models; (2) dual-continuum models; and (3) discrete fracture methods. Figure 1.3 illustrates the types of fractured porous medium and the concept models usually applied for each case according to their characteristics.
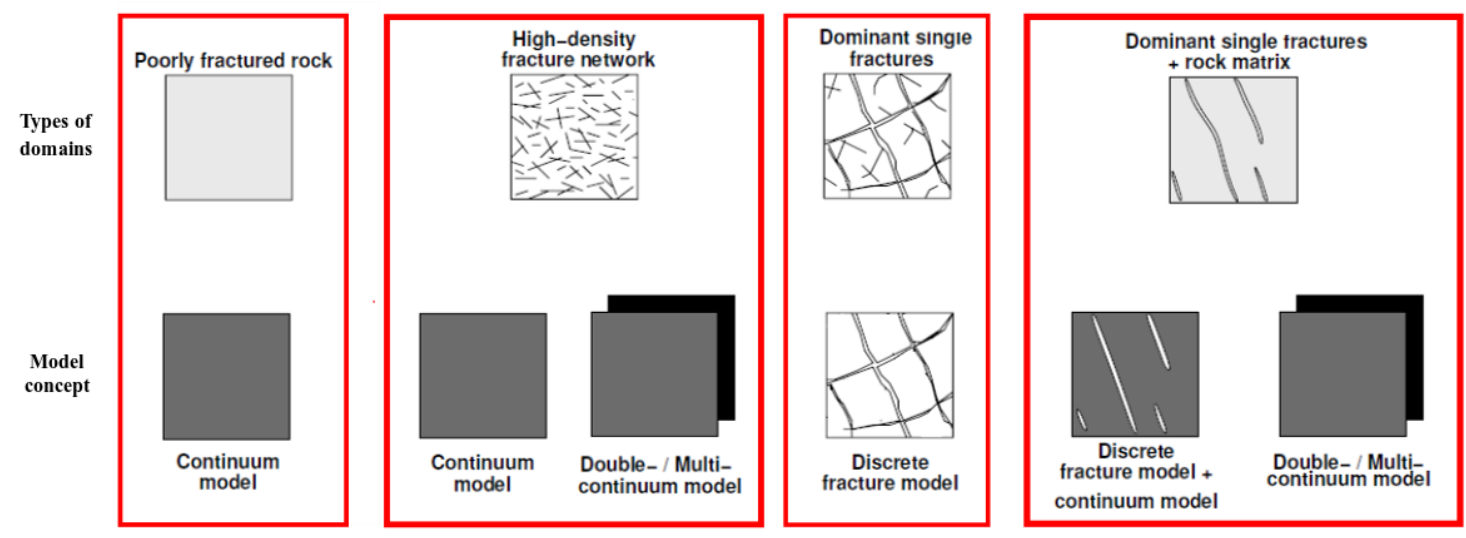

Figure 1.3: Types of fractured porous medium and conceptual models used in the analysis of fluid pressure distribution (adapted from Dietrich et al., 2005).

In the equivalent continuum fracture models, a unique equivalent matrix-fracture system is considered (Long et al., 1982; Coats et al., 1989). Even nowadays, continuum models can be used to treat upscaling problems of reservoir with a uniform fracture network by adopting different approaches such as Multiscale Finite Volume (MSFV) methods (Hajibeygi et al., 2014; Sokolova et al., 2019), Nonlocal Multicontinua (NLMC) methods (Chung et al.; Vasilyeva et al., 2019) and Generalized Multiscale Finite Element Methods (GMsFEM) (Efendiev et al., 2013; Spiridonov et al., 2019; Presho and Hill, 2020). However, it is important to mention that models of this group do not consider the geometric differences between the porous matrix and the complex fracture system, and parameters like porosity or permeability are measured by the average behavior of the whole domain. Another limitation is to evaluate, accurately, the transfer functions applied to the interaction between porous matrix and fractures (Karimi-Fard et al., 2003; Moinfar et al., 2011; Huang et al., 2019).

The dual-continuum models (dual-porosity and dual permeability) assume that porous medium and fractures can be represented as independent continua, which are connected through a transfer function (Warren et al., 1963; Kazemi et al., 1976; Moench, 1984). Warren et al. (1963) firstly introduced the double-porosity model for a fractured porous medium assuming fluid transport between fracture to matrix and fracture to fracture. Kazemi et al. (1976) advanced the model for naturally 
fractured reservoirs developing a mathematical formulation for two-phase flow. Hill et al. (1985) proposed a double-porosity/double-permeability model for fractures and porous medium considering the system as an interacting continuum. This model is able to treat mass transfer between two segments of the matrix. Another option to characterize the reservoir heterogeneity in dual-continuum models is made using triple porosity models, which considers either two sets of fractures and a porous medium or two sets of porous medium and fractures (Abdassah et al., 1986; Aguilera et al., 2004; Al-Ahmadi et al., 2011). In the same line of research, other general methods were proposed to solve problems of fluid flow in a fractured porous medium as the multiple interacting continua (MINC) (Pruess and Narasimhan, 1982) and multi-porosity models (Wu et al., 1988; Yan et al., 2016a). Models of this group also present limitations in terms of realistic representation of complex geometry of fractures (Moinfar et al., 2011).

In an effort to overcome the drawbacks presented by continuum models, discrete methods have emerged as an interesting alternative. In the discrete methods, the fractures are represented individually and explicitly with their flux exchange in the surrounding porous domain. (Cundall and Hart, 1992; Damirchi et al., 2020). Figure 1.4 presents a comparison between continuum and discrete models due to accuracy in the representation of fracture and porous matrix. Despite the accuracy of the discrete models to represent individually and explicitly the fractures with their flux exchange in the surrounding porous domain, standard discrete models are limited to matching meshes between the porous medium and fractures (Jiang and Younis, 2017), i.e., fracture and porous medium elements share the nodes at the boundary interfaces between these subdomains. This mesh characteristic can be feasible for a problem with a single fracture, but cumbersome for a fracture network (Manzoli et al., 2021). In this sense, more recently, discrete models based on a non-matching mesh discretization have been proposed to avoid the need for mesh transitions between the porous matrix and fractures. In these models, fractures and porous domains are generated independently, and the elements of fractures can cross the porous medium at any point material (Yan et al., 2016b; Fang et al., 2017). Consequently, models of this group require a technique to connect the initially independent meshes from the porous matrix and fractures, as for instance, Lagrange multipliers (Wohlmuth, 2001; Dhia and Rateau, 2005; Schädle et al., 2019), discontinuous Galerkin methods (Březina and Exner, 2017) and Nitsche methods (Annavarapu et al., 2012; Becker et al., 2003). 


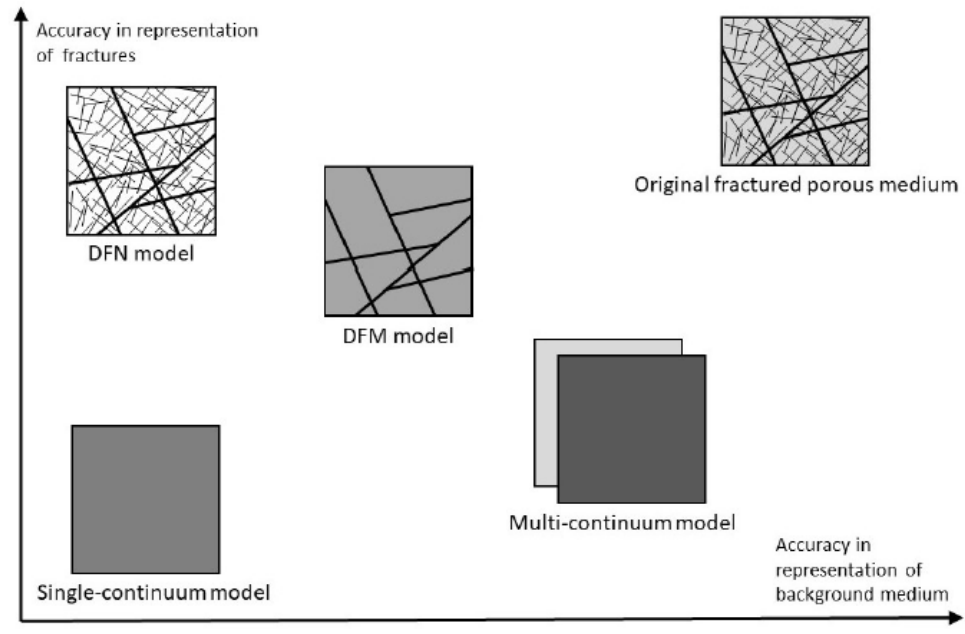

Figure 1.4: Conceptual continuum and discrete methods and their level of realistic representation of fracture and porous medium domains (adapted from Berre et al., 2019).

In the following, given the context of this research and the importance nowadays of the discrete fracture models for modeling fluid flow in naturally fractured porous media, a brief description of the models available in the literature is presented.

\subsection{Discrete fracture models}

Several discrete fracture models using non-matching models have been proposed. In this context, embedded discrete fracture models (EDFM) are among the most used approaches to address the mass transport of fluid in fractured formations. Chai et al. (2018) extended the EDFM to consider the mass transfer between fractures and matrix with multiple porosity/permeability setting. The matrix is sub-divided into three porosity types and the macro-fractures are explicitly represented with EDFM. The approach provides desirable accuracy for the simulation of fractured reservoir considering 2D problems. Wang et al. (2019) used EDFM to treat hydraulic fractures in 3D reservoir simulation. In their work, a vertex-based geometric algorithm is developed to handle discretization errors caused by local domain resolution, although the method is not optimized to simulate a fluid in transient state crossing a large size matrix with low permeability. Xu et al. (2020) extended the EDFM for unstructured grid using element-based finite volume method (EbFVM), a method based on finite elements and the finite volume method that enhances non-matching discretization and maintains local mass conservation. The method was adopted 
to treat fracture networks in 2D and 3D hydraulic problems. Rao et al. (2020) enhanced the method for projection-based EDFM (pEDFM) to simulate blocking fractures in a reservoir. The method is able to solve 2D problems, although, for 3D simulations the fracture planes projected require a lot of interactions increasing the complexity of the problem. In the framework of EDFM and fluid flow in porous media, Damirchi et al. (2020) used 2D coupling finite elements to couple the matrix and fracture meshes, which is able to deal with the fluid interaction between both domains. However, problems involving intersecting fractures were not studied. Moreover, the method proposed by Damirchi et al. (2020) requires that the nodes of the fracture mesh must be on the edges of the matrix elements.

Despite the extensive applications of techniques using EDFM, this models discretize the domain in cells and it requires the calculation of three types of transmissibility factors for fluid exchange: (i) one between fracture and matrix, (ii) one between the segments of the same fracture inside a cell and (iii) another between different fractures intersecting each other. Moreover, EDFM exhibits a higher computational cost when complex fracture distributions are analyzed ( $\mathrm{Li}$ et al., 2020).

This research presents a new approach based on the use of coupling finite elements (CFEs) to consider the hydraulic effect of discrete fractures in the simulation of fluid transport in naturally fractured porous media. The main objective is to extend the formulation for coupling non-matching meshes developed by Bitencourt Jr et al. (2015) in the context of mechanical problems (Bitencourt Jr et al., 2018; Rodrigues et al., 2018; Bitencourt Jr et al., 2019; Rodrigues et al., 2020; Trindade et al., 2020) to tackle problems involving fluid flow in $2 \mathrm{D}$ porous media with complex discrete fracture networks. Thus, the proposed approach allows the independent discretization of fractures and matrix (overlapping non-matching meshes) without increasing the total number of degrees of freedom of the problem. Moreover, this technique can be easily included in the existing FEM codes in a pre-processing stage.

\subsection{Objectives}

The main objective of this dissertation is to propose a new discrete fracture model based on the use of coupling finite elements (CFEs) to simulate steady-state fluid flow in naturally fractured porous media. Furthermore, the specifics goals of this research are listed below: 
- To extend the formulation for coupling non-matching meshes developed by Bitencourt Jr et al. (2015) in the context of mechanical problems to tackle problems involving steady-state fluid flow in 2D porous media with complex discrete fracture networks.

- To investigate the mesh refinement influence on the pressure field.

- To assess the influence of the value of the coupling parameter on the results.

- To verify the ability of the proposed approach for modeling fluid flow in porous media with complex fracture systems.

\subsection{Structure of the dissertation}

This dissertation is organized in 6 chapters. The first chapter presents the general aspects about naturally fractured reservoir for the petroleum industry and the motivation of this research. A brief literature review of the methods available to simulate fluid flow in porous media with discontinuities and the objectives of this research are also presented in this chapter. Chapter 2 addresses the governing equations of flow in fractured porous medium. The finite element approximation is developed to solve the hydraulic problem in chapter 3 . In chapter 4 the coupling finite element formulation and the main aspects to represent discontinuities in a porous matrix are detailed. Results and discussions are presented in chapter 5. Finally, the general conclusions are given in chapter 6 . 


\section{Governing equations}

\subsection{Main concepts of porous medium and fracture domains}

A porous medium is a solid with void space that creates connected pores throughout the medium. Usually, the solid portion is impermeable and the pore portion is filled with one or several fluids. On the one hand, each phase of the porous medium can be considered as a homogeneous material on a microscopic scale, and the behavior of a single fluid in a unique pore is easily deduced. On the other hand, the prediction of fluid behavior on a large scale is a challenging task due to the heterogeneity of porous medium in macroscale (Bear, 1972; Wu, 2016). As consequence, some considerations using a continuum approach on the macroscopic level can be considered. For example, the porous medium is conceived as a continuous space and described by its average properties (Bear and Bachmat, 1990).

The representative elementary volume (REV) is a concept largely used in practice, which is able to define rock and fluid parameters when it is assembled with a local equilibrium assumption. Figure 2.1 demonstrates a REV with a satisfactory portion of volume to represent the parameters of the principal materials.

\subsubsection{Darcy's law for fluid flow in porous medium}

In the study of homogeneous fluid flow, Darcy (1856) included an experimental test of water flow through sand filters. Figure 2.2 illustrates Darcy's experiment for an inclined sand filter.

The results concluded that the total volume of water per unit time $(Q)$ is given by:

$$
Q=K A \frac{\left(h_{1}-h_{2}\right)}{L}
$$




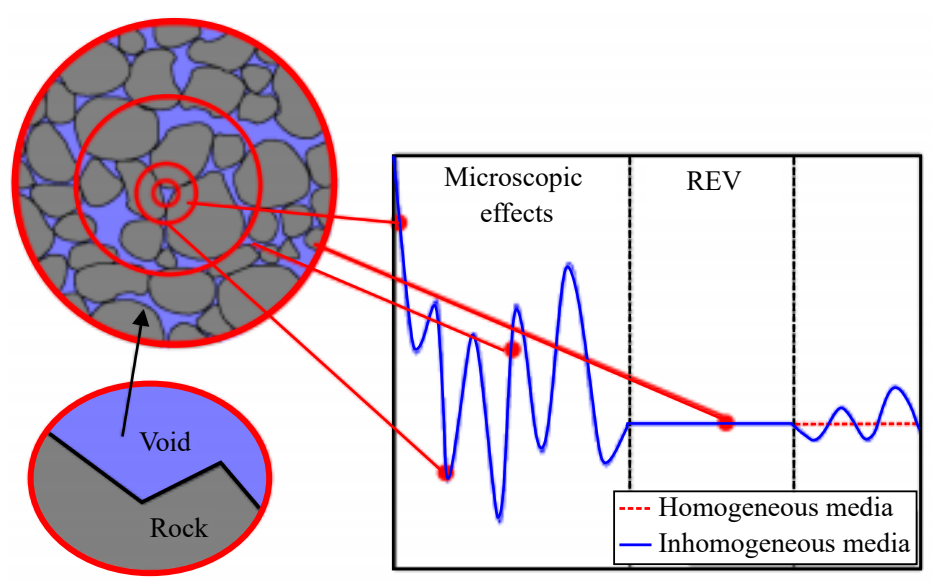

Figure 2.1: Representative elementary volume (REV) of a porous medium and porosity representation according to the portion of volume estimated (adapted from Lie and Mallison (2013)).

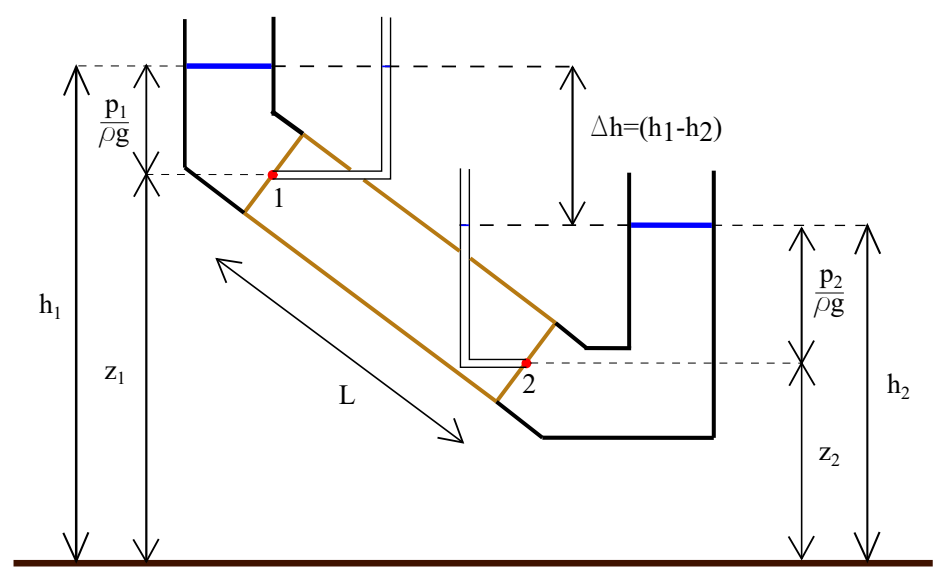

Figure 2.2: Darcy's experiment of fluid flow through an inclined sand filter.

Eq. 2.1 is called Darcy's law, where $L$ and $A$ are the filter length and cross-sectional area, respectively; $K$ is the hydraulic conductivity of porous medium; and $\left(h_{1}-h_{2}\right)$ is the difference of piezometric head across the filter column. The equation which describes the piezometric head is composed by a pressure head $(\psi)$ and an elevation head $(z)$, as follows:

$$
h=\psi+z=\frac{p}{\rho g}+z
$$

where $p$ is the pressure; $\rho$ is the fluid density; and $g$ is the gravity acceleration. Replacing the difference of piezometric head terms using the Eq. 2.2, the Eq. 2.1 can 
be written as:

$$
Q=K A\left[\frac{1}{\rho g}\left(\frac{p_{1}-p_{2}}{L}\right)+\left(\frac{z_{1}-z_{2}}{L}\right)\right]
$$

The flow can also be described in terms of a specific discharge per unit-area $\left(q_{m}=\frac{Q}{A}\right)$ :

$$
q_{m}=K\left[\frac{1}{\rho g}\left(\frac{p_{1}-p_{2}}{L}\right)+\left(\frac{z_{1}-z_{2}}{L}\right)\right]
$$

Writing the Eq. 2.4 in a generalized manner and in terms of Cartesian coordinates $(x, y, z)$, the specific discharge vector has the form:

$$
\mathbf{q}_{\mathbf{m}}=-\frac{k}{\mu}[\nabla p-\rho \mathbf{g}]
$$

where $\nabla p$ is the pressure gradient and gis the vector of gravity acceleration. The hydraulic conductivity tensor K can be described as (Bear, 1972):

$$
\mathbf{K}=\mathbf{k}_{\mathbf{m}} \frac{\rho g}{\mu}=\left[\begin{array}{ccc}
k_{x} & 0 & 0 \\
0 & k_{y} & 0 \\
0 & 0 & k_{z}
\end{array}\right] \frac{\rho g}{\mu} .
$$

The fluid viscosity is expressed by $\mu$ and $\mathbf{k}_{\mathbf{m}}$ is the permeability matrix of porous medium with $k_{x}, k_{y}$ and $k_{z}$ components. For an isotropic porous medium, $\mathbf{k}_{\mathbf{m}}$ is given by:

$$
\mathbf{k}_{\mathbf{m}}=k_{m}\left[\begin{array}{ccc}
1 & 0 & 0 \\
0 & 1 & 0 \\
0 & 0 & 1
\end{array}\right]=k_{m} \mathbf{I}
$$

where $k_{m}$ is the permeability of porous medium and $\mathbf{I}$ is the identity matrix.

Replacing Eq. 2.6 into Eq. 2.5, the specific discharge is written as:

$$
\mathbf{q}_{\mathbf{m}}=-\frac{\mathbf{k}_{\mathbf{m}}}{\mu}\left(\boldsymbol{\nabla} p_{m}-\rho \mathbf{g}\right)
$$

where the pressure gradient of porous matrix corresponds to $\boldsymbol{\nabla} p_{m}$, and $\mathbf{g}=-\mathrm{g} \mathbf{1 z}$ is the vector of gravity acceleration, which can be neglected for the cases where it 
is perpendicular to the fluid flow plane. Therefore, Eq. 2.8 can be simplified as:

$$
\mathbf{q}_{\mathbf{m}}=-\frac{\mathbf{k}_{\mathbf{m}}}{\mu} \nabla p_{m}
$$

The specific discharge derived from Darcy's law can also be related to the fluid velocity through the porous medium (Coussy, 2004), as follows:

$$
\mathbf{q}_{\mathbf{m}}=\eta\left(\mathbf{v}_{\mathbf{m}}-\mathbf{w}\right)
$$

where, $\eta$ is the porosity; $\mathbf{v}_{\mathbf{m}}$ is the fluid velocity vector crossing porous medium; and $\mathbf{w}$ is the solid velocity vector of the fluid phase due to the displacement of solid material in the initial configuration. However, for a nondeformable porous medium w can be neglected (Bear, 1972), and Eq. 2.10 assumes the final form:

$$
\mathbf{q}_{\mathrm{m}}=\eta \mathbf{v}_{\mathbf{m}}
$$

\subsubsection{Cubic Law and Parallel Plate Model for fluid flow in fracture}

It is widely known that fractures have a great influence in porous medium and, usually, are the preferential path of fluid flow. Due to the challenge of determining the aperture of an entire length of fracture, the parallel plate model is used to simplify the solution in many cases of fractured porous medium. In this context, the model may considers the aperture a constant parameter over the fracture domain (Li et al., 2008; Liu et al., 2016). The schematic model in the Cartesian coordinate system is illustrated in Figure 2.3(a), where two smooth parallel plates are considered the opening walls of a single fracture. The fracture planes are separated by an aperture $a$ with top and bottom coordinates lying the fracture at $\pm \frac{a}{2}$ in the perpendicular direction ( $z$-axis).

Considering that the volume inside the fracture does not vary with $x$ or $y$ and that the pressure does not act in the $y$ and $z$ direction, the pressure gradient $\left(\nabla p=\frac{p_{1}-p_{2}}{l}\right)$ acting within the fracture plane over the $x$-direction is considered uniform, and is simplified for $\nabla p=\frac{\partial p}{\partial x}$. Zimmerman and Bodvarsson (1996) proposed that the flow rate is considered only in $x$-direction and depends of $z$-direction with value 0 when $z= \pm \frac{a}{2}$ and maximum value when $z=0$. Figure 2.3(b) illustrates the flow rate profile in $x z$-plane for a single fracture. 


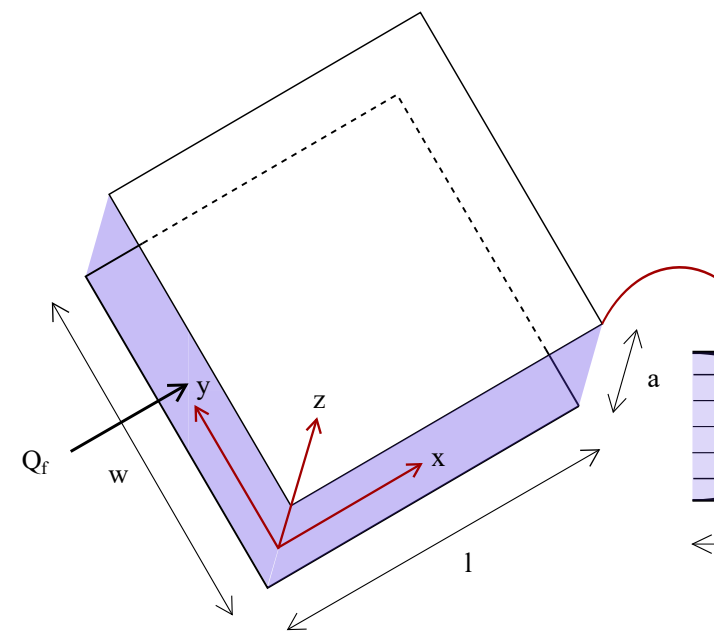

(a)

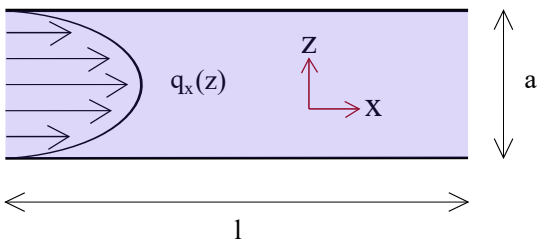

(b)

Figure 2.3: Schematic representation of the Parallel Plate Model composed of two walls of fractures separated by an aperture.

Considering a laminar flow the equation which describes the flow rate in a material point inside the fractures plane has the form (Zimmerman and Bodvarsson, 1996):

$$
q_{x}(z)=\frac{\nabla p_{f}}{2 \mu}\left[z^{2}-\left(\frac{a}{2}\right)^{2}\right]
$$

where $\nabla p_{f}$ is the pressure gradient of fracture; $\mu$ is the fluid viscosity; $z$ stands for the local coordinate of the material point and $a$ is the fracture aperture that separate the parallel plates.

To obtain the total volume per unit time $\left(Q_{f}\right)$ within a fracture of width $w$ in $y$ direction, i.e. the perpendicular direction of pressure gradient, Eq. 2.12 is integrated over the fracture aperture in $z$-direction, as follows:

$$
Q_{f}=w \int_{-\frac{a}{2}}^{+\frac{a}{2}} q_{x}(z) d z=-\frac{w a^{3} \nabla p_{f}}{12 \mu}
$$

Eq. 2.13 is known as cubic law and is used to obtain the average specific discharge per unit area within the fracture, in the following manner

$$
q_{f}=\frac{Q_{f}}{w h}=-\frac{a^{2} \nabla p_{f}}{12 \mu}
$$


where $w$ stands for the cross-sectional area of the fracture walls. Considering a isothermal and steady-state fluid flow with no mass exchange between fracture and porous medium, it is possible to note that $q_{f}$ is similar to $q_{m}$ derived from Darcy's law (Eq. 2.9). Hence, $q_{f}$ can be written analogously to Darcy's law and assumes the form

$$
q_{f}=-\frac{k_{f}}{\mu} \nabla p_{f}
$$

where the permeability of fracture, $k_{f}$, can be related to the fracture aperture through the cubic law, as follows:

$$
k_{f}=\frac{a^{2}}{12}
$$

\subsection{Fluid flow in porous medium and fracture domains}

\subsubsection{Continuity equation of fluid for a nondeformable representative elementary volume}

Consider a representative elementary volume (REV) of $d x, d y$ and $d z$ dimensions, parallel, respectively, to the Cartesian coordinate system $(x, y, z)$. The mass flux of a fluid, i.e., the mass per unit area per unit time, is given by vector $\mathbf{J}$ with $J_{x}$, $J_{y}$ and $J_{z}$ components applied to the boundary surfaces, that originate the REV, during a period of time $d t$. The influx and outflux of each direction are illustrated in Figure 2.4 and the mass variation corresponds to the difference between the influx and outflux terms, as follow:

[Mass flux incoming $]-[$ Mass flux outgoing $]=[$ Difference of mass flux $]$

The mass flux outgoing in $\mathrm{x}$ direction, $J_{x, \text { out }}$, is expressed in terms of mass flux incoming by a simplifying hypothesis for linear flow, and has the form:

$$
J_{x, o u t}=J_{x, \text { in }}+\frac{\partial J_{x}}{\partial x} d x
$$

also used to express $J_{y, \text { out }}$ and $J_{z, \text { out }}$. Replacing the outflux terms of each component 


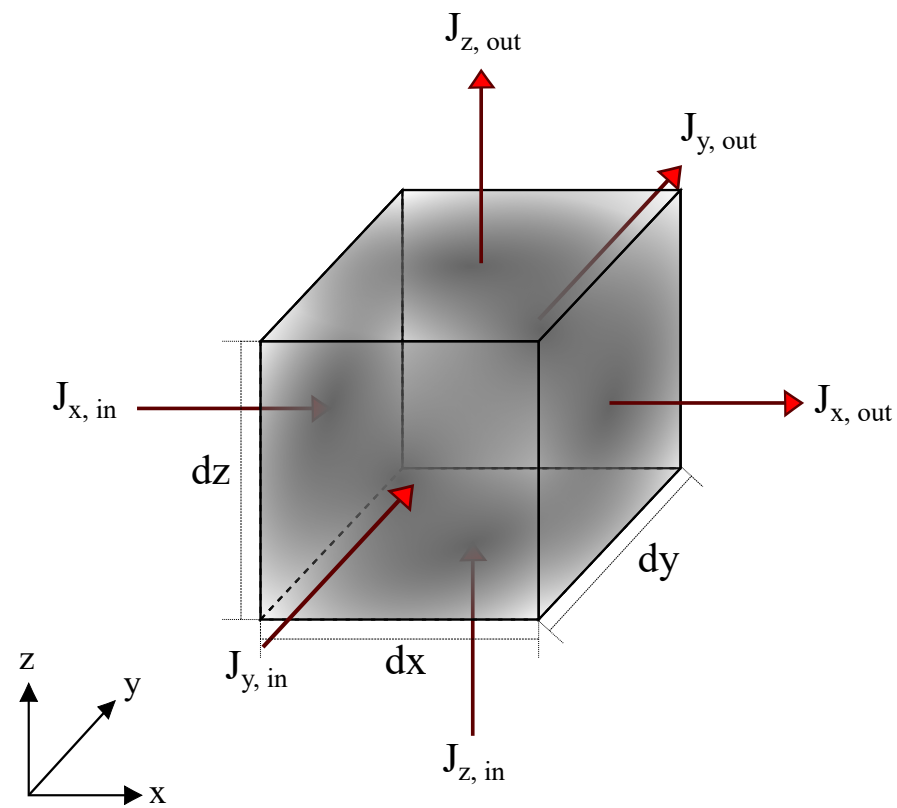

Figure 2.4: Mass conservation for a REV.

in the left side of Eq. 2.17 and considering the conservation of mass, i.e., the difference of mass flux within the REV (Bear, 1972), Eq. 2.17 turns to:

$$
-\left[\frac{\partial J_{x}}{\partial x}+\frac{\partial J_{y}}{\partial y}+\frac{\partial J_{z}}{\partial z}\right] d x d y d z d t=\frac{\partial(\eta \rho \Delta U)}{\partial t} d t
$$

where $\frac{\partial}{\partial t}$ is the partial derivative over time; $\eta$ is the porosity; $\rho$ refers to density of fluid, and $\Delta U=d x d y d z$ is the control volume.

Thus, for a nondeformable medium, i.e. $\eta=$ constant, the continuity equation of fluid is given by:

$$
-\left[\frac{\partial J_{x}}{\partial x}+\frac{\partial J_{y}}{\partial y}+\frac{\partial J_{z}}{\partial z}\right]=\frac{\eta \partial \rho}{\partial t} .
$$

The mass flux components can be written in terms of gradient vector $(\boldsymbol{\nabla} \cdot \mathbf{J})$ and Eq. 2.20 is simplified to:

$$
\frac{\eta \partial \rho}{\partial t}+\nabla \cdot \mathbf{J}=0
$$

According to Coussy (2004), the mass flux vector can be written as $\mathbf{J}=\rho \eta \mathbf{v}$, where 
$\mathbf{v}$ is the velocity vector of fluid. As consequence, Eq. 2.21 is expressed as:

$$
\frac{\eta \partial \rho}{\partial t}+\nabla \cdot(\rho \eta \mathbf{v})=0
$$

In practical problems, the spatial variation of fluid density $(\nabla \rho)$ is much smaller than the variation of fluid density over time $\left(\frac{\partial \rho}{\partial t}\right)$ and can be neglected. Then, Eq. 2.22 is simplified to:

$$
\frac{\eta \partial \rho}{\partial t}+\rho \nabla \cdot(\eta \mathbf{v})=0
$$

Besides, this work considers that fluid properties do not change over time, i.e. $\frac{\partial \rho}{\partial t}=$ 0. Thus, the Eq. 2.23 is given by

$$
\boldsymbol{\nabla} \cdot(\eta \mathbf{v})=0
$$

Therefore, considering the Eq. 2.11, the final form of continuity equation of fluid is given by the specific discharge, as follows:

$$
\nabla \cdot \mathbf{q}=0
$$

\subsubsection{Fluid balance equations of fluid flow in porous medium and fracture domains}

The continuity equation of fluid takes into account the mass conservation given by the specific discharge (Eq. 2.25). Consider a porous matrix domain $\Omega_{m} \in \mathbb{R}^{2}$ where a discrete fracture is represented by a lower dimensional domain, i.e. $\Gamma_{f} \in \mathbb{R}^{1}$, so that $\Omega_{f}=\Gamma_{f} h$, where $h$ is the fracture aperture, as illustrated in Figure 2.5.

Therefore, taking into account that the porous medium and fracture domains are initially independent, the continuity equations for these two systems can be written as:

$$
\begin{aligned}
& \boldsymbol{\nabla} \cdot\left(-\frac{\mathbf{k}_{\mathbf{m}}}{\mu} \boldsymbol{\nabla} p_{m}\right)=0 \text { in } \Omega_{m} \\
& \boldsymbol{\nabla} \cdot\left(-\frac{k_{f}}{\mu} \boldsymbol{\nabla} p_{f}\right)=0 \text { in } \Omega_{f}
\end{aligned}
$$




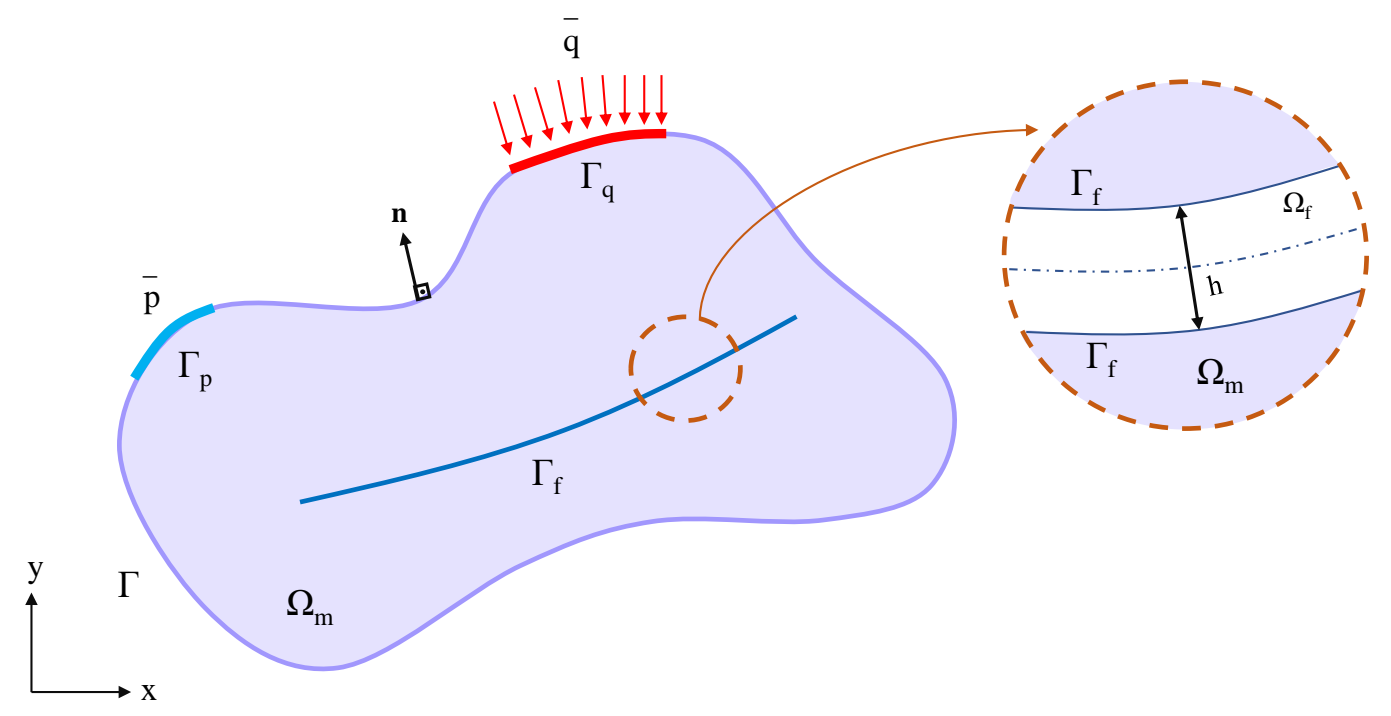

Figure 2.5: Fractured porous medium (left) and and details of a fracture domain (right).

To solve these strong forms of mass conservation of fluid in porous medium and fracture domains $\Omega_{m}$ and $\Omega_{f}$, respectively, (Eq. 2.26 and Eq. 2.27), the following boundary conditions are considered:

$$
\begin{gathered}
p_{m, f}(\mathbf{X})=\bar{p}_{m, f} \quad \mathbf{X} \in \Gamma_{p_{m, f}} \\
\mathbf{q}_{m, f}(\mathbf{X}) \cdot \mathbf{n}=\bar{q}_{m, f} \quad \mathbf{X} \in \Gamma_{q_{m, f}}
\end{gathered}
$$

where $\bar{p}_{m, f}$ and $\bar{q}_{m, f}$ are the pore pressure and specific discharge prescribed on the boundaries $\Gamma_{p_{m, f}}$ and $\Gamma_{q_{m, f}}$, respectively; $\mathbf{n}$ is the unit outward vector normal to $\Gamma$; and $\mathbf{X}$ stands for the material point. 


\section{Modeling fluid flow in porous medium and fracture domains with finite element method}

This chapter presents the development of the weak form of the governing equations from the porous medium and fracture domains and their finite element discretizations. Initially, as a non-matching mesh scheme is used in the discretization of the problem, the mathematical formulations of the two systems (i.e. the porous medium and fractures) are developed separately. Therefore, it is important to mention that the fluid exchange between the matrix and fractures are considered into the formulation of the mesh coupling scheme presented in the next chapter.

\subsection{Weak form of governing equations}

The weak form of the differential equations is equivalent to the governing equation of fluid and boundary conditions of the problem, i.e. the strong form (Jacob and Ted, 2007). Thus, considering initially the balance equation of the porous matrix (Eq. 2.26), the development of its weak form starts by multiplying this strong form by an arbitrary function $w_{m}$, (also known as weight function or test function) and integrating over its domain, such as $p \in U^{p}$ :

$$
\int_{\Omega_{m}} w_{m} \boldsymbol{\nabla} \cdot \mathbf{q}_{\mathbf{m}} d \Omega_{m}=0, \forall w \in U_{0}^{p}
$$

where

$$
\begin{aligned}
& U^{p}=p \mid p \in H^{1}, p=\bar{p} \text { in } \Gamma_{p} \\
& U_{0}^{p}=w \mid w \in H^{1}, w=0 \text { in } \Gamma_{p}
\end{aligned}
$$

In the equations above, $U^{p}$ denotes the set of all functions that are admissible trial solutions; $U_{0}^{p}$ denotes the set of all admissible weight functions; and $H^{1}$ corresponds to the space of square integrable functions. 
Applying the product rule in Eq. 3.1, it can be rewritten as:

$$
\int_{\Omega_{m}} \nabla \cdot\left(w_{m} \mathbf{q}_{\mathbf{m}}\right) d \Omega_{m}-\int_{\Omega_{m}} \nabla w_{m} \cdot \mathbf{q}_{\mathbf{m}} d \Omega_{m}=0 .
$$

Subsequently, the divergence theorem is applied in the first term of Eq. 3.2 and can be written in terms of boundary surface $\Gamma_{q m}$, as follows:

$$
\int_{\Gamma_{q_{m}}} \mathbf{n} \cdot\left(w_{m} \mathbf{q}_{\mathbf{m}}\right) d \Gamma_{q_{m}}-\int_{\Omega_{m}} \nabla w_{m} \mathbf{q}_{\mathbf{m}} d \Omega_{m}=0
$$

The first and second terms of Eq. 3.3 can be replaced for the natural boundary condition of the problem, $\mathbf{q}_{\mathbf{m}} \cdot \mathbf{n}=\bar{q}_{m}$ in $\Gamma_{q m}$, and for Darcy's law Eq. 2.9, respectively, and the weak form of governing equation for the porous medium is finally given by:

$$
\int_{\Omega_{m}} \nabla w_{m} \cdot\left(\frac{\mathbf{k}_{\mathbf{m}}}{\mu} \nabla p_{m}\right) d \Omega_{m}+\int_{\Gamma_{q_{m}}} w_{m} \bar{q}_{m} d \Gamma_{q_{m}}=0 .
$$

Following the same procedure developed for the porous matrix, the weak form of the governing equation for the fracture domain can be written as:

$$
\int_{\Omega_{f}} \nabla w_{f} \cdot\left(\frac{k_{f}}{\mu} \nabla p_{f}\right) d \Omega_{f}+\int_{\Gamma_{q_{f}}} w_{f} \bar{q}_{f} d \Gamma_{q_{f}}=0 .
$$

\subsection{Finite element discretization}

Considering the Galerkin method, the pore pressure fields $\left(p_{m, f}\right)$, test functions $\left(w_{m, f}\right)$, and their respective gradients $\left(\nabla p_{m, f}\right.$ and $\left.\boldsymbol{\nabla} w_{m, f}\right)$ at any point inside of an element " $e$ " of the porous medium " $m$ " or fracture " $f$ " can be written as:

$$
\begin{gathered}
p_{m, f}(\mathbf{X}) \approx p_{m, f}^{e}(\mathbf{X})=\mathbf{N}_{\mathbf{m}, \mathbf{f}}^{\mathbf{e}} \mathbf{p}_{\mathbf{m}, \mathbf{f}}^{\mathbf{e}} \\
\nabla p_{m, f}(\mathbf{X}) \approx \nabla \mathbf{N}_{\mathbf{m}, \mathbf{f}}^{\mathbf{e}} \mathbf{p}_{\mathbf{m}, \mathbf{f}}^{\mathbf{e}}=\mathbf{B}_{\mathbf{m}, \mathbf{f}}^{\mathbf{e}} \mathbf{p}_{\mathbf{m}, \mathbf{f}}^{\mathbf{e}} \\
w_{m, f}(\mathbf{X}) \approx w_{m, f}^{e}(\mathbf{X})=\mathbf{N}_{\mathbf{m}, \mathbf{f}}^{\mathbf{e}} \mathbf{w}_{\mathbf{m}, \mathbf{f}}^{\mathbf{e}} \\
\nabla w_{m, f}(\mathbf{X}) \approx \nabla \mathbf{N}_{\mathbf{m}, \mathbf{f}}^{\mathbf{e}} \mathbf{w}_{\mathbf{m}, \mathbf{f}}^{\mathbf{e}}=\mathbf{B}_{\mathbf{m}, \mathbf{f}}^{\mathbf{e}} \mathbf{w}_{\mathbf{m}, \mathbf{f}}^{\mathbf{e}} \\
\mathbf{X} \in \Omega^{e}
\end{gathered}
$$

where $\mathbf{X}$ are the coordinates of any point inside of an element in terms of the global Cartesian coordinate system; $\mathbf{N}_{\mathbf{m}, \mathbf{f}}^{\mathbf{e}}$ are the standard shape function matrices; $\mathbf{p}_{\mathbf{m}}^{\mathbf{e}}$ represents the vector of node pressures; $\mathbf{B}_{\mathbf{m}}^{\mathbf{e}}$ is the standard matrix containing 
the spatial derivatives of the corresponding shape functions; and $\mathbf{w}_{\mathbf{m}}^{\mathbf{e}}$ is the vector of nodal test functions. In this work, porous medium is considered $\Omega_{m} \in \mathbb{R}^{2}$ and the discrete fracture represented by a lower dimensional domain, i.e. $\Gamma_{f} \in$ $\mathbb{R}^{1}$. Therefore, the porous medium and fractures are discretized in finite elements using three-noded triangular elements and two-noded bar elements, respectively. Table 3.1 presents the main components of these finite elements.

Table 3.1: Components of the three-noded triangular and two-noded bar elements.

\begin{tabular}{|c|c|c|c|}
\hline $\begin{array}{c}\text { Element } \\
\text { Type }\end{array}$ & $\mathbf{N}^{\mathbf{e}}$ matrix & $\mathbf{B}^{\mathbf{e}}$ matrix & $\begin{array}{c}\text { Nodal } \\
\text { pressure } \\
\text { vector }\end{array}$ \\
\hline $\begin{array}{c}\text { Three- } \\
\text { noded } \\
\text { triangular } \\
\text { element }\end{array}$ & $\mathbf{N}_{\mathbf{m}}^{\mathbf{e}}=\left[\begin{array}{c}N_{m(1)} \\
N_{m(2)} \\
N_{m(3)}\end{array}\right]^{T}$ & $\mathbf{B}_{\mathbf{m}}^{\mathbf{e}}=\left[\begin{array}{lll}\frac{\partial N_{m(1)}}{\partial x} & \frac{\partial N_{m(2)}}{\partial x} & \frac{\partial N_{m(3)}}{\partial x} \\
\frac{\partial N_{m(1)}}{\partial y} & \frac{\partial N_{m(2)}}{\partial y} & \frac{\partial N_{m(3)}}{\partial y}\end{array}\right]$ & $p_{m}^{e}=\left[\begin{array}{c}p_{m(1)} \\
p_{m(2)} \\
p_{m(3)}\end{array}\right]$ \\
\hline $\begin{array}{c}\text { Two-noded } \\
\text { bar } \\
\text { elements }\end{array}$ & $\mathbf{N}_{\mathbf{f}}^{\mathbf{e}}=\left[\begin{array}{c}N_{f(1)} \\
N_{f(2)}\end{array}\right]^{T}$ & $\mathbf{B}_{\mathbf{f}}^{\mathbf{e}}=\left[\begin{array}{cc}\frac{\partial N_{f(1)}}{\partial x} & \frac{\partial N_{f(2)}}{\partial x} \\
\frac{\partial N_{f(1)}}{\partial y} & \frac{\partial N_{f(2)}}{\partial y}\end{array}\right]$ & $p_{f}^{e}=\left[\begin{array}{c}p_{f(1)} \\
p_{f(2)}\end{array}\right]$ \\
\hline
\end{tabular}

Considering the Eq. 3.6, it is possible to rewrite the weak forms of the porous matrix (Eq.3.4) and fracture domain (Eq. 3.5) as follows:

$$
\Lambda_{e=1}^{n_{e l, m}}\left[\mathbf{w}_{\mathbf{m}}^{\mathbf{e}^{\mathbf{T}}}\left(\int_{\Omega_{m}} \mathbf{B}_{\mathbf{m}}^{\mathbf{e}^{\mathbf{T}}} \frac{\mathbf{k}_{\mathbf{m}}}{\mu} \mathbf{B}_{\mathbf{m}}^{\mathbf{e}} d \Omega_{m} \mathbf{p}_{\mathbf{m}}^{\mathbf{e}}+\int_{\Gamma_{q_{m}}} \mathbf{N}_{\mathbf{m}}^{\mathbf{e}^{\mathbf{T}}} \bar{q}_{m} d \Gamma_{q_{m}}\right)\right]=0
$$

and

$$
\Lambda_{e=1}^{n_{e l, f}}\left[\mathbf{w}_{\mathbf{f}}^{\mathbf{e}^{\mathbf{T}}}\left(\int_{\Omega_{f}} \mathbf{B}_{\mathbf{f}}^{\mathbf{e}^{\mathbf{T}}} \frac{k_{f}}{\mu} \mathbf{B}_{\mathbf{f}}^{\mathbf{e}} d \Omega_{f} \mathbf{p}_{\mathbf{f}}^{\mathbf{e}}+\int_{\Gamma_{q_{f}}} \mathbf{N}_{\mathbf{f}}^{\mathbf{e}} \bar{q}_{f} d \Gamma_{q_{f}}\right)\right]=0
$$

where $\Lambda_{e=1}^{n_{e l}}$ stands for the FE assembly operator, and $n_{e l}$ is the number of porous medium $\left(n_{e l}=n_{e l, m}\right)$ or fracture $\left(n_{e l}=n_{e l, f}\right)$ elements. Since the test function $\mathbf{w}_{\mathbf{m}, \mathbf{f}}^{\mathbf{e}}$ is arbitrary and cannot be zero, these equations assume the form:

$$
\Lambda_{e=1}^{n_{e l, m}}\left(\int_{\Omega_{m}} \mathbf{B}_{\mathbf{m}}^{\mathbf{e}^{\mathbf{T}}} \frac{\mathbf{k}_{\mathbf{m}}}{\mu} \mathbf{B}_{\mathbf{m}}^{\mathbf{e}} d \Omega_{m} \mathbf{p}_{\mathbf{m}}^{\mathbf{e}}+\int_{\Gamma_{q_{m}}} \mathbf{N}_{\mathbf{m}}^{\mathbf{e}^{\mathbf{T}}} \bar{q}_{m} d \Gamma_{q_{m}}\right)=0
$$

and

$$
\Lambda_{e=1}^{n_{e l, f}}\left(\int_{\Omega_{f}} \mathbf{B}_{\mathbf{f}}^{\mathbf{e}^{\mathbf{T}}} \frac{k_{f}}{\mu} \mathbf{B}_{\mathbf{f}}^{\mathbf{e}} d \Omega_{f} \mathbf{p}_{\mathbf{f}}^{\mathbf{e}}+\int_{\Gamma_{q_{f}}} \mathbf{N}_{\mathbf{f}}^{\mathbf{e}} \bar{q}_{f} d \Gamma_{q_{f}}\right)=0
$$


where the global permeability matrix, and the external fluid flow vector for the porous matrix and fracture domains can be defined as:

$$
\begin{aligned}
\mathbf{K}_{\mathbf{m}} & =\Lambda_{e=1}^{n_{e l, m}}\left(\int_{\Omega_{m}} \mathbf{B}_{\mathbf{m}}^{\mathbf{e}^{\mathbf{T}} \frac{\mathbf{k}_{\mathbf{m}}}{\mu}} \mathbf{B}_{\mathbf{m}}^{\mathbf{e}} d \Omega_{m}\right) \\
\mathbf{Q}_{\mathbf{m}} & =\Lambda_{e=1}^{n_{e l, m}}-\left(\int_{\Gamma_{q_{m}}} \mathbf{N}_{\mathbf{m}}^{\mathbf{e}} \bar{q}_{m} d \Gamma_{q_{m}}\right)
\end{aligned}
$$

and

$$
\begin{aligned}
\mathbf{K}_{\mathbf{f}} & =\Lambda_{e=1}^{n_{e l, f}}\left(\int_{\Omega_{f}} \mathbf{B}_{\mathbf{f}}^{\mathbf{e}^{\mathbf{T}}} \frac{h^{2}}{12 \mu} \mathbf{B}_{\mathbf{f}}^{\mathbf{e}} d \Omega_{f}\right) \\
\mathbf{Q}_{\mathbf{f}} & =\Lambda_{e=1}^{n_{e l, f}}-\left(\int_{\Gamma_{q_{f}}} \mathbf{N}_{\mathbf{f}}^{\mathbf{e}} \bar{q}_{f} d \Gamma_{q_{f}}\right)
\end{aligned}
$$




\section{Interaction between the FE meshes from porous medium and fractures}

This chapter presents the coupling technique used to describe the interaction between the finite element (FE) meshes from porous medium and fractures. The proposed technique is an extension of the approach developed by Bitencourt Jr et al. (2015) in the context of mechanical problems, in which the key concept to describe the interaction between non-matching meshes is based on the definition of the coupling finite elements (CFEs). Therefore, the CFEs are used in this research to enforce the continuity of the pressure field between the non-matching meshes.

An overview of the coupling procedure developed and the formulation of the CFE for hydraulic problems are described next.

\subsection{Overview of the procedure for coupling non-matching meshes}

The problem treated in this research is composed by a naturally fractured porous medium as illustrated in Figure 4.1(a). The discretization of the problem and the procedure adopted to couple the meshes from 2D porous medium and 1D discrete fractures is given by the following steps:

1. Discretization of the porous matrix (three-noded triangular elements) and fractures (two-noded bar elements) in finite elements in a totally independent way using the concept of overlapping non-matching meshes (Figure 4.1(b));

2. Generation of coupling finite elements (CFEs) combining nodes from fractures (red nodes) and porous matrix (Figure 4.1(c));

3. Assembly of the CFEs in the discrete system of equations of the hydraulic problem. 


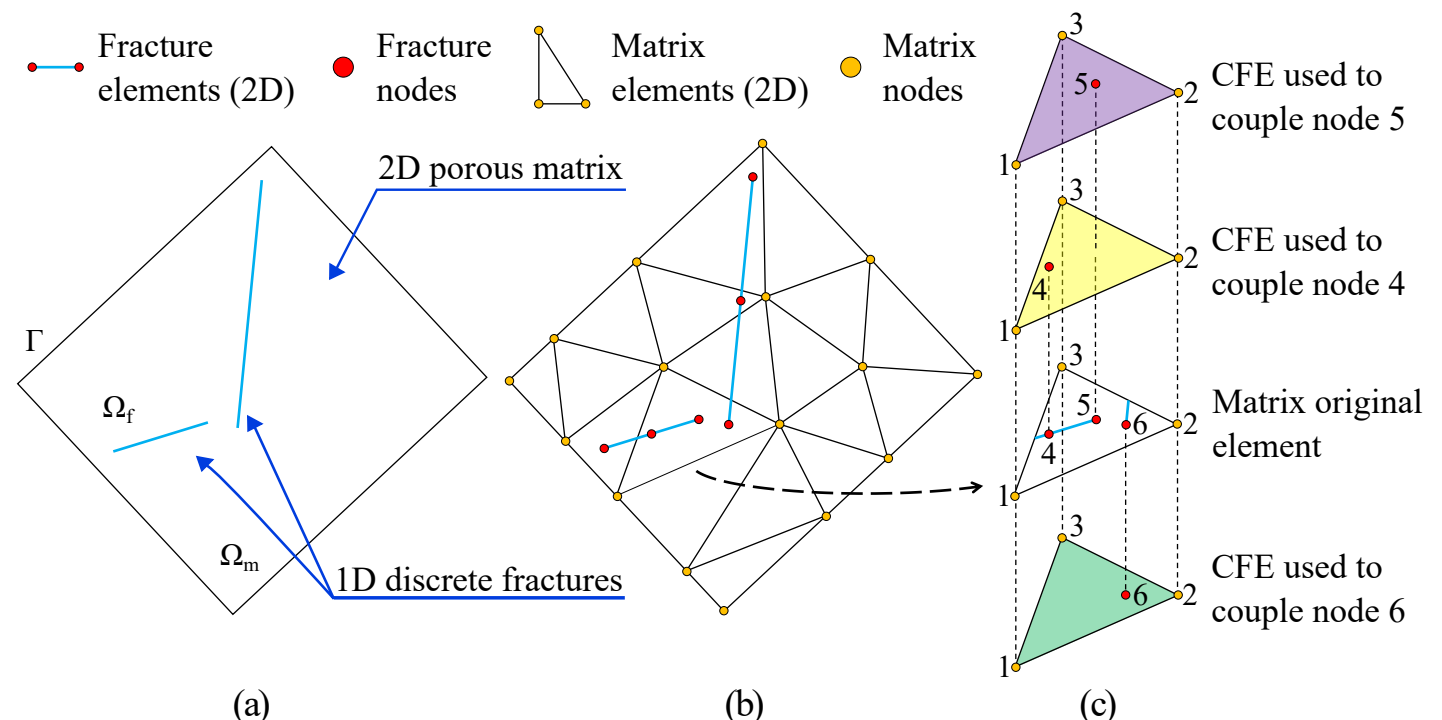

Figure 4.1: Schematic representation of the 2D coupling strategy for non-matching meshes: (a) porous matrix and fractures geometries; (b) mesh discretization of the porous matrix (three-noded triangular elements) and fracture (two-noded bar elements) domains; (c) Insertion of CFEs (four-noded triangular elements) to couple the meshes from porous medium and fracture.

As can be seen in Figure 4.1(c), each CFE has the same nodes of a matrix finite element and an extra node (coupling node), matching with the loose node that belongs to the fracture domain. As consequence, the CFEs overlap the standard finite element (Figure 4.1(b)) and each CFE is inserted using as base an existing finite element from porous matrix. In addition, it is important to note that for each loose node, one CFE is required.

Considering the example to couple the lose nodes 4, 5 and 6 depicted in Figure 4.1(c), three coupling finite elements are inserted: $C F E_{1}=\{1,2,3,4\}, C F E_{2}=\{1,2,3,5\}$, and $C F E_{3}=\{1,2,3,6\}$. In this example, the same finite element from porous matrix composed by the nodes 1,2 and 3 is used as the base element to create the CFEs.

After the introduction of the CFEs, these elements are used to enforce the continuity of the pressure field between the initially overlapping non-matching meshes from porous matrix and fractures. Therefore, considering the permeability matrices, vectors of pressures, and vectors of external fluxes developed separately for porous matrix and fracture domain in Section 3.2, the set of algebraic equations which describes the solution of the problem considering the coupling scheme for porous 
medium and fracture is given by:

$$
\underbrace{\left[\begin{array}{cc}
\mathbf{K}_{\mathbf{m}} & \mathbf{0} \\
\mathbf{0} & \mathbf{K}_{\mathbf{f}}
\end{array}\right]}_{\mathbf{K}} \underbrace{\left[\begin{array}{c}
\mathbf{P}_{\mathbf{m}} \\
\mathbf{P}_{\mathbf{f}}
\end{array}\right]}_{\mathbf{P}}=\underbrace{\left[\begin{array}{c}
\mathbf{Q}_{\mathrm{m}} \\
\mathbf{Q}_{\mathbf{f}}
\end{array}\right]}_{\mathbf{Q}}+\mathbf{Q}_{\mathbf{c}}
$$

where the vector $\mathbf{Q}_{\mathbf{c}}$ corresponds to the approximation of the fluid exchange between the matrix and each fracture. This vector can be written in terms of $\mathbf{P}_{\mathbf{m}}$ and $\mathbf{P}_{\mathbf{f}}$ by means of coupling finite elements and their permeability coupling matrix $\mathbf{K}_{\mathbf{c}}$ :

$$
\mathrm{Q}_{\mathrm{c}}=\mathbf{K}_{\mathrm{c}}\left[\begin{array}{c}
\mathbf{P}_{\mathrm{m}} \\
\mathbf{P}_{\mathrm{f}}
\end{array}\right]
$$

Finally, by substituting Eq. 4.2 into Eq.4.1, the discrete system of equations that describes the hydraulic problem can be written as:

$$
\left(\mathbf{K}-\mathbf{K}_{\mathbf{c}}\right) \mathbf{P}=\mathbf{Q}
$$

\subsection{Coupling finite element discretization}

Considering a three-noded triangular finite element of domain $\Omega_{m}^{e}$ used to discretize in $\mathrm{FE}$ the porous matrix. The pressure at any material point $(\mathbf{X}=(x, y))$ of its domain can be approximated, according to Eq. 3.6, as follows:

$$
p_{m}^{e}(x, y)=\sum_{i=1}^{3} N_{m(i)}(x, y) p_{m(i)}^{e}
$$

where the shape function $N_{m(i)}$ and nodal pressure $p_{m(i)}^{e}$ components are defined in Table 3.1.

The CFE shares nodes from the porous matrix and fracture. Thus, a CFE has the nodes of a three-noded triangular finite element and an additional node, called coupling node $\left(C_{\text {node }}\right)$, situated at the material point $\mathbf{X}_{\mathbf{c}}=\left(x_{c}, y_{c}\right) \in \Omega_{m}^{e}$, which corresponds to the loose node of the fracture on its domain (Figure 4.2).

Therefore, a relative pressure $\llbracket p \rrbracket$, can be defined for CFE as the difference between the pressure $p_{m}^{e}$ calculated at the material point $\left(x_{c}, y_{c}\right)$ using the Eq. 4.4 and the 


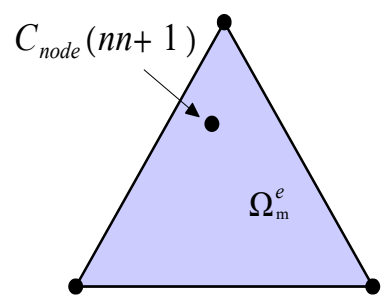

Figure 4.2: CFE - Four-noded triangular finite element.

pressure of the coupling node (the fourth node of the CFE), as follows:

$$
\llbracket p \rrbracket=p_{m}^{e}\left(x_{c}, y_{c}\right)-p_{C_{n o d e}(4)}^{e}=\sum_{i=1}^{3} N_{m(i)}\left(x_{c}, y_{c}\right) p_{m(i)}^{e}-p_{C_{n o d e}(4)}^{e}=\mathbf{B}_{\mathbf{c}}^{\mathbf{e}} \mathbf{p}_{\mathbf{c}}^{\mathbf{e}}
$$

where the matrix $\mathbf{B}_{\mathbf{c}}^{\mathbf{e}}$ is given by:

$$
\mathbf{B}_{\mathbf{c}}^{\mathbf{e}}=\left[\begin{array}{llll}
N_{m(1)}\left(x_{c}, y_{c}\right) & N_{m(2)}\left(x_{c}, y_{c}\right) & N_{m(3)}\left(x_{c}, y_{c}\right) & -1
\end{array}\right]
$$

The vector $\mathbf{p}_{\mathbf{c}}^{\mathbf{e}}$ which stores the pressure components of the CFE:

$$
\mathbf{p}_{\mathbf{c}}^{\mathbf{e}}=\left[\begin{array}{c}
p_{m(1)} \\
p_{m(2)} \\
p_{m(3)} \\
p_{C_{\text {node }}}^{e}(4)
\end{array}\right]
$$

\subsection{Fluid exchange vector and permeability matrix}

For hydraulic problems, an equivalent internal virtual work for the CFE can be obtained following an analogy between mechanical and hydraulic problems discussed in Segura and Carol (2004) and recently adopted by Damirchi et al. (2020):

$$
\delta W_{e}^{i n t}=\delta \llbracket p \rrbracket Q_{c}(\llbracket p \rrbracket)
$$

where $\delta \llbracket p \rrbracket$ is an arbitrary virtual relative pressure compatible with the boundary conditions of the problem; and $Q_{c}(\llbracket p \rrbracket)$ is the flux associated with the pressure drop at $C_{\text {node }}$. The virtual pressure drop is given by:

$$
\delta \llbracket p \rrbracket=\mathbf{B}_{\mathbf{c}}^{\mathbf{e}} \delta \mathbf{p}_{\mathbf{c}}^{\mathbf{e}}
$$


Thus, the fluid exchange between the matrix and each fracture of a CFE is obtained as

$$
\mathbf{Q}_{\mathbf{c}}^{\mathbf{e}}=\mathbf{B}_{\mathbf{c}}^{\mathbf{e}^{\mathbf{T}}} Q_{c}(\llbracket p \rrbracket)
$$

Assuming a linear relationship between the flux associated with the relative pressure and the relative pressure itself

$$
Q_{c}(\llbracket p \rrbracket)=C \llbracket p \rrbracket=C \mathbf{B}_{\mathbf{c}}^{\mathbf{e}} \mathbf{p}_{\mathbf{c}}^{\mathbf{e}}
$$

the Eq. 4.10 can be rewritten as:

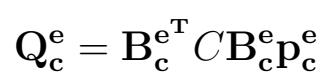

and the corresponding permeability matrix of the CFE can be obtained by:

$$
\mathbf{K}_{\mathbf{c}}^{\mathbf{e}}=\frac{\partial \mathbf{Q}_{\mathbf{c}}^{\mathbf{e}}}{\partial \mathbf{p}_{\mathbf{c}}^{\mathbf{e}}}=\mathbf{B}_{\mathbf{c}}^{\mathbf{e}^{\mathrm{T}}} C \mathbf{B}_{\mathbf{c}}^{\mathbf{e}}
$$

Finally, the global coupling permeability matrix can be obtained by a typical FE assemblage procedure of the form:

$$
\mathbf{K}_{\mathbf{c}}=\Lambda_{e=1}^{n_{e l, C F E}} \mathbf{K}_{\mathbf{c}}^{\mathbf{e}}
$$

Note that the interaction between the fracture mesh and porous matrix mesh is defined by the parameter $C$. Hence, $C=0$ leads to a situation in which the permeability of a mesh does not affect the pore pressure field of the other mesh and there is no flux exchange between them. Intermediate values of $C$ can also be used as leak-off parameter, but such cases are out of the scope of this work. In this research, a high value of $C$ is adopted (penalty parameter) to ensure the fully interaction between meshes (i.e., fracture and porous medium). 


\section{Numerical analyses}

In this chapter, the proposed embedded discrete fracture model for modeling fluid flow in naturally fractured reservoir is assessed. Three sets of numerical examples are performed involving several hydrodynamic scenarios with single and multiple fractures to assess the capability of the model to take into account some discrete fracture effects such as orientation, fracture-fracture intersection, and fracture-vug connectivity.

The porous matrix and fractures are discretized using three-noded elements and twonoded bar elements, respectively. In addition, structured and unstructured meshes with distinct mesh refinements are adopted in the discretization of the porous matrix to assess its influence on the pressure distribution. The capability of the coupling scheme to enforce the continuity of the pressure field between the non-matching meshes from porous matrix and fractures is carried out using four-noded triangular elements.

All the analyses are performed considering a steady-state flow regime, and the fluid is considered to be incompressible with a dynamic viscosity of $\mu=10^{-9}$ MPa.s. The fracture permeability is evaluated using the cubic law, as presented in Section 2.1.2. For the examples presented in Section 5.1.1, Section 5.1.2 and Section 5.2.2, the root mean square (RMS) was used to calculate the error between the result obtained via CFE method and a numerical reference curve, which can be written according to

$$
R M S=\sqrt{\frac{1}{N} \sum_{n=1}^{N}\left(p_{n, r e f}-p_{n, C F E}\right)^{2}}
$$

where $n=1,2, \ldots, N$ is the point in the curve, $N$ is the total number of points and $p_{n, r e f}$ and $p_{n, C F E}$ are the pressures measured in the reference curve and in that one 
resulting from the use of CFEs, respectively.

\subsection{Validation of the proposed model}

\subsubsection{Single vertical fracture}

This first problem consists of a vertical fracture with aperture of $5 \times 10^{-3} \mathrm{~m}$ located at $x=0.5$, and $y \in(0.2,0.8) m$ inside a square plane in the domain $\Omega(0,1)^{2}$. The geometry and boundary conditions are shown in Figure 5.1(a). Pressures of $p_{1}=1 \mathrm{MPa}$ and $p_{2}=2 \mathrm{MPa}$ are applied on the bottom left side $(0.1 \mathrm{~m})$ and upper right side $(0.1 \mathrm{~m})$ of the medium, respectively. The matrix permeability is $k_{m}=10^{-12} m^{2}(1013 m D)$.

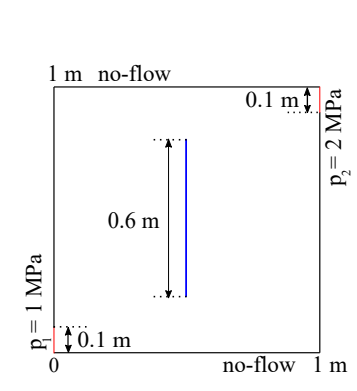

(a)

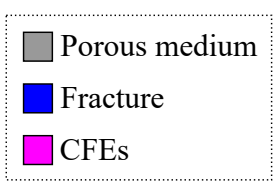

$$
\text { Mesh } 2
$$

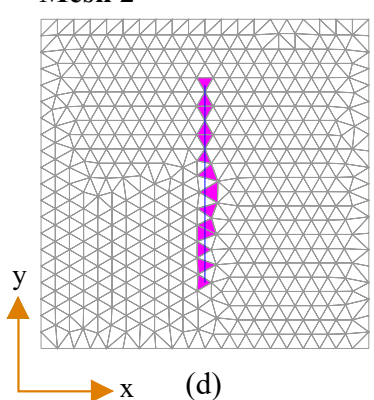

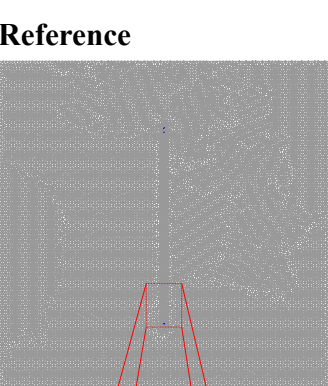

(b)

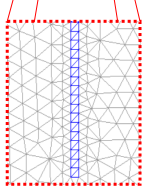

Mesh 3

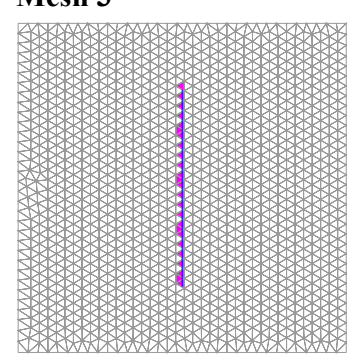

(e)

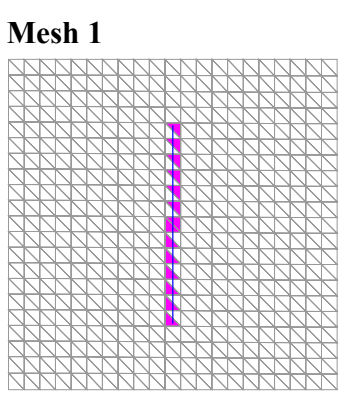

(c)

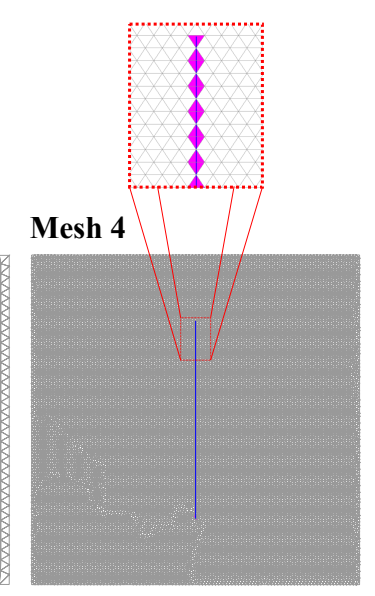

(f)

Figure 5.1: Vertical single fracture problem: (a) geometry and boundary conditions; (b) Reference mesh; (c) Mesh 1 (structured mesh); (d) Mesh 2 (unstructured and coarse mesh); (e) Mesh 3 (unstructured and intermediate mesh) and (f) Mesh 4 (unstructured and fine mesh).

Figure 5.1(b) illustrates the equidimensional and matching mesh used as Reference solution. Equidimensional mesh means that both the fracture and the porous 
medium are discretized in $2 \mathrm{D}$, and matching mesh means that the nodes at the interfaces between the porous medium and fracture are shared by both domains. For this Reference mesh, a highly refined mesh is adopted. For the analyses using the proposed approach, four non-matching meshes were developed. Mesh 1 is discretized using structured grid (Figure 5.1(c)), and Meshes 2, 3 and 4 are discretized using unstructured grids and levels of refinement represented by coarse, intermediate and fine meshes, respectively (Figure 5.1(d), (e) and (f)). Table 5.1 presents the features of the meshes employed in the numerical simulations. It is interesting to note that the number of CFEs is a function of the fracture discretization. For these analyses, a coupling parameter $C=1.0 \times 10^{-2} \mathrm{~m} /($ MPa.s $)$ was adopted.

Table 5.1: Features of the FE meshes employed for the numerical simulations of the single vertical fracture problem.

\begin{tabular}{|c|c|c|c|c|c|c|}
\hline \multirow{2}{*}{ Case } & \multirow{2}{*}{ DOF } & \multicolumn{2}{|c|}{ Porous medium } & \multicolumn{2}{c|}{ Fracture } & \multirow{2}{*}{ CFEs } \\
\cline { 3 - 6 } & & Elements & Size (m) & Elements & Size (m) & \\
\hline Reference & 11884 & 23126 & $0.010 \longrightarrow 0.005$ & 480 & 0.005 & - \\
\hline Mesh 1 & 498 & 882 & 0.047 & 13 & 0.046 & 14 \\
\hline Mesh 2 & 496 & 882 & 0.050 & 13 & 0.046 & 14 \\
\hline Mesh 3 & 1341 & 2498 & 0.030 & 24 & 0.025 & 25 \\
\hline Mesh 4 & 11679 & 22804 & 0.010 & 75 & 0.008 & 76 \\
\hline
\end{tabular}

The pressure distribution obtained for the Reference solution and for the four cases using the coupling procedure (for Meshes 1 to 4) are presented in Figure 5.2. As the fracture permeability is higher than the one of the porous medium, a preferential flow path is given by the fracture domain.

Figure 5.3 illustrates three pressure profiles plotted along horizontal lines located at $y_{1}=0 \mathrm{~m}$ that corresponds to the bottom face of domain, at $y_{2}=0.2 \mathrm{~m}$ that corresponds to the line that crosses the bottom endpoint of the fracture, and at $y_{3}=0.5 \mathrm{~m}$ that corresponds to the line that crosses the middle point of the fracture. Furthermore, the numerical results obtained are compared against those obtained by Zeng et al. (2020) using a Discrete Fracture Model (DFM) and Phase Field based Discrete Fracture Model (PFDFM) to solve this same problem.

For $y_{1}=0 m$ the pressure distribution presents a continuum behavior, once there is no interference of the fracture in the pressure profile (Figure 5.3(a)). For the pressure profile along $y_{2}=0.2 \mathrm{~m}$, as can be seen in Figure 5.3(b), the pressure distribution increases until it crosses the fracture tip, where the pressure is around 1.5 MPa due to the symmetry of the geometry and boundary conditions. A similar 


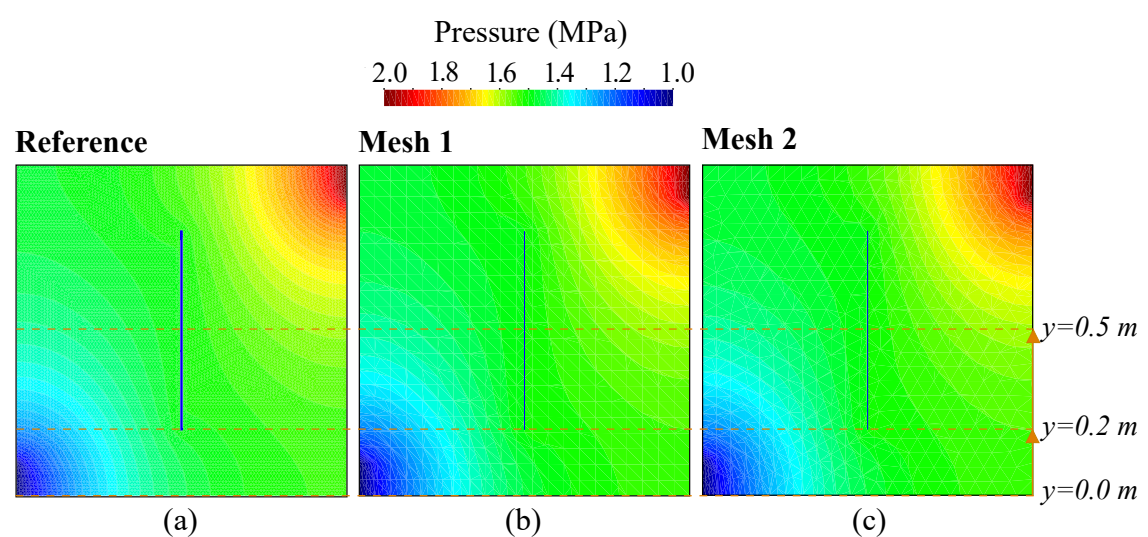

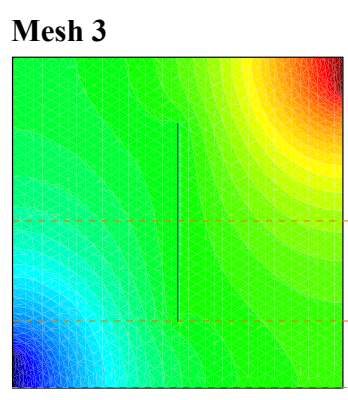

(d)
Mesh 4

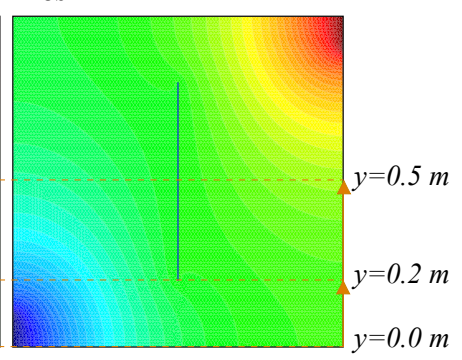

(e)

Figure 5.2: Pressure distribution of the single vertical fracture problem: (a) Reference; (b) Mesh 1; (c) Mesh 2; (d) Mesh 3; and (e) Mesh 4.

behavior occurs for pressure profile at $y_{3}=0.5 \mathrm{~m}$ presented in Figure 5.3(c), but this curve is smoother than the previous one. Note that results obtained using the proposed scheme, independent of the mesh type (i.e., structured and unstructured) have good agreement with Reference solution and the results obtained by Zeng et al. (2020). In addition, the pressure fields obtained show a continuum behavior between fracture and porous medium. For all cases with unstructured meshes, the refinement of $\mathrm{FE}$ meshes was able to treat the divergence of the pressure distribution results close to the boundary conditions applied.

To investigate the influence of the values adopted for the penalty parameter on the pressure field, additional numerical analyses varying $C$ from $10^{-10}$ to $10^{-2} \mathrm{~m} /(\mathrm{MPa.s})$ are performed. The analyses are carried for the four non-matching meshes, and an error is evaluated based on the root mean square (Eq. 5.1) with respect to the Reference mesh for the points in the pressure profile curve at $y_{2}=0.2 \mathrm{~m}$. Based on the results illustrated in Figure 5.4, it can be noted a clear convergence for values between $10^{-4}$ and $10^{-2} \mathrm{~m} /(\mathrm{MPa}$.s $)$. For lower values, numerical instability (i.e. ill-conditioning of the system matrices) can be observed. However, this drawback 


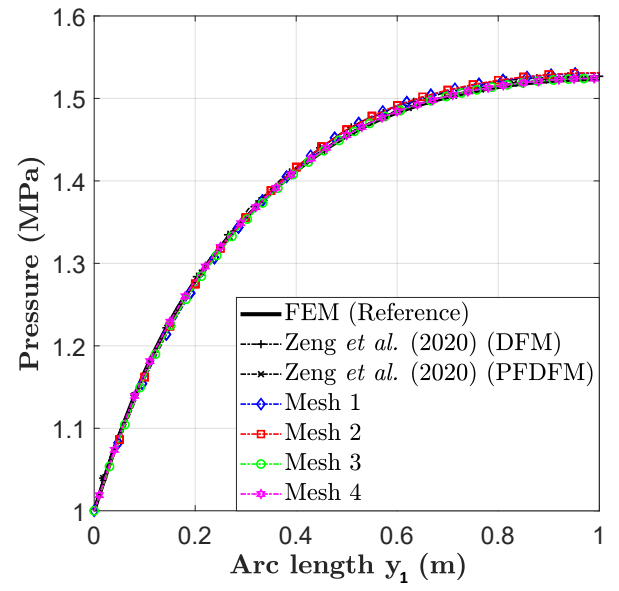

(a)

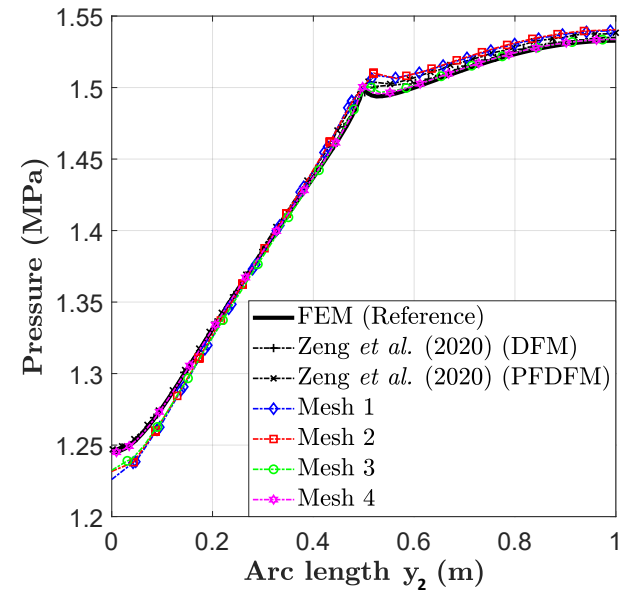

(b)

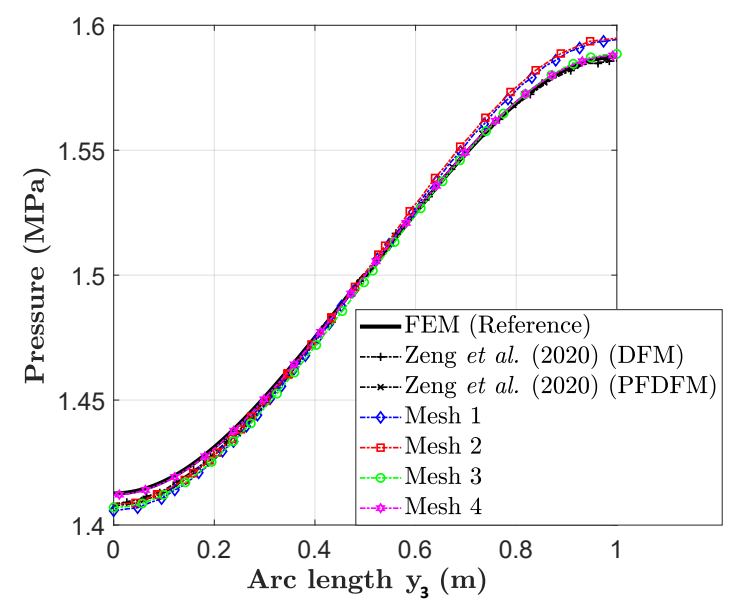

(c)

Figure 5.3: Pressure profile of the single vertical fracture problem at: (a) $y_{1}=0 \mathrm{~m}$; (b) $y_{2}=0.2 \mathrm{~m}$; and (c) $y_{3}=0.5 \mathrm{~m}$.

can be overcome by using suitable values and, therefore, for all the next examples studied in this research, $C=10^{-2} \mathrm{~m} /(\mathrm{MPa}$.s $)$ is adopted. 


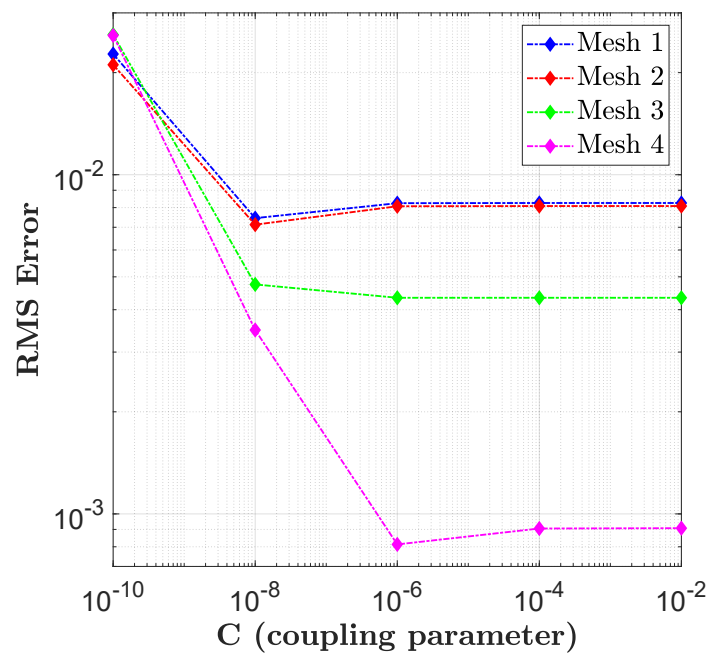

Figure 5.4: RMS error curves for the pressure profile crossing at $y_{2}=0.2 \mathrm{~m}$.

\subsubsection{Single diagonal fracture}

This second problem is composed by a single diagonal fracture with aperture of $5 \times 10^{-3} \mathrm{~m}$ and $0.6 \mathrm{~m}$ of length inside the porous medium of domain $\Omega(0,1)^{2}$. Five case studies are considered with different fracture orientations that ranges from $30^{\circ}$ to $135^{\circ}$. Figure 5.5(a) illustrates the geometry of the problem and the boundary conditions applied. Pressures of $p_{1}=1 M P a$ and $p_{2}=2 M P a$ are applied in bottom left side $(0.1 \mathrm{~m})$ and upper right side $(0.1 \mathrm{~m})$ of the domain, respectively. The permeability of porous medium adopted is $k_{m}=10^{-12} \mathrm{~m}^{2}(1013 \mathrm{mD})$. Figure 5.5 illustrates the five case studies composed of fractures oriented $30^{\circ}$ (Figure $5.5(\mathrm{a})$ ), $45^{\mathrm{o}}$ (Figure 5.5(b)), 60 (Figure 5.5(c)), 90ㅇ (Figure 5.5(d)), and 135ㅇ (Figure 5.5(e)) from vertical axis (clockwise). For all the cases, the length and aperture of the fracture are constants. The five cases were studied including three types of mesh discretization. The first is an equidimensional and conforming mesh, i.e., the fracture domain and porous medium domain are discretized using three-noded triangular finite elements and the nodes at interfaces between the fracture and porous medium are shared by both domains, as illustrated in Figure 5.6.

The second type of mesh discretization is also performed using conforming meshes. However, in this case study, the porous medium is represented by three-noded triangular finite elements, while the fracture is represented by two-noded bar finite elements, as shown in Figure 5.7. 


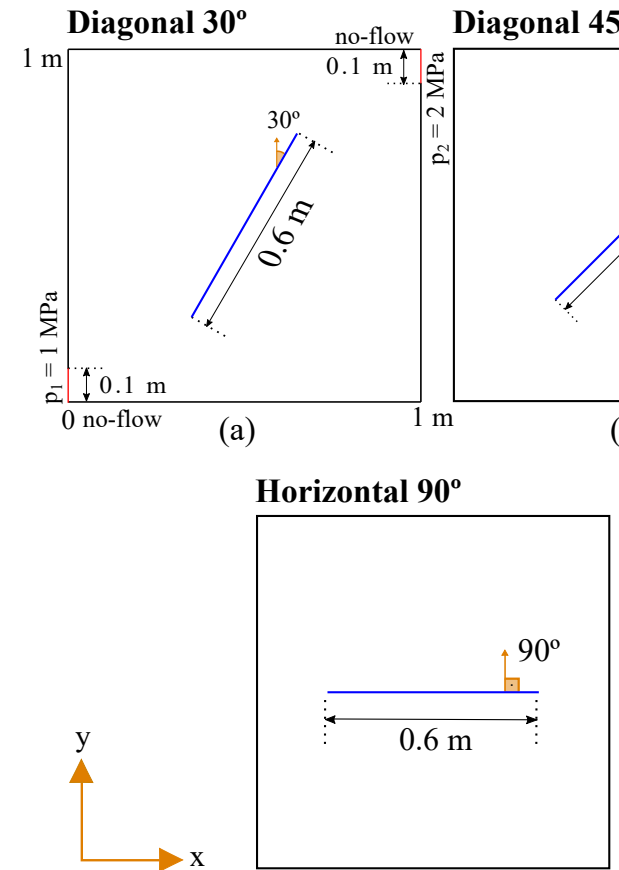

(d)

\section{Diagonal $60^{\circ}$}

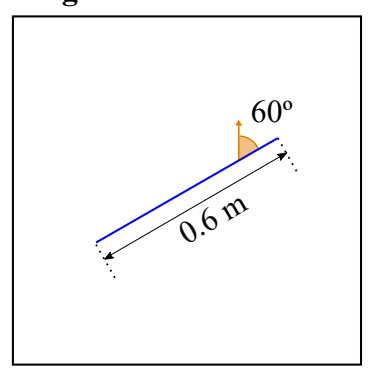

(c)

(b)

Diagonal $135^{\circ}$

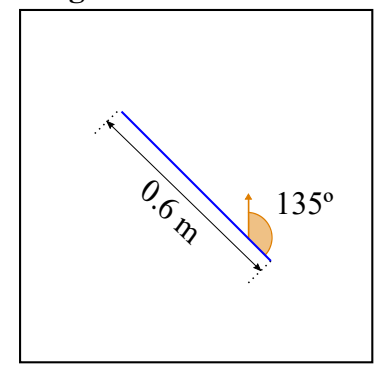

(e)

Figure 5.5: Single diagonal fracture problem: (a) boundary conditions applied and

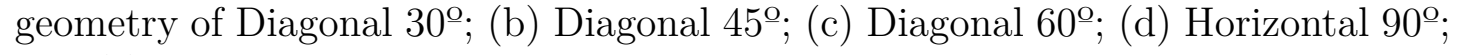
and (e) Diagonal $135^{\circ}$.

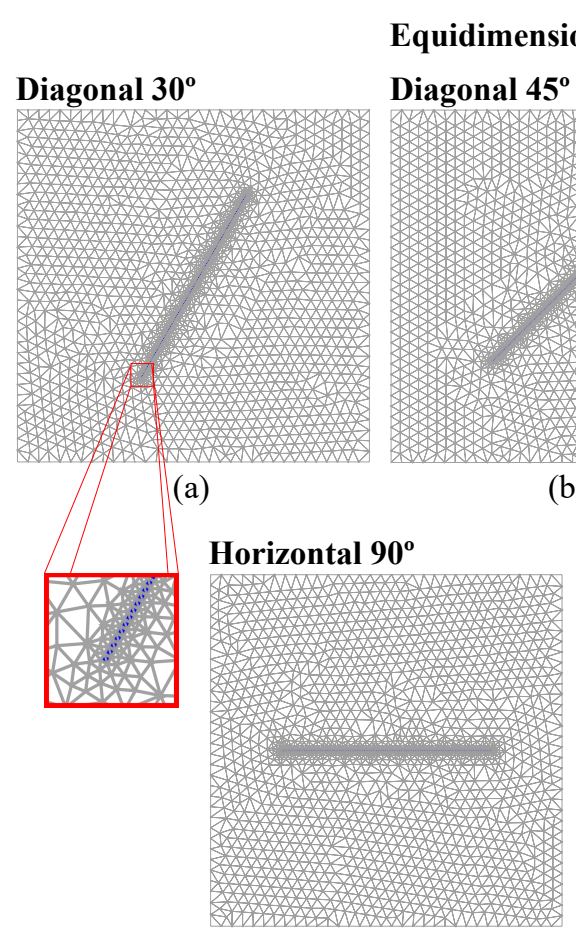

(d)

(b)

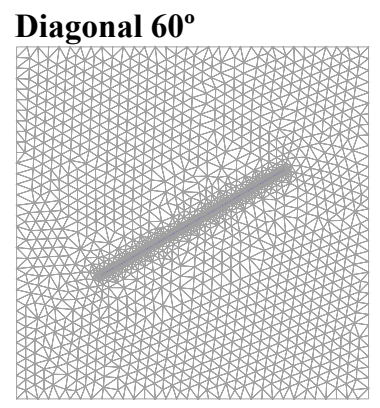

(c)

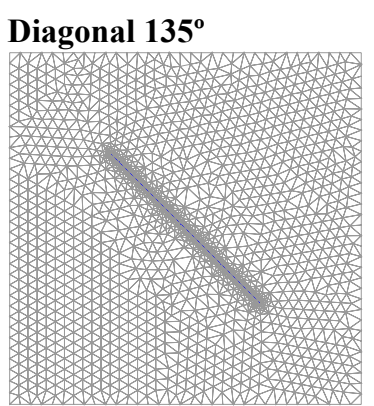

(e)

Figure 5.6: Equidimensional and conforming meshes used in the numerical analyses of the single diagonal fracture problem: (a) Diagonal 30\% (c) Diagonal 60\% ; (d) Horizontal 90\% ; and (e) Diagonal 135º . 


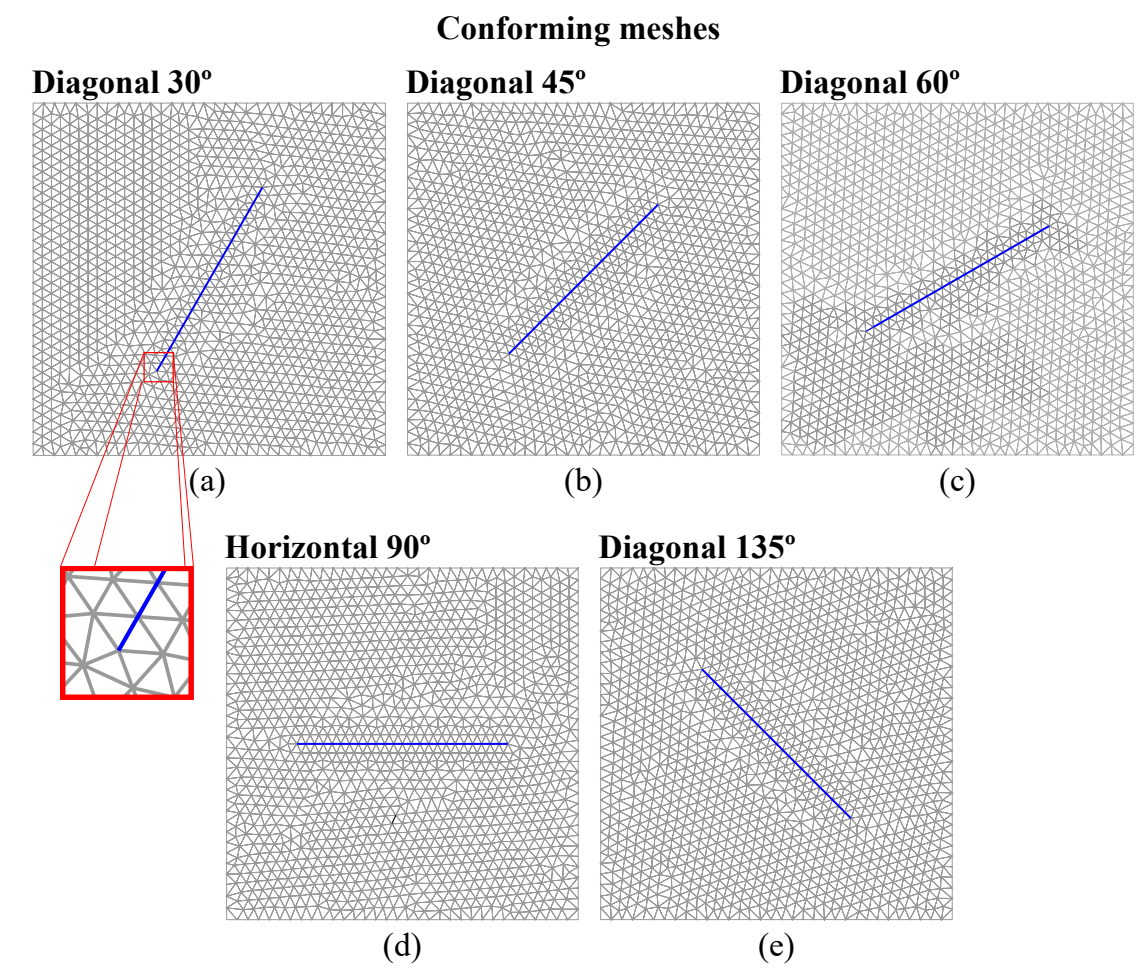

Figure 5.7: Conforming meshes used in the numerical analysis of the single diag-

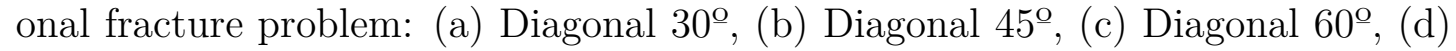
Horizontal $90^{\circ}$ and (e) Diagonal $135^{\circ}$.

In the third case study, non-matching meshes is adopted, i.e., the fracture and porous media are initially discretized independently, and then, CFEs are introduced to couple the meshes from the distinct domains, as illustrated in Figure 5.8.

Table 5.2 presents the main characteristics of the finite element meshes employed for three case studies.

The results obtained in terms of pressure distribution for the three case studies of the diagonal fracture problem are illustrated in Figure 5.9. As can be seen in this figure, the results obtained with the proposed approach are in good agreement with those obtained with Equidimensional and Conforming meshes. 


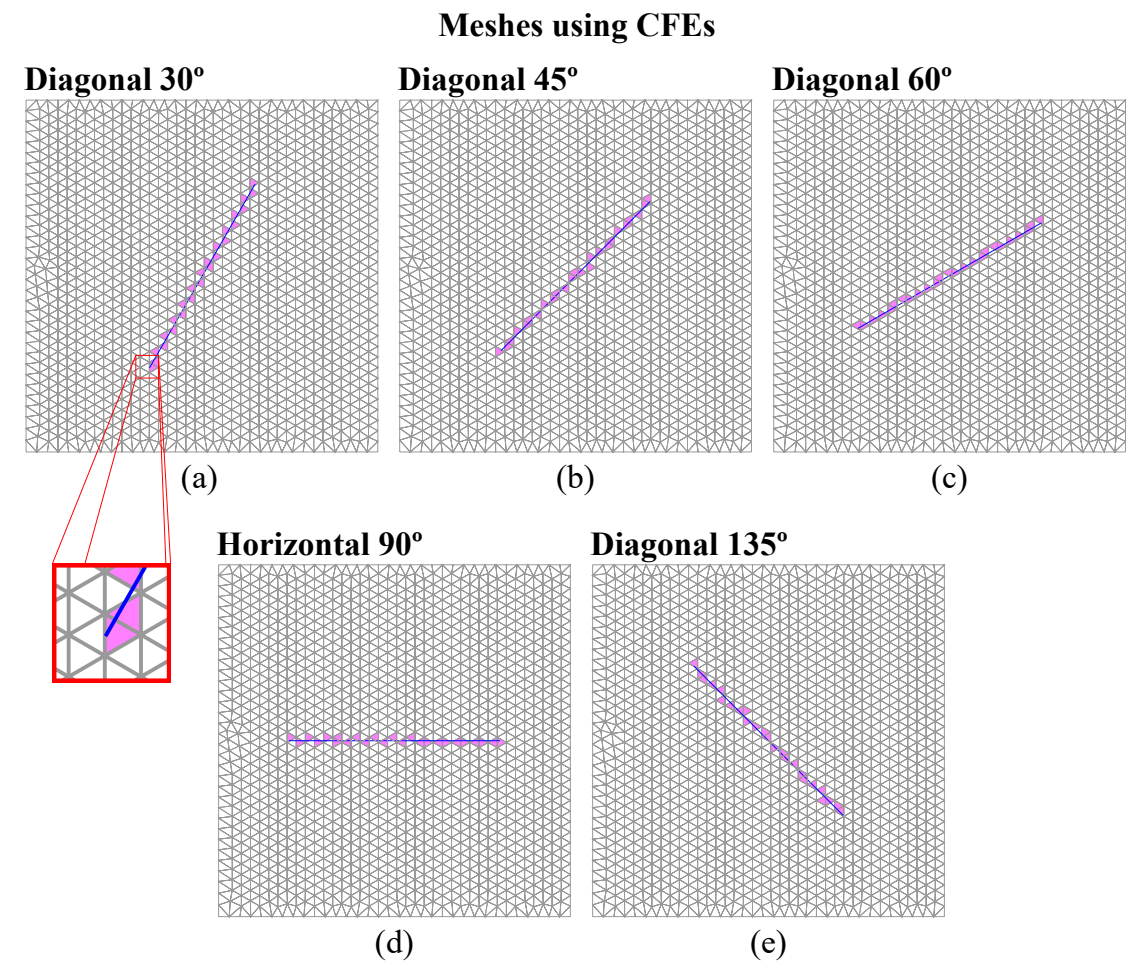

Figure 5.8: Meshes with CFEs in the numerical analysis of the single diagonal fracture problem: (a) Diagonal 30; (b) Diagonal 45; ; (c) Diagonal 60% (d) Horizontal $90^{\circ}$; and (e) Diagonal $135^{\circ}$.

For a better comprehension of the influence of the fracture inclinations, the pressure profiles for the three case studies were plotted along $y=0.5 \mathrm{~m}$. As can be seen in Figure 5.10, the model was able to capture the influence of fracture inclination on the pressure distribution of the porous matrix. For the cases Diagonal $30^{\circ}$ (Figure 5.10(a)) and Diagonal $45^{\circ}$ (Figure 5.10(a)), where the fractures are approximately aligned with the essential boundary conditions, the curves present a continuum behavior between fracture and matrix domains. Similar behavior occurs for the Diagonal $135^{\circ}$, but this curve is smoother than the previous ones, once the fracture is approximately perpendicular to the essential boundary conditions. For the cases where the fractures tend to be horizontal (From Diagonal $60^{\circ}$ to Horizontal $90^{\circ}$ ) the pressure distribution increases until it crosses the fracture and remains constant the more horizontally the fracture is aligned. 
Table 5.2: Characteristics of the FE meshes employed for the numerical simulations of the single diagonal fracture problem.

\begin{tabular}{|c|c|c|c|c|c|c|}
\hline \multirow{2}{*}{ Case } & \multirow{2}{*}{ DOF } & \multicolumn{2}{|c|}{ Porous medium } & \multicolumn{2}{|c|}{ Fracture } & \multirow{2}{*}{ CFEs } \\
\hline & & Elements & Size $(\mathbf{m})$ & Elements & Size $(\mathbf{m})$ & \\
\hline \multicolumn{7}{|c|}{ Equidimensional meshes } \\
\hline Diagonal $30^{\mathrm{O}}$ & 2103 & 3592 & \multirow{5}{*}{$0.005 \longrightarrow 0.03$} & \multirow{5}{*}{480} & \multirow{5}{*}{0.005} & \multirow{5}{*}{-} \\
\hline Diagonal $45^{-}$ & 2067 & 3520 & & & & \\
\hline Diagonal $60^{\circ}$ & 2091 & 3568 & & & & \\
\hline Horizontal $90^{\circ}$ & 2087 & 3560 & & & & \\
\hline Diagonal $135^{\circ}$ & 2079 & 3544 & & & & \\
\hline \multicolumn{7}{|c|}{ Conforming meshes } \\
\hline Diagonal $30^{\mathrm{O}}$ & 1311 & 2488 & \multirow{5}{*}{0.03} & \multirow{5}{*}{24} & \multirow{5}{*}{0.025} & \multirow{5}{*}{-} \\
\hline Diagonal $45^{\mathrm{O}}$ & 1314 & 2494 & & & & \\
\hline Diagonal $60^{\circ}$ & 1298 & 2462 & & & & \\
\hline Horizontal $90^{\circ}$ & 1314 & 2494 & & & & \\
\hline Diagonal $135^{\circ}$ & 1321 & 2508 & & & & \\
\hline \multicolumn{7}{|c|}{ Meshes using CFEs } \\
\hline Diagonal $30^{\mathrm{O}}$ & \multirow{5}{*}{1341} & \multirow{5}{*}{2498} & \multirow{5}{*}{0.03} & \multirow{5}{*}{24} & \multirow{5}{*}{0.025} & \multirow{5}{*}{25} \\
\hline Diagonal $45^{\mathrm{O}}$ & & & & & & \\
\hline Diagonal $60^{\circ}$ & & & & & & \\
\hline Horizontal $90^{\circ}$ & & & & & & \\
\hline Diagonal $135^{\circ}$ & & & & & & \\
\hline
\end{tabular}


Pressure $(\mathrm{MPa})$

$\begin{array}{lllllll}2.0 & 1.8 & 1.6 & 1.4 & 1.2 & 1.0\end{array}$

Equidimensional mesh

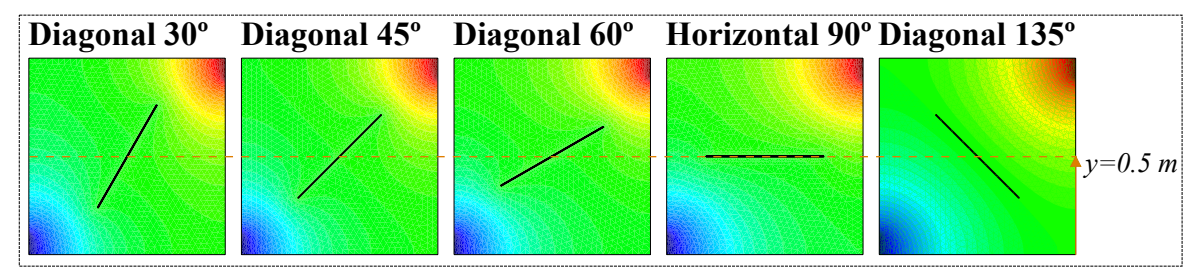

(a)

Conforming mesh

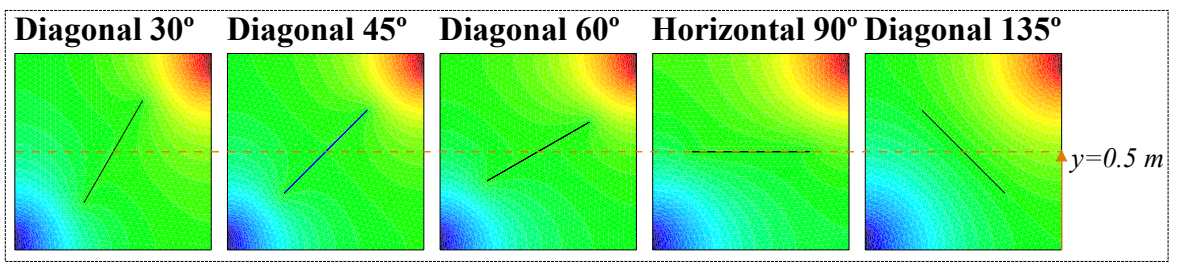

(b)

CFEs mesh

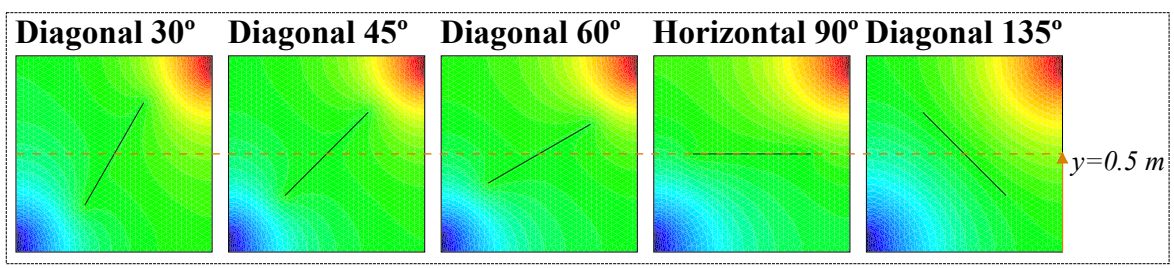

(c)

Figure 5.9: Pressure field for the single diagonal fracture problem: (a) Equidimensional mesh; (b) Conforming mesh; and (c) CFEs mesh. 


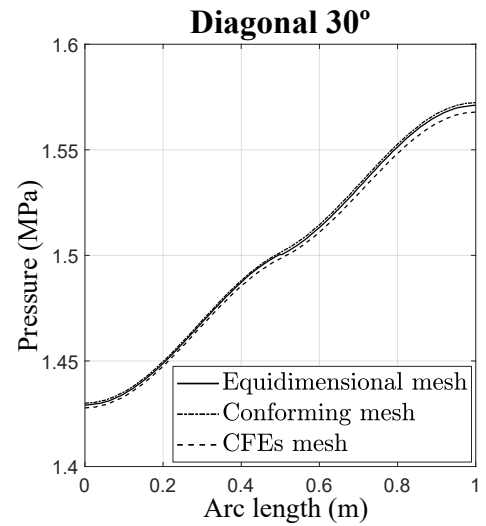

(a)

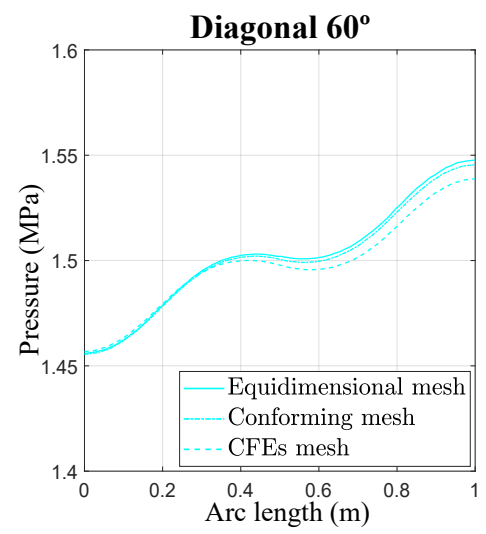

(c)

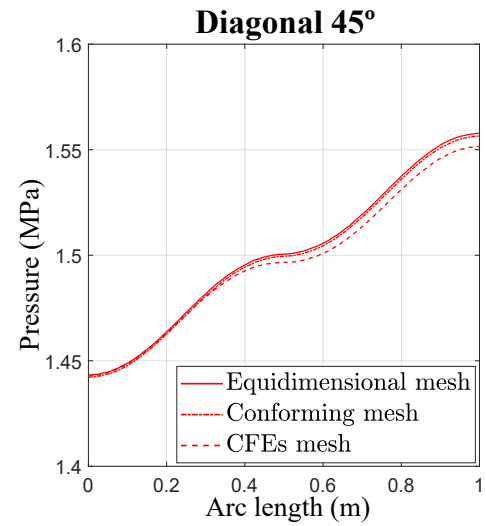

(b)

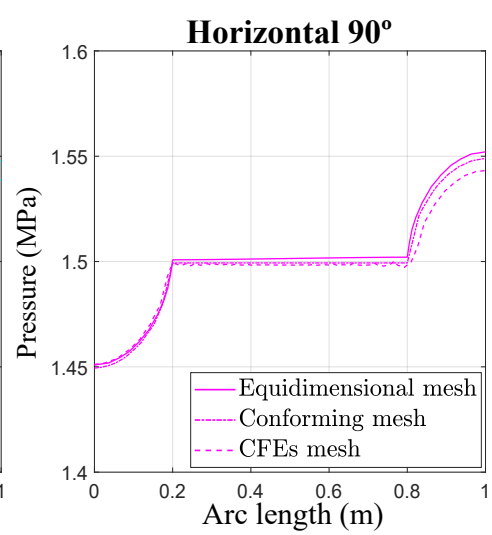

(d)

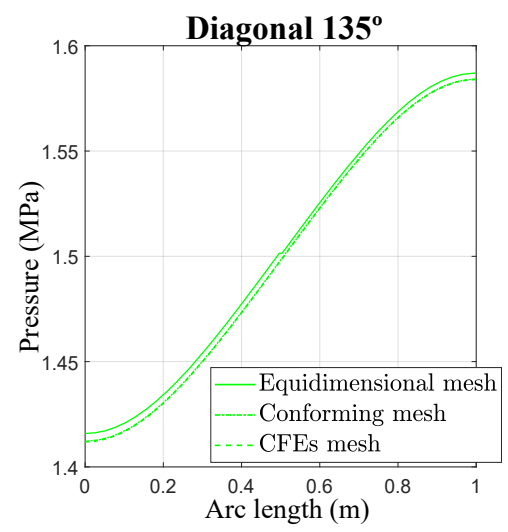

(e)

Figure 5.10: Pressure profile for the three case studies of the single diagonal fracture problem along $y=0.5 \mathrm{~m}$ : (a) Diagonal 30º; (b) Diagonal 45º; (c) Diagonal

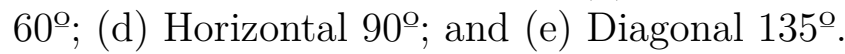

The pressure profile along $y=0.5 \mathrm{~m}$ for the $15 \mathrm{FE}$ meshes listed in Table 5.2 are plotted in Figure 5.11. As can be seen in this figure, the results obtained using CFEs presents good concordance with those obtained with equidimensional and conforming meshes. To assess the performance of the coupling procedure, the RMS 
error was calculated (Eq. 5.1) with respect to equidimensional meshes (EQ/CFE) and conforming meshes $(\mathrm{CF} / \mathrm{CFE})$. Based on the results presented in Table 5.3 the RMS is satisfactory for both comparisons.

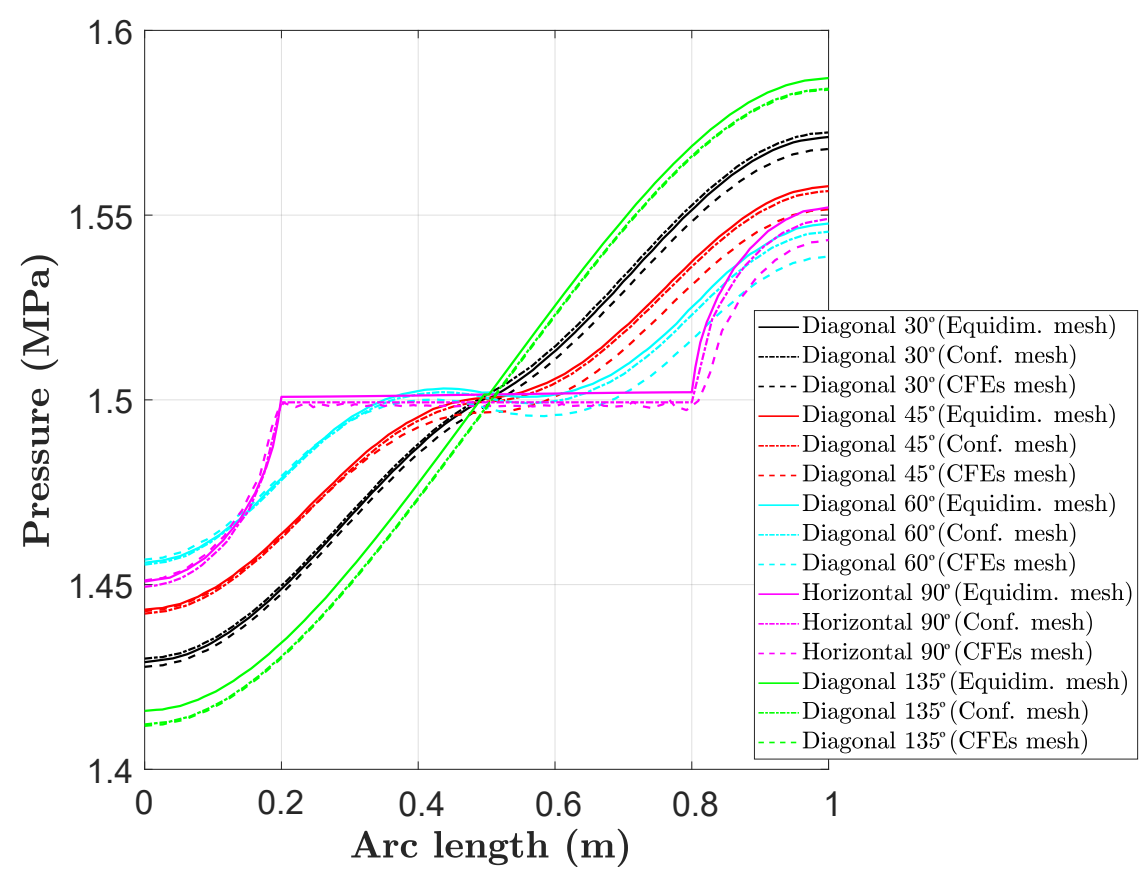

Figure 5.11: Pressure profile for the 15 meshes considered in the numerical analyses of the single diagonal fracture problem at $y=0.5 \mathrm{~m}$.

Table 5.3: RMS error values for the 5 cases studied.

\begin{tabular}{|c|c|c|}
\hline Case & EQ/CFE & CF/CFE \\
\hline Diagonal 30 & $2,26 \times 10^{-3}$ & $3,33 \times 10^{-3}$ \\
\hline Diagonal 45 & $4,26 \times 10^{-3}$ & $3,28 \times 10^{-3}$ \\
\hline Diagonal 60 & $1,47 \times 10^{-3}$ & $4,16 \times 10^{-3}$ \\
\hline Horizontal 90 & $7,62 \times 10^{-3}$ & $3,46 \times 10^{-3}$ \\
\hline Diagonal 135 & $3,61 \times 10^{-3}$ & $3,54 \times 10^{-4}$ \\
\hline
\end{tabular}

\subsection{Mesh refinement study}

This section presents two numerical examples to study the effect of mesh refinement. In the first case, a porous medium with two intersected fractures is studied using six different discretizations, and the numerical solutions obtained are compared with Numerical Mainfold Method proposed by $\mathrm{Hu}$ et al., 2016. The second case consists 
of a regular fracture network embedded in the porous medium. For this case, twelve examples are simulated using three types of mesh discretization and the numerical results are also compared with methods studied previously by Flemisch et al., 2018.

\subsubsection{Intersected fractures}

This example is formed by two inclined fractures intersecting each other on the center of the square plane of domain $\Omega(0,2)^{2}$ and are located at $y=-1+2 x, x$ $\in(0.5,1.5)$ and $y=3-2 x, x \in(0.5,1.5)$, respectively. Both fractures have an aperture of $1 \times 10^{-2} \mathrm{~m}$. Figure 5.12 (a) presents the geometry of the problem and the boundary conditions. As illustrated in this figure, no-flow Neumann conditions are applied on the top and bottom sides, and Dirichlet conditions are applied on the right and left sides of $p_{1}=0.1 \mathrm{MPa}$ and $p_{2}=0$, respectively. The matrix permeability adopted is $k_{m}=10^{-12} m^{2}(1013 \mathrm{mD})$.

Six different mesh discretization are considered. For all the cases the fractures are discretized as 1D discrete elements. The Reference mesh consists of matching (or conforming) meshes from porous matrix and fractures, as illustrated in Figure 5.12(a). For the other five cases (from Figure 5.12(d) to Figure 5.12(h)) overlapped non-matching meshes are considered. For all these cases the discretization of the porous medium remains the same. In addition, to study the influence of the presence of fracture node on the fracture intersection, three cases are considered: matching fracture with intersection node (i.e. the fractures share the same node at the fracture intersection) (Figure 5.12(d)); fracture overlapped with intersection node (i.e the fractures have initially independent nodes at the position of the fracture intersection) (Figure 5.12(d)); and no intersection node (i.e. there is no node at the fracture intersection) (Figure 5.12(d)). These three cases are simulated using a fracture mesh called coarse mesh. Finally, two additional cases are considered to study the influence of the fracture mesh refinement for the no intersection cases. Figure 5.12(g) and Figure 5.12(h) illustrate the intermediate and refined meshes adopted for these two cases. 


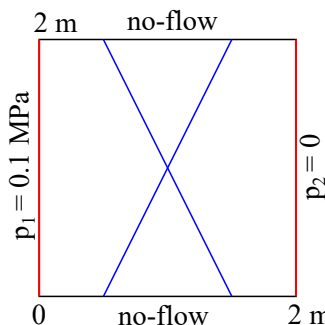

(a)

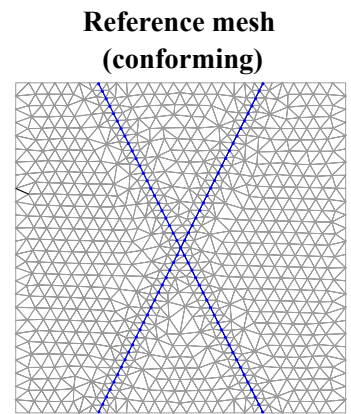

(c)

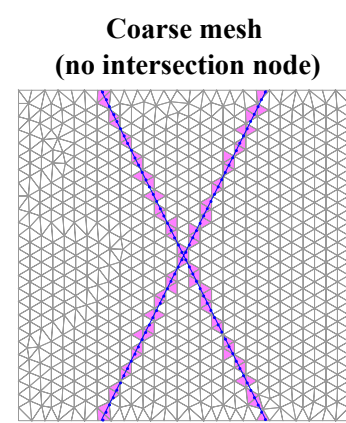

(f)

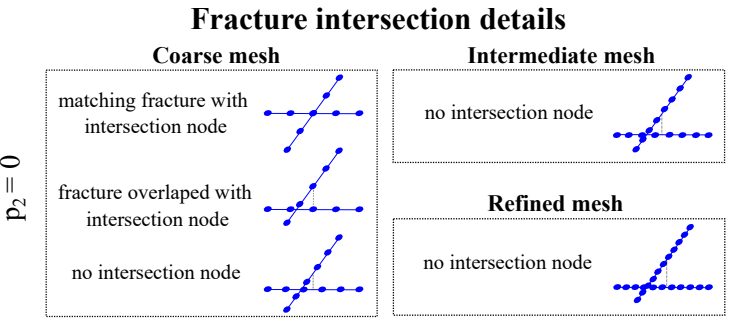

(b)

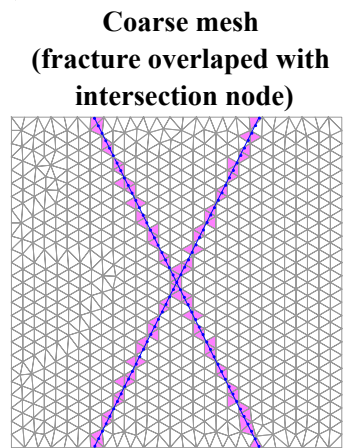

(e) (matching fracture with intersection node)

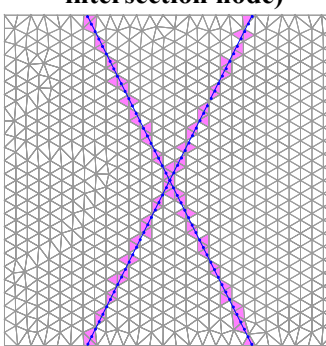

(d)

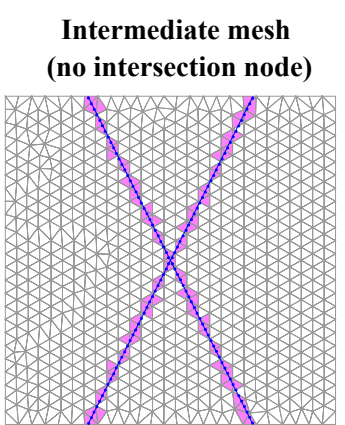

(g)

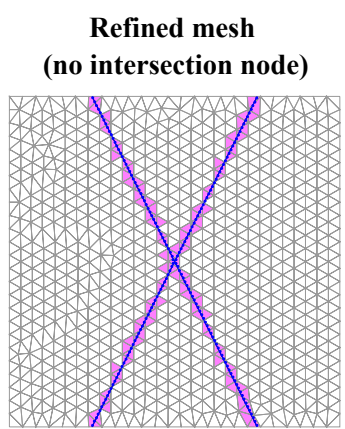

(h)

Figure 5.12: Intersected fractures problem: (a) geometry and boundary conditions; (b) details of fracture intersection for each mesh; (c) Reference mesh; (d) Coarse mesh (matching fractures with intersection node); (e) Coarse mesh (non-matching fractures with overlapped intersection node); (e) Coarse mesh (non-matching fractures with no intersection node); (f) Intermediate mesh (nonmatching fractures with no intersection node); and (h) Refined mesh (nonmatching fractures with no intersection node).

Further properties of the mesh discretization are listed in Table 5.4. 
Table 5.4: Characteristics of the FE meshes employed for the numerical simulations of the intersected fracture problem.

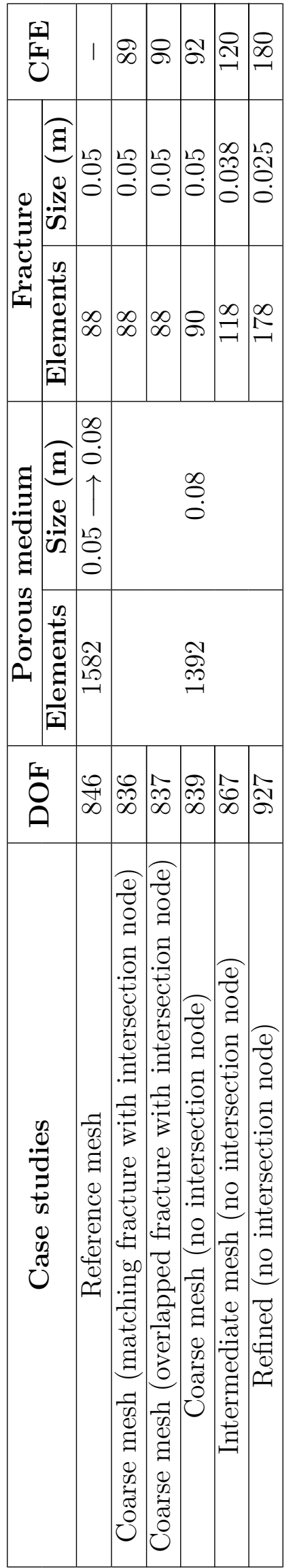


The pressure distribution obtained for all cases are illustrated in Figure 5.13 and present a good agreement between all the cases studied. Figure 5.14 illustrates the pressure profile crossing the intersection point of fractures at $y=1 \mathrm{~m}$. As can be observed in this figure, the five non-matching meshes results are similar and are in good agreement with the Reference solution. Moreover, considering the Reference solution, the results obtained with the proposed approach show better precision when compared to numerical approach proposed by $\mathrm{Hu}$ et al. (2016). Finally, the presence of fracture node on the fracture intersection and the mesh refinement do not improve substantially the accuracy of the results.

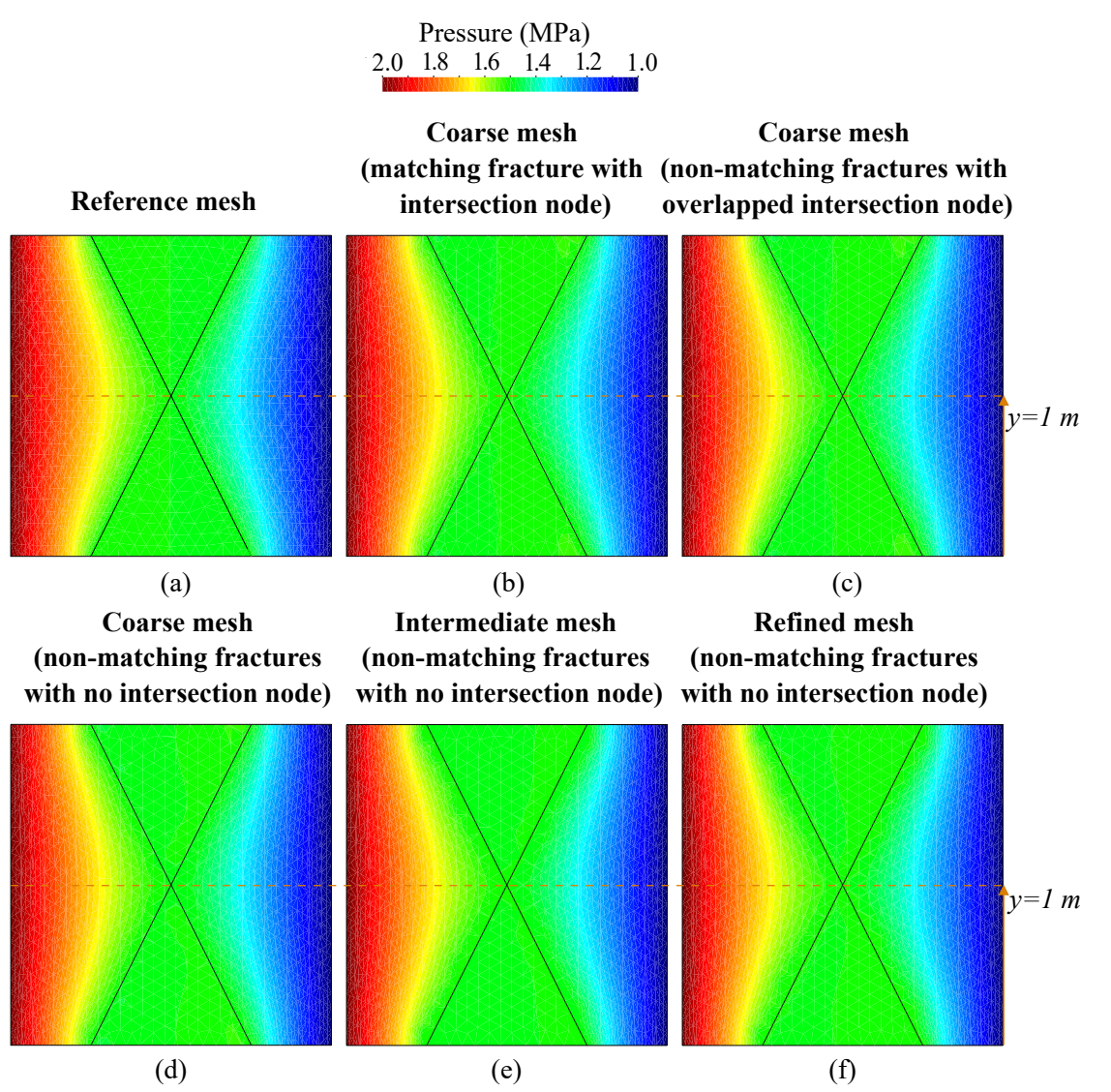

Figure 5.13: Pressure gradient of the intersected fractures problem: (a) Reference mesh; (b) Coarse mesh (matching fractures with intersection node); (c) Coarse mesh (non-matching fractures with overlapped intersection node); (d) Coarse mesh (non-matching fractures with no intersection node); (e) Intermediate mesh (non-matching fractures with no intersection node); and (f) Refined mesh (nonmatching fractures with no intersection node). 


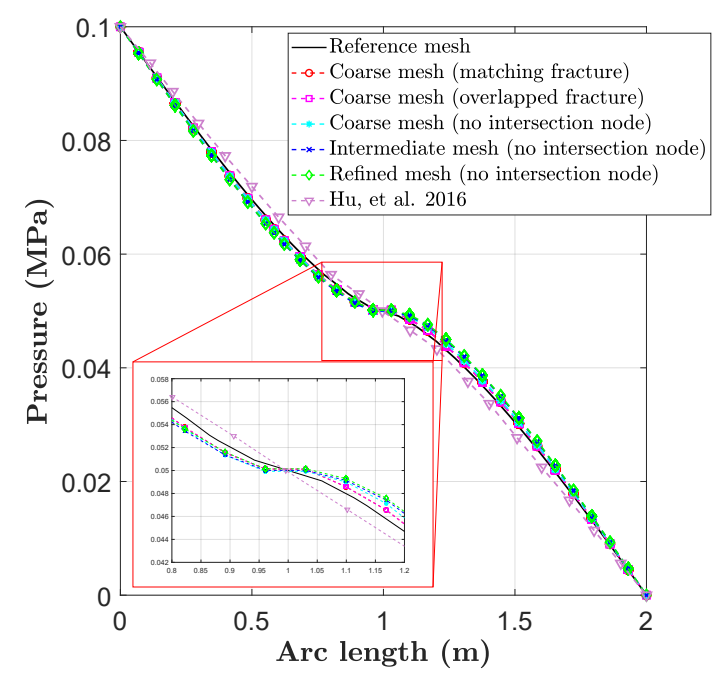

Figure 5.14: Pressure profile crossing the intersection of fractures at $y=1 \mathrm{~m}$ of the intersected fracture problem.

\subsubsection{Regular fracture network}

This example was firstly proposed by Geiger et al. (2013) and then improved to be used as a benchmark by Flemisch et al. (2018). The problem is formed by a regular fracture network with constant aperture of $1 \times 10^{-4} \mathrm{~m}$ embedded in the unit square plane $\Omega=(0,1)^{2}$. Top and bottom boundary faces have homogeneous Neumann conditions of no-flow $(q=0.0)$, the left boundary face has a constant fluid flow of $q=-1 \mathrm{~m} / \mathrm{s}$, and the right boundary face has Dirichlet condition of $p=1 M P a$, as shown in Figure 5.15. This same figure also presents two cut lines $(x=0.5 \mathrm{~m}$ and $y=0.7 \mathrm{~m}$ ), in which Flemisch et al. (2018) measured the pressure field and compared the responses. The rock and fracture permeabilities are $k_{m}=10^{-11} \mathrm{~m}^{2}(10.132 \mathrm{mD})$ and $k_{f}=1.0 \times 10^{4} k_{m}$, respectively. 

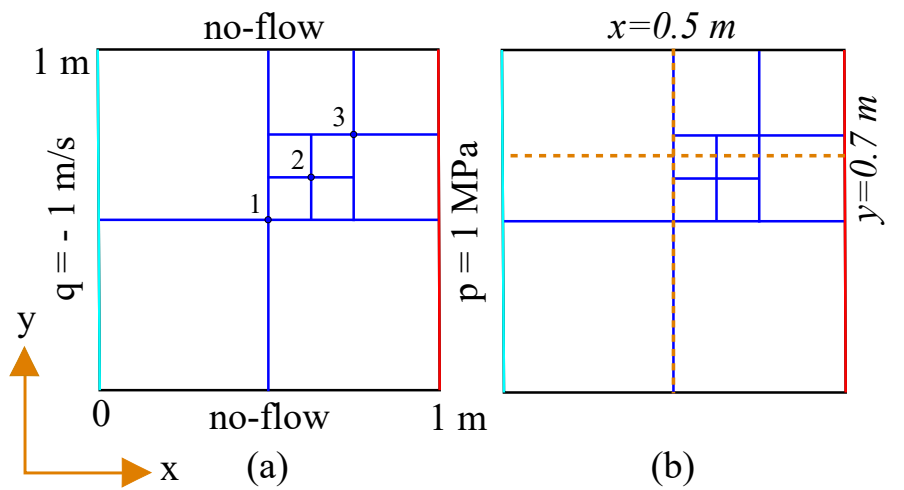

Fracture intersection coordinates

-1. $(1 / 2,1 / 2)$

- 2. $(5 / 8,5 / 8)$

- 3. $(3 / 4,3 / 4)$

Figure 5.15: Regular fracture network: (a) geometry and boundary conditions and (b) cut lines related to the problem.

This problem is subdivided into three main groups named Case 1, Case 2 and Case 3, where each of them is studied by taking into account four coupling meshes. Therefore, a total of 12 analyses are performed, and the following parameter is assumed

$$
R=\frac{s_{m}}{s_{f}}
$$

where $s_{m, f}$ are the average size of the porous medium and fracture elements, respectively. In each case a different parameter was used. For Meshes 1, 2, 3 and 4 of the Case 1 , the value adopted was $R \approx 1.0$; for the Meshes $5,6,7$ and 8 of the Case 2, the parameter adopted was $R \approx 1.5$; and for Meshes $9,10,11$ and 12 of the Case 3 , the parameter adopted was $R=2.0$. Table 5.5 presents the properties of each mesh considered in these analyses. 
Table 5.5: Properties of the meshes employed for the regular fracture network problem.

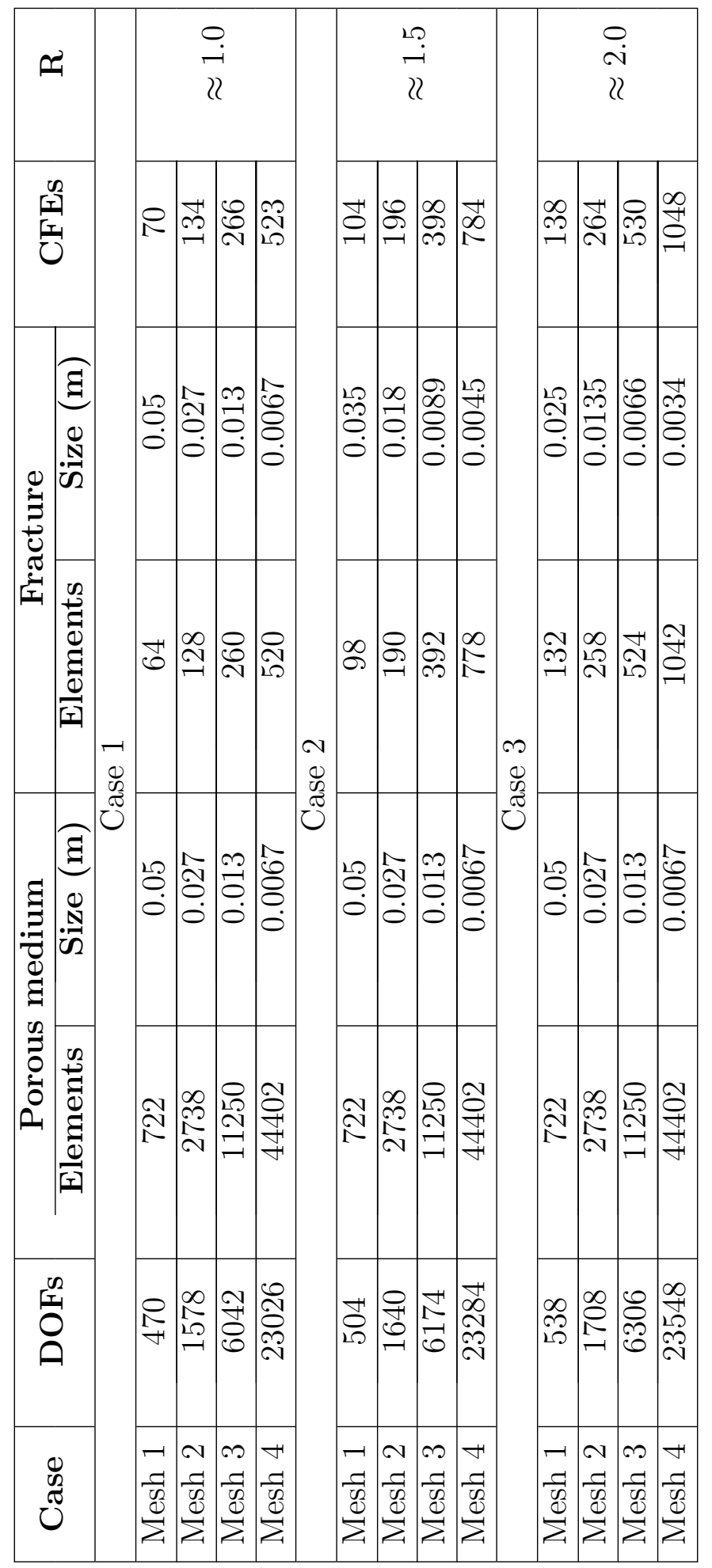

CFEs are used to couple the overlapping non-matching meshes of the porous medium 
and fracture network for all the cases studied. Figure 5.16 illustrates the four meshes adopted for the Case 1; Figure 5.17 illustrates the four meshes adopted for the Case 2; and the Figure 5.18 illustrates the four meshes adopted for the Case 3.

\section{Case 1}

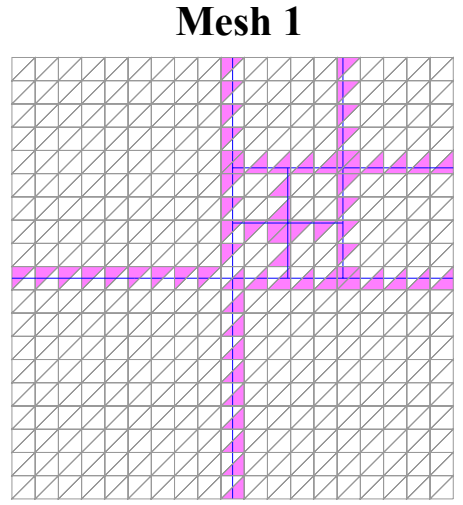

(a)

Mesh 3

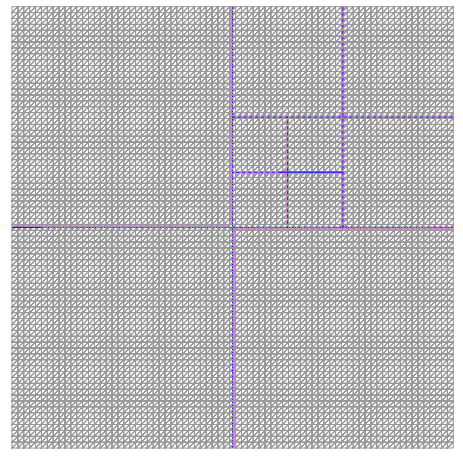

(c)

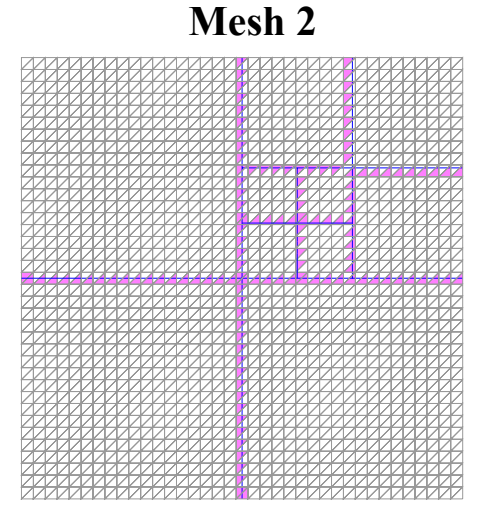

(b)

Mesh 4

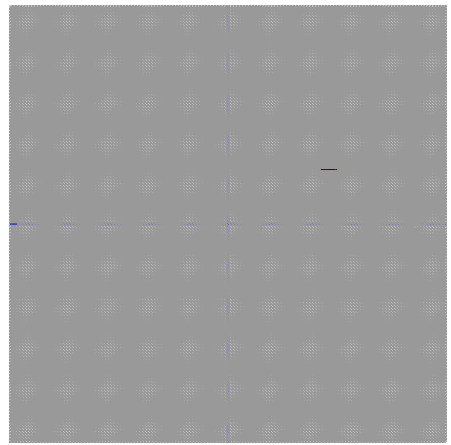

(d)

Figure 5.16: FE meshes used for the Case 1 of the regular fracture network problem: (a) Mesh 1; (b) Mesh 2; (c) Mesh 3; and (d) Mesh 4. 


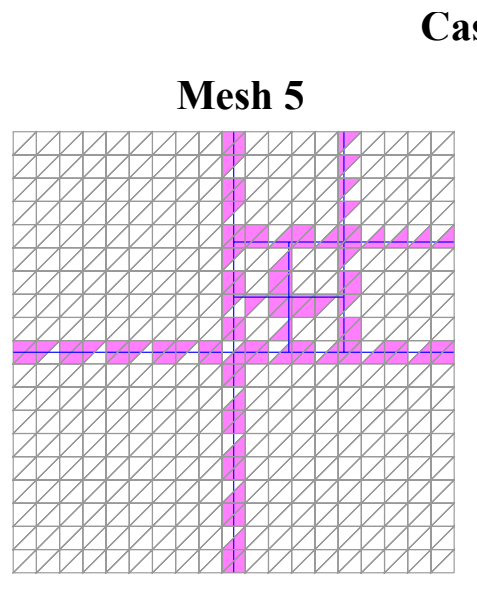

(a)

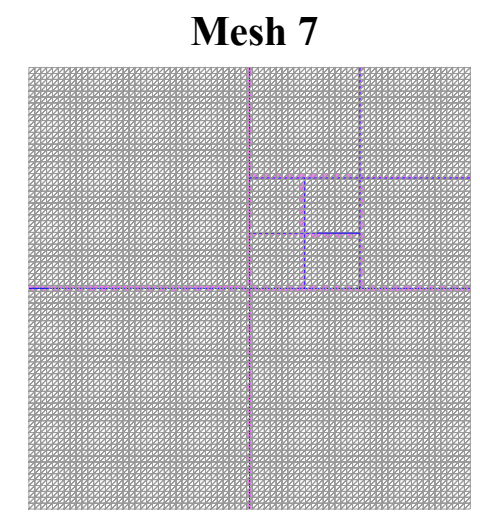

(c)

\section{Case 2}

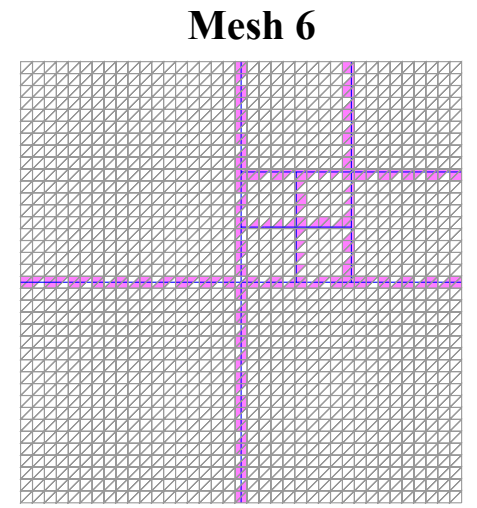

(b)

Mesh 8

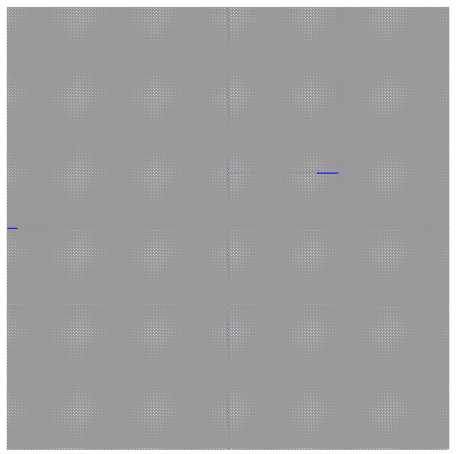

(d)

Figure 5.17: FE meshes used for the Case 2 of the regular fracture network problem: (a) Mesh 5; (b) Mesh 6; (c) Mesh 7; and (d) Mesh 8. 


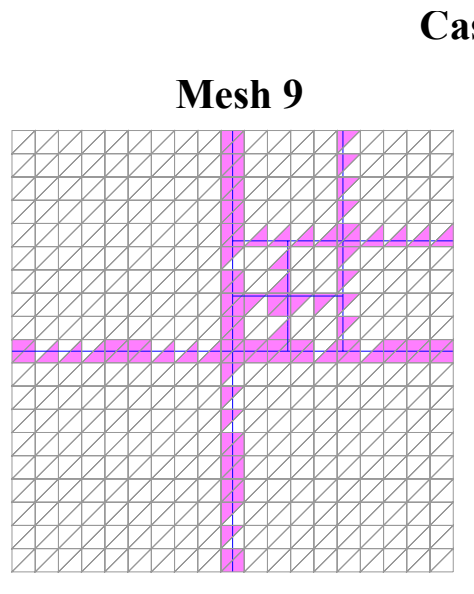

(a)

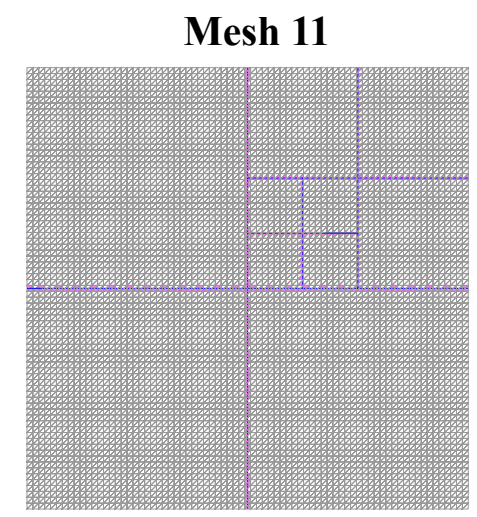

(c)
Case 3

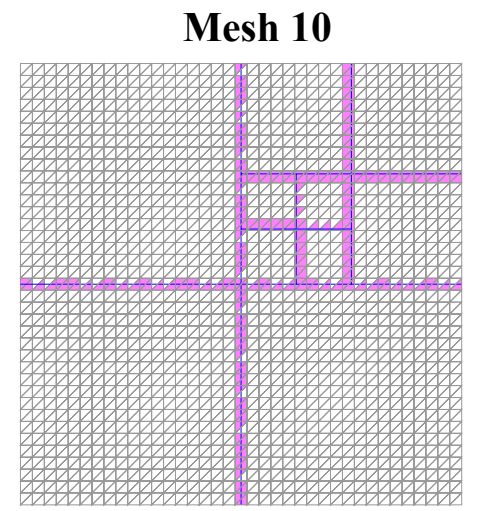

(b)

Mesh 12

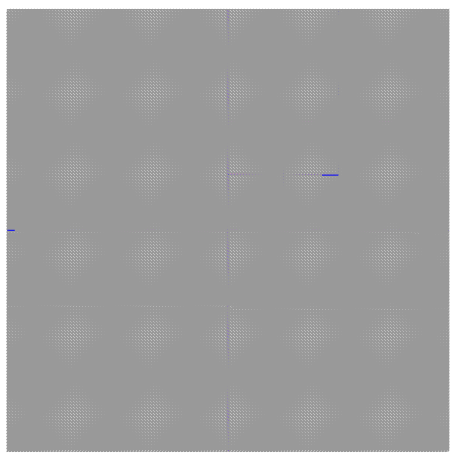

(d)

Figure 5.18: FE meshes used for the Case 3 of the Regular fracture network problem: (a) Mesh 9; (b) Mesh 10; (c) Mesh 11; and (d) Mesh 12. 


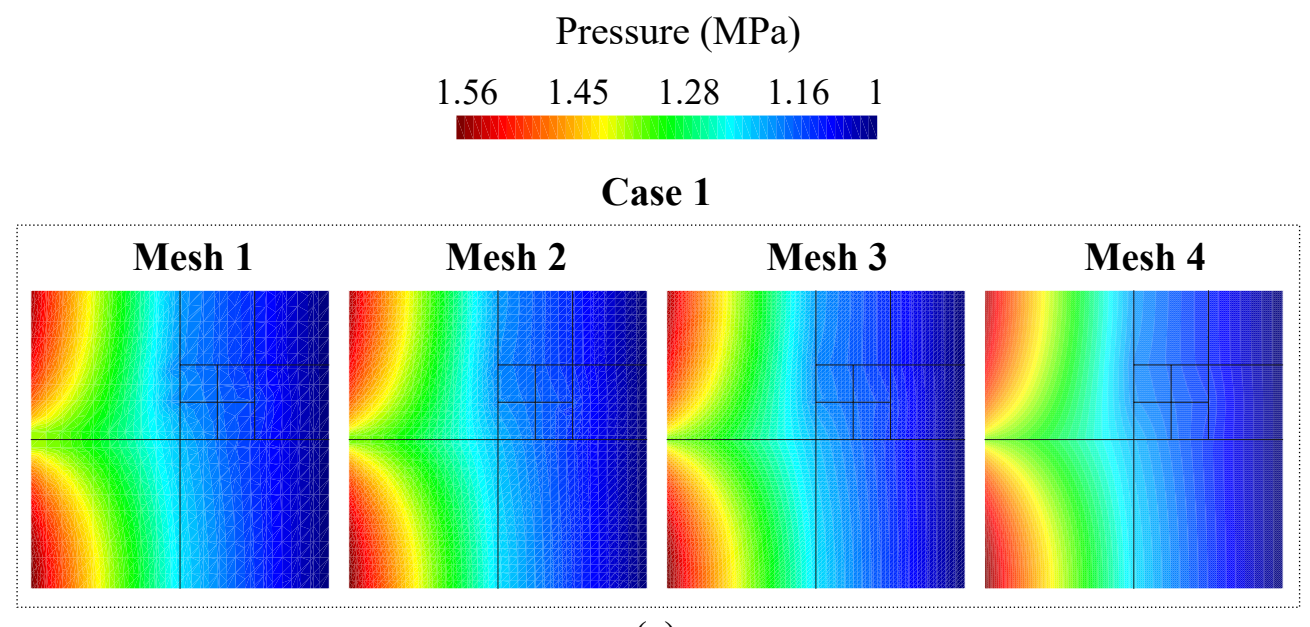

(a)

\section{Case 2}

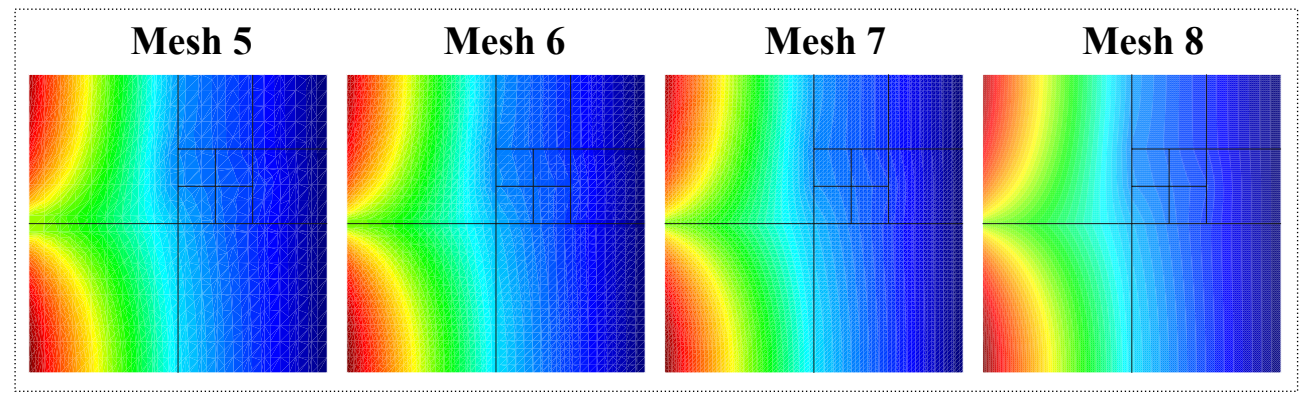

(b)

Case 3

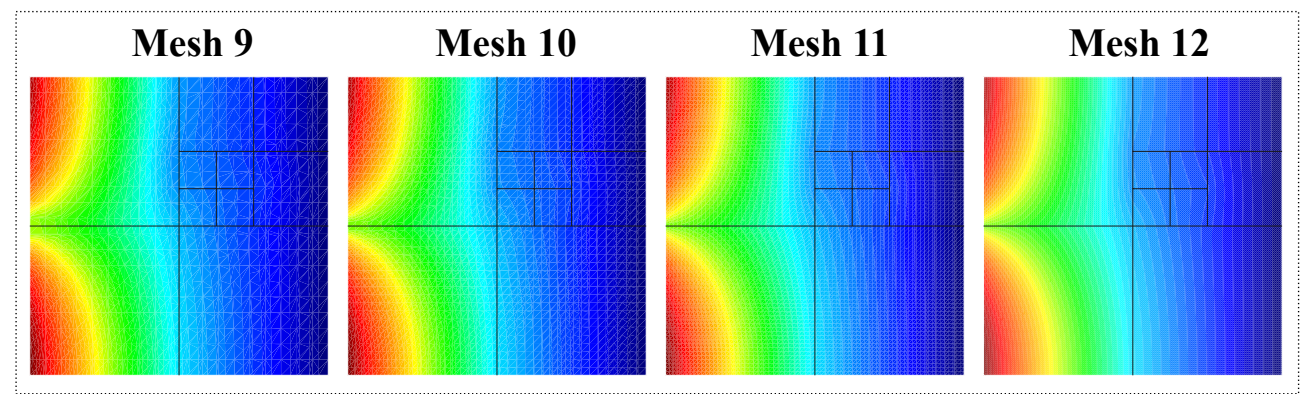

(c)

Figure 5.19: Pressure gradient obtained for the regular fracture network for: (a) Case 1, (b) Case 2 and (c) Case 3.

The solutions of pressure gradient for the Case 1, Case 2 and Case 3 are shown in Figure 5.19, in which a very similar pressure field presented by Flemisch for the same problem.

Figure 5.20 shows a comparison between the reference results presented by Flemisch 
et al. (2018) (which used a mesh with 2352280 DOFs, 1136456 matrix elements and 38600 fracture elements) against the ones obtained via CFEs. Figure 5.20 (a) and (b) present the pressure profile curves for Case 1 (i.e., Meshes 1, 2, 3 and 4) at $x=0.5 \mathrm{~m}$ and $y=0.7 \mathrm{~m}$, respectively. Similarly, Figure 5.20(c) and (d) compare the pressure curves for the Case 2 (i.e., Meshes 5, 6, 7 and 8) with the reference one at $x=0.5 \mathrm{~m}$ and $y=0.7 \mathrm{~m}$, respectively. The responses of the Case 3 (i.e., Meshes 9, 10, 11 and 12) are shown in Figure 5.20(e) and (f) at $x=0.5 \mathrm{~m}$ and $y=0.7 m$, respectively. All results associated with the CFEs show a good agreement with the reference curve, which indicate that the method proposed in this work is able to capture the effects of a fracture network by considering fracture interaction. Moreover, the mesh refinement study conducted in this example showed that the method can be used satisfactorily with coarse meshes.

To ensure the good prediction of numerical results, the Mesh 12 (Case 3) was plotted with eight other methods which used this benchmark (the numerical results are available at: <https://git.iws . unistuttgart. de/benchmarks/fracture-flow). Figure 5.21(a) illustrates the pressure distribution along $x=0.5 \mathrm{~m}$ and Figure 5.21(b) presents the pressure profile at $y=0.7 \mathrm{~m}$. The graphics indicate that the proposed model present one of the best results among the methods available on literature. 


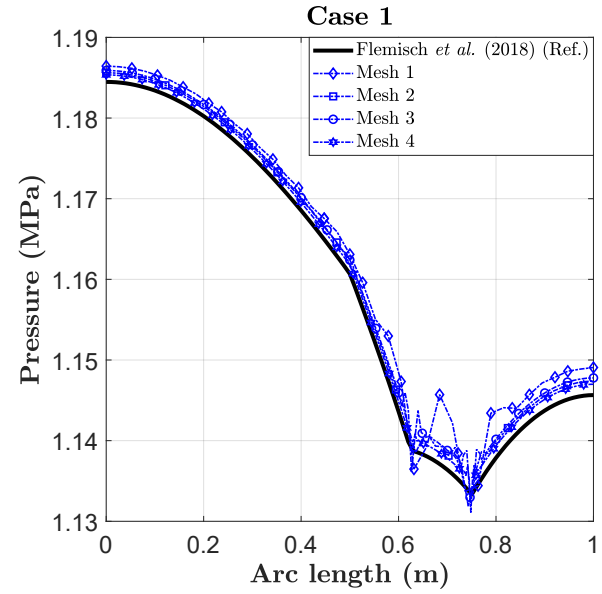

(a)

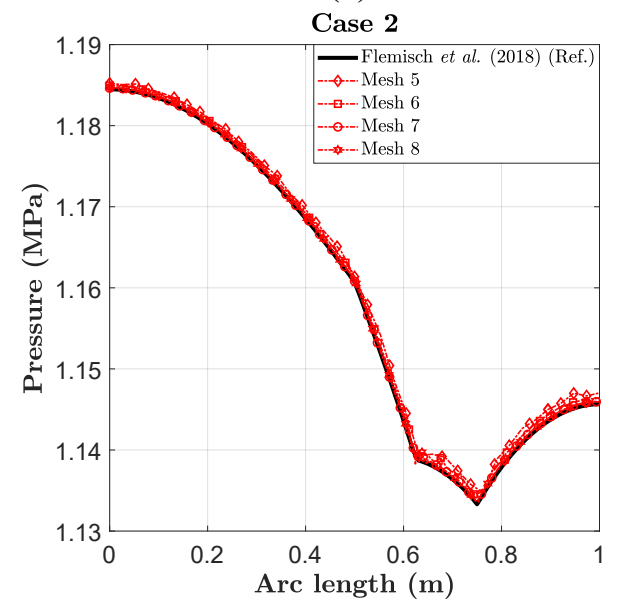

(c)

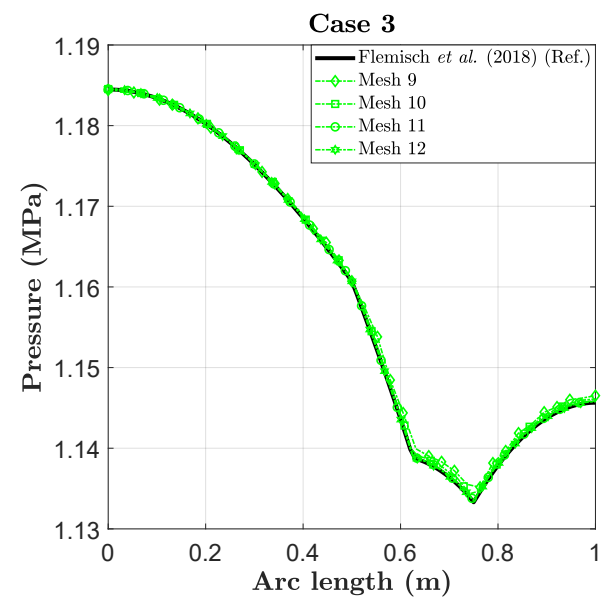

(e)

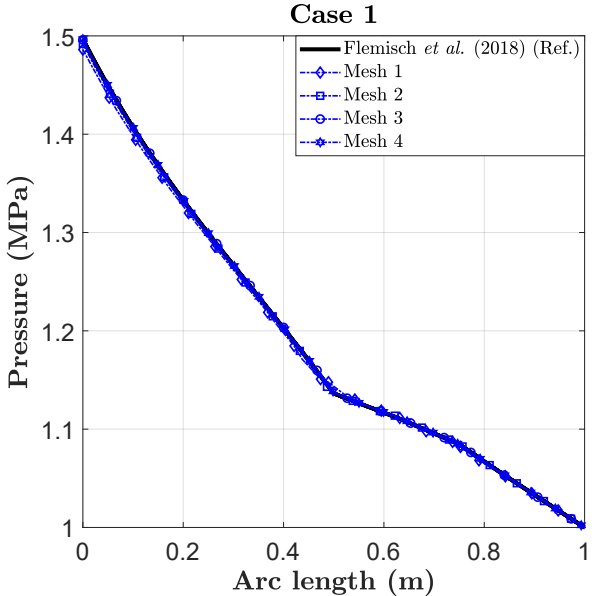

(b)

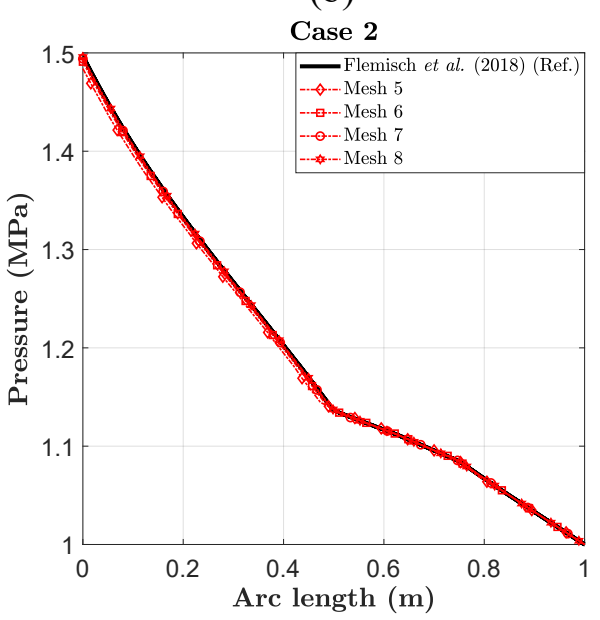

(c)

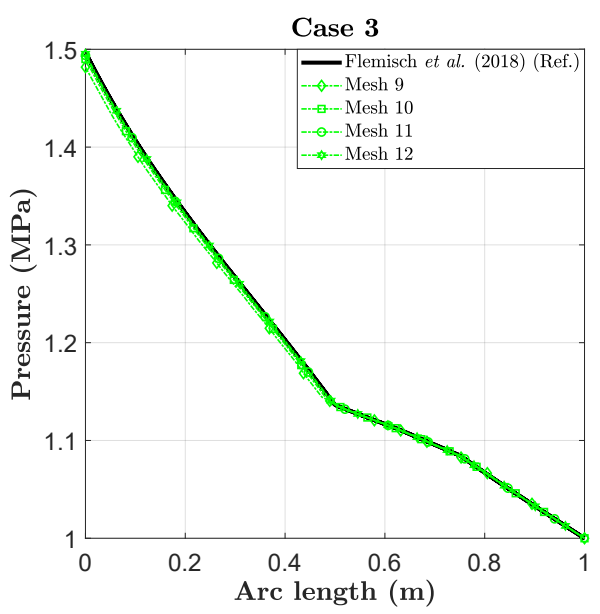

(e)

Figure 5.20: Comparison between the results presented by Flemisch et al. (2018) against that ones via CFEs related to the Case 1 measured in (a) $x=0.5 \mathrm{~m}$ and (b) $y=0.7 \mathrm{~m}$; Case 2 measured in (c) $x=0.5 \mathrm{~m}$ and (d) $y=0.7 \mathrm{~m}$, and Case 3 measured in (e) $x=0.5 \mathrm{~m}$ and (f) $y=0.7 \mathrm{~m}$. 

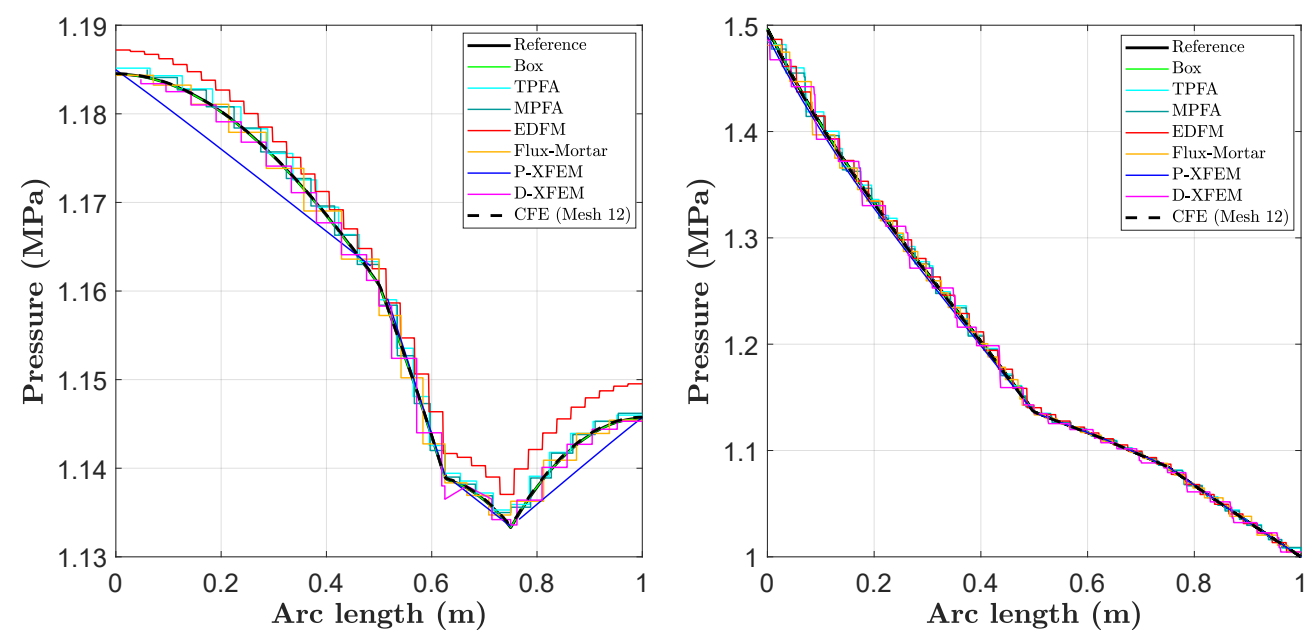

Figure 5.21: Pressure profile of regular fracture network with CFEs and the methods studied by Flemisch et al. (2018).

Figure 5.22 shows the RMS error curves varying according to the number of degree of freedoms. For all the meshes analyzed (i.e. Meshes 1 to 12), the RMS error decreases when increasing the number of DOFs, which is an aspect very common for analyses via FEM, suggesting that the responses are convergent for higher mesh refinement levels. Moreover, Case 3 (associated with $R=2.0$ for Meshes 7 to 12) presents a more significant reduction in the RMS error, and therefore, it is possible to conclude that when the fracture elements (bar elements, in this example) are smaller than the porous matrix ones, the approach tends to be more accurate. Thus, based on this behavior, it is suggested the use of elements that corresponds to at least $R=1.0$. 


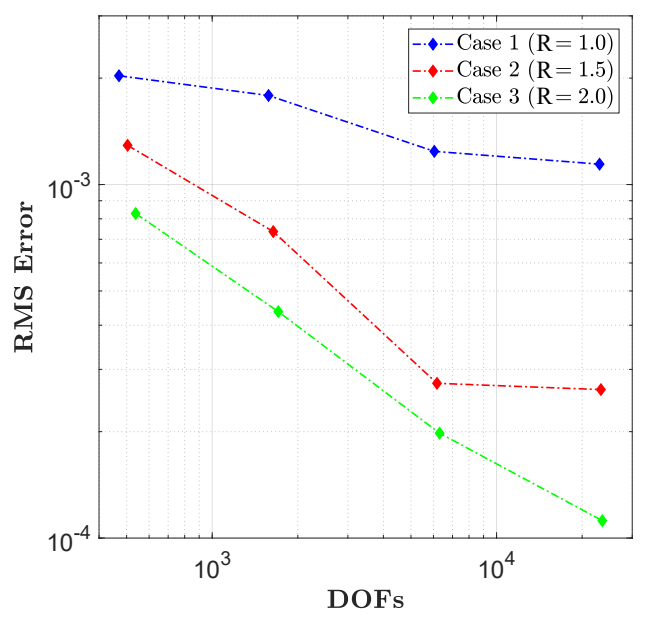

Figure 5.22: RMS error between the reference curve presented by Flemisch and the results obtained via CFE method related to the Cases 1, 2 and 3.

\subsection{Naturally fractured-vuggy reservoirs}

The objective of this example is to demonstrate the capability of the proposed approach to simulate complex problems such as naturally fractured-vuggy reservoirs. Thus, the influence of the connection between fractures and vugs on the pressure distribution is assessed. A total of three conceptual models presented by Zhang et al. (2016) are numerically simulated: fractured porous medium; vuggy porous medium; and fractured-vuggy porous medium.

Figure 5.23 presents the geometry of the three models simulated and the boundary conditions. The top and bottom faces have no-flow Neumann conditions and the left and right sides have Dirichlet conditions of $p_{1}=5 \mathrm{MPa}$ and $p_{2}=0$, respectively. For all the cases, the same geometry of a square plane of domain $\Omega(0,25)^{2}$ and permeability of the porous matrix of $9.36 \times 10^{-13} \mathrm{~m}^{2}(948 \mathrm{mD})$ are considered. The first model is an irregular fracture network that includes a random arrangement of fractures with different orientations and constant aperture of $1 \times 10^{-3} \mathrm{~m}$, as shown in Figure 5.23(a). The second model consists of ellipsoidal and circle vug spaces embedded in the porous medium. Due to limitations of this research, it is assumed that the vug spaces are filled with sand, and Darcy's law is used to describe the fluid flow in their domains, with a permeability of $1.0 \times 10^{-5} \mathrm{~m}^{2}\left(1,0 \times 10^{10} \mathrm{mD}\right)$ (Figure 5.23(b)). The last example is an assembly of the first two examples which contains fractures and vugs (see Figure 5.23(c)). 


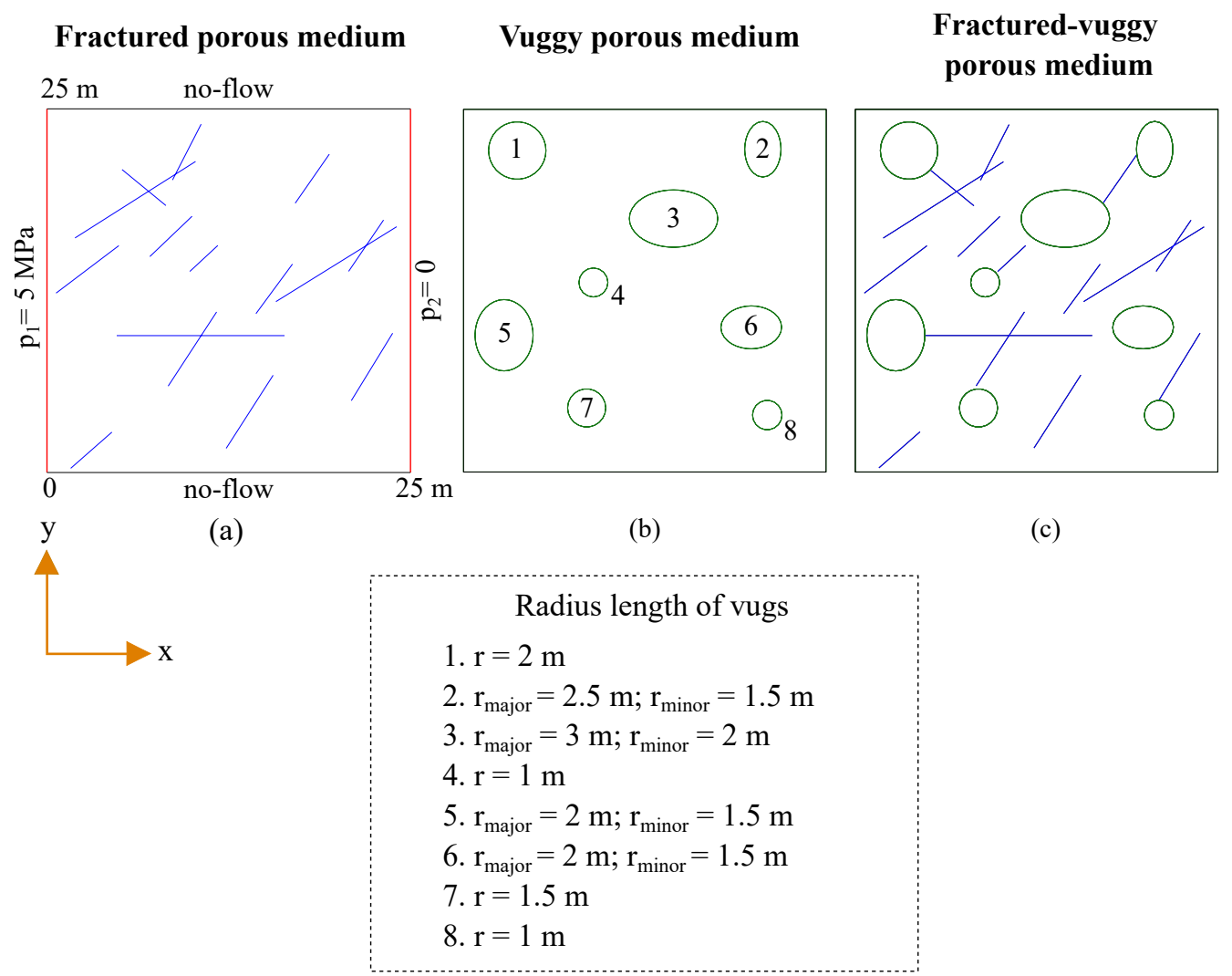

Figure 5.23: Geometry and boundary conditions for the three cases considered: (a) fractured porous medium; (b) vuggy porous medium; and (c) fractured-vuggy porous medium.

The FE discretization for the three cases analyzed are illustrated in Figure 5.24, and the characteristics of FE meshes used to represent porous medium, fractures and vugs are listed in Table 5.6. The FE meshes from porous matrix and vugs are conforming, and three-noded triangular elements are used in the discretization of the vugs. 


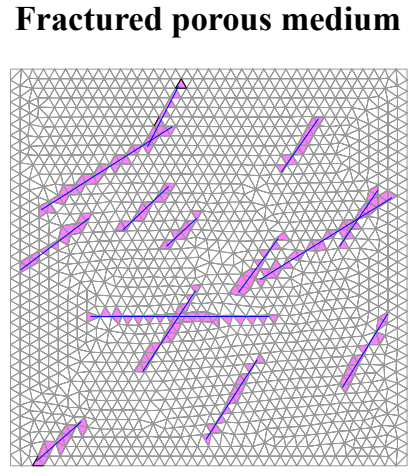

(a)

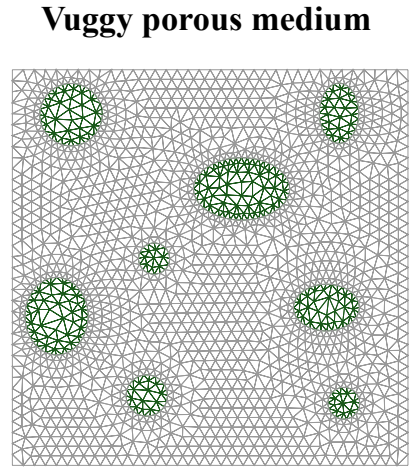

(b)

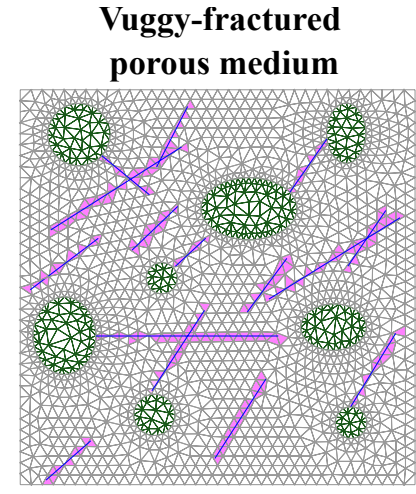

(c)

Figure 5.24: FE mesh discretization with CFEs of conceptual models: (a) fractured porous medium; (b) vuggy porous medium; and (c) fractured-vuggy porous medium.

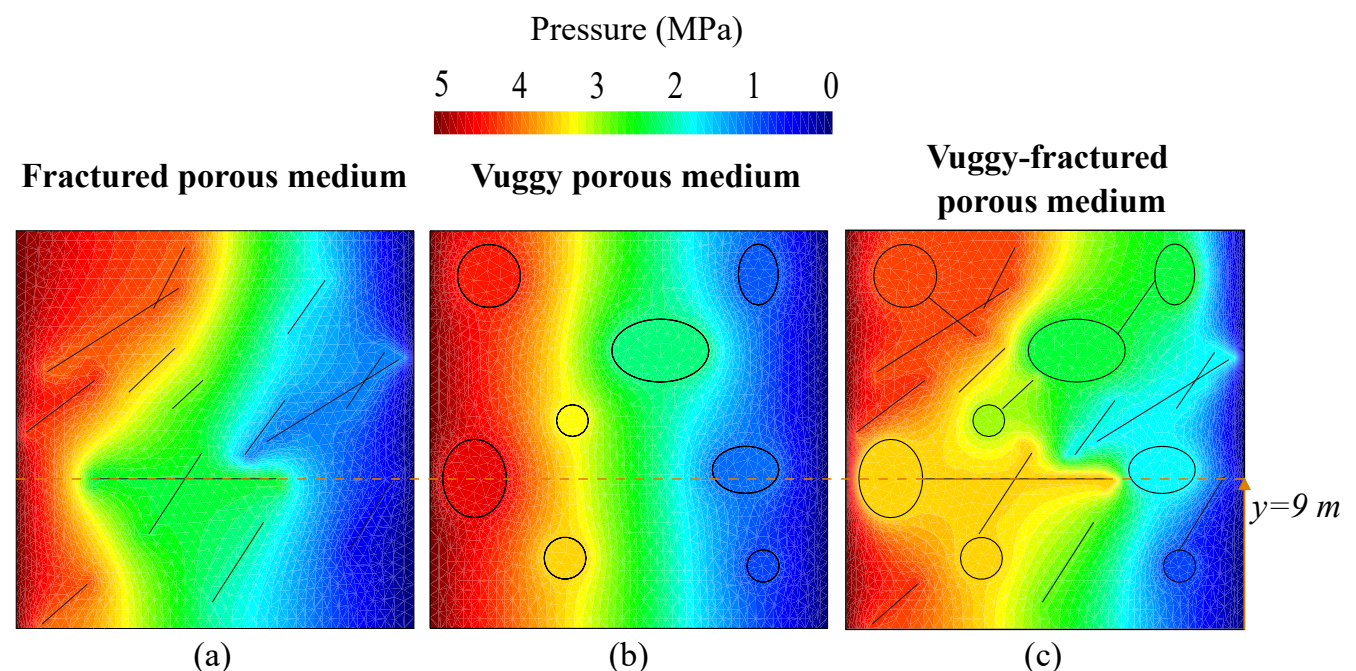

Figure 5.25: Pressure distribution of (a) fractured porous medium; (b) vuggy porous medium; and (c) vuggy-fractured porous medium. 
Table 5.6: Characteristics of the FE meshes used in the numerical models.

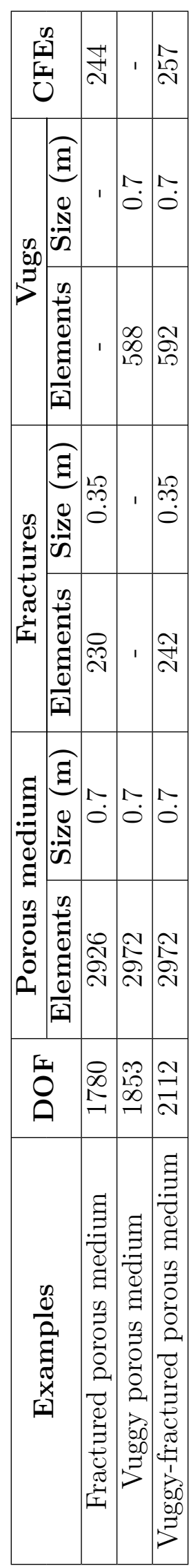


The pressure distribution obtained are presented in Figure 5.25. For the fractured porous medium, the pressure distribution is fully affected by irregular fracture network, especially the horizontal fracture which is parallel to fluid flow vector and acts as the main pathway for fluid flow (see Figure 5.25(a)). For vuggy porous medium, pressure is dissipated quickly, it indicates that vug space acts as a path for fluid due to higher permeability in comparison with fracture permeability, as illustrated in Figure 5.25(b). Following the same behavior, the vuggy-fractured porous medium, presented in Figure 5.25(c), shows that vugs connected with fractures induce the fluid flow through fractures and, rapidly, dissipate pressure distribution in comparison with fractured porous medium. In other words, the vugs act as a fluid path and their behavior is similar of fracture behavior.

With the purpose to clarify the pressure distribution along the domain, Figure 5.26 presents a pressure profile crossing the $y$-axis at $9 \mathrm{~m}$. Due to the continuum behavior of the pressure profiles, it is possible to note that the use of CFEs is able to capture the fluid flow exchange between the fracture and porous medium. Besides, the fracture network and vug spaces greatly influence on drop of pressure close to the boundary. It indicates that fluid flow is dissipated through vugs and fractures, especially for a fractured porous medium.

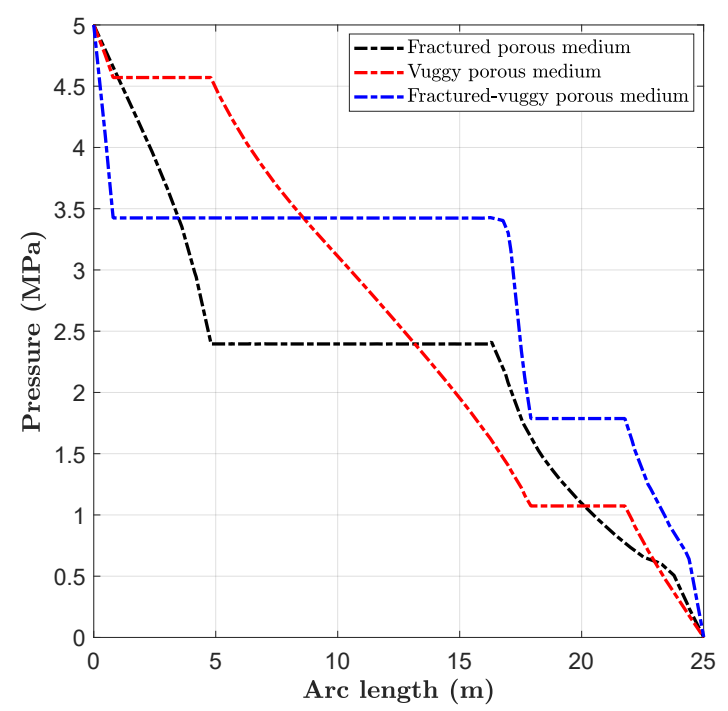

Figure 5.26: Pressure profile for the three cases numerically analyzed along $y=$ $10 \mathrm{~m}$. 


\section{Conclusions}

\subsection{General conclusions}

This work presents a finite element scheme to mimic the hydrostatic behavior of naturally fractured reservoirs by using coupling finite elements (CFEs) to couple initially independent meshes from porous matrix and discrete fracture network. The external nodes of the CFEs coincide with the element nodes of the porous matrix and the extra internal node of the CFEs is associated with one node of the finite element that represents the natural fracture, so that a penalty parameter ensures the continuity of the pressure field between the two meshes. The attractiveness of this technique stems from the fact that the degrees of freedom of problems with non-matching meshes remain the same (i.e., no extra degree of freedom is added to the problem). Moreover, there is no need to refine the mesh in the region near the fracture. The technique is also capable of coupling non-matching meshes of fracture to establish the fracture interaction. Regarding the advantages of the proposed approach when compared with the existing ones available in the literature, the following highlights can be listed:

- Standard finite elements are employed to describe the behavior of both porous matrix and fractures and CFEs are added during the pre-processing stage only to couple the initially independent meshes;

- The formulation behind the CFEs is a simple extension of the traditional finite element, and consequently, they can be easily included in existing FEM codes;

- No additional degree of freedom is added to the problem due to the fact that the CFEs share nodes of both non-matching meshes, i.e. they are constructed based on the existing nodes of the standard finite element mesh;

- No especial integration scheme is required for the introduction of CFEs;

- The proposed methodology is able to tackle complex 2D discrete fracture networks; fractures with any dimension and positioned in any direction can be 
considered.

Five 2D studies were carried out to show the capacity of the proposed technique to simulate the fluid transfer in fractured porous medium. In the first two examples (validation of the proposed model - Section 5.1) the results demonstrated that the use of structured or unstructured meshes does not influence the accuracy of the proposed approach for modeling fluid flow in naturally fractured porous medium. These examples also showed that a penalty parameter of $C=10^{-2} \mathrm{~m} /(\mathrm{MPa}$.s $)$ was able to enforce the continuity of the pressure field between the non-matching meshes from porous matrix and fractures, and the proposed approach is able to capture the effect of the inclination of the fracture. The third and fourth examples (mesh refinement study - Section 5.2) show that even when a coarse mesh is adopted, the proposed approach presents good predictions in terms of pressure distribution. The examples also showed that the model does not require nodes on the intersection between fractures and, the fracture-fracture interaction is automatically captured by the coupling finite elements. Moreover, the model was able to capture the effects of a fracture network by considering fracture interaction, and it is also possible to conclude that when the fracture elements are smaller than the porous matrix ones, the approach tends to be more accurate. In last example (naturally fractured-vuggy reservoirs Section 5.3), the proposed approach demonstrated to be promising for the simulation of complex problems such as naturally fractured-vuggy porous medium. In this example the model was able to capture the effect of the fractures, vugs, and the interaction between them on the pressure distribution.

Finally, the results obtained by the analyses indicate that the proposed finite element coupling technique is able to properly reproduce the fluid flow mechanisms in reservoirs with both simple and complex fracture networks. It is worthwhile to comment that the porous rock and fracture meshes consist of standard finite elements and the matrix-fracture interaction is considered by simply adding CFEs.

\subsection{Recommendations for future research}

The numerical analyses demonstrated that the proposed coupling scheme is able to represent explicitly discrete fractures in the numerical modeling of fluid flow in naturally fractured porous medium. Thus, based on the promising results obtained in this research, some recommendations for future research as listed below: 
- To extend the proposed formulation for 3D analyses.

- To consider hydrodynamic fluid flow in naturally fractured reservoirs.

- To include the transverse permeability of the discontinuities in the pressure distribution.

- To include mechanical and thermal effects for the simulations of practical cases. 


\section{Bibliography}

Abdassah, D., Ershaghi, I., et al. Triple-porosity systems for representing naturally fractured reservoirs. SPE Formation Evaluation, 1(02):113-127, 1986.

Aguilera, R. F. et al. A triple porosity model for petrophysical analysis of naturally fractured reservoirs. Petrophysics, 45(02), 2004.

Al-Ahmadi, H. A., Wattenbarger, R. A., et al. Triple-porosity models: one further step towards capturing fractured reservoirs heterogeneity. 2011.

Alyafei, N. Fundamentals of reservoir rock properties. 2019.

Annavarapu, C., Hautefeuille, M., and Dolbow, J. E. A robust nitsche's formulation for interface problems. Computer Methods in Applied Mechanics and Engineering, 225-228:44 - 54, 2012. ISSN 0045-7825. doi: 10.1016/j.cma.2012.03.008. URL https://doi.org/10.1016/j.cma.2012.03.008.

Bear, J. Dynamics of fluids in porous media. American Elsevier Publishing Company, Inc., 1972.

Bear, J. and Bachmat, Y. Theory and applications of transport in porous media. page $554,1990$.

Becker, R., Hansbo, P., and Stenberg, R. A finite element method for domain decomposition with non-matching grids. ESAIM: Mathematical Modelling and Numerical Analysis - Modélisation Mathématique et Analyse Numérique, 37(2): 209-225, 2003. doi: 10.1051/m2an:2003023. URL https://doi.org/10.1051/ m2an: 2003023.

Berre, I., Doster, F., and Keilegavlen, E. Flow in fractured porous media: a review of conceptual models and discretization approaches. Transport in Porous Media, 130(1):215-236, 2019.

Bitencourt Jr, L. A., Manzoli, O. L., Prazeres, P. G., , E. A., and Bittencourt, T. N. A coupling technique for non-matching finite element meshes. Computer Methods in Applied Mechanics and Engineering, 290:19-44, 2015. 
Bitencourt Jr, L. A., Manzoli, O. L., Trindade, Y. T., Rodrigues, E. A., and Dias-da Costa, D. Modeling reinforced concrete structures using coupling finite elements for discrete representation of reinforcements. Finite Elements in Analysis and Design, 149:32-44, 2018.

Bitencourt Jr, L. A., Manzoli, O. L., Bittencourt, T. N., and Vecchio, F. J. Numerical modeling of steel fiber reinforced concrete with a discrete and explicit representation of steel fibers. International Journal of Solids and Structures, 159: 171-190, 2019.

Březina, J. and Exner, P. Fast algorithms for intersection of non-matching grids using plücker coordinates. Computers $\&$ Mathematics with Applications, 74(1):174 - 187, 2017. ISSN 0898-1221. doi: 10.1016/j.camwa.2017.01.028. URL https:// doi.org/10.1016/j.camwa.2017.01.028. 5th European Seminar on Computing ESCO 2016.

Byrne, D. J., Barry, P., Lawson, M., and Ballentine, C. Noble gases in conventional and unconventional petroleum systems. Geological Society, London, Special Publications, 468(1):127-149, 2018.

Chai, Z., Yan, B., Killough, J., and Wang, Y. An efficient method for fractured shale reservoir history matching: The embedded discrete fracture multi-continuum approach. Journal of Petroleum Science and Engineering, 160:170-181, 2018.

Chen, H., Li, H., Li, Z., Li, S., Wang, Y., Wang, J., and Li, B. Effects of matrix permeability and fracture on production characteristics and residual oil distribution during flue gas flooding in low permeability/tight reservoirs. Journal of Petroleum Science and Engineering, 195, 2020.

Chengzao, J., Min, Z., and Zhang, Y. Unconventional hydrocarbon resources in china and the prospect of exploration and development. Petroleum Exploration and Development, 39(2):139-146, 2012.

Chung, E. T., Efendiev, Y., Leung, W. T., Vasilyeva, M., and Wang, Y. Non-local multi-continua upscaling for flows in heterogeneous fractured media. Journal of Computational Physics, 372.

Coats, K. H. et al. Implicit compositional simulation of single-porosity and dualporosity reservoirs. In SPE symposium on reservoir simulation. Society of Petroleum Engineers, 1989.

Coussy, O. Poromechanics. John Wiley and Sons, 2004. 
Cundall, P. and Hart, R. Numerical modelling of discontinua. Engineering Computations, pages 101-113, 1992.

Damirchi, B. V., Carvalho, M. R., Bitencourt Jr., L. A., Manzoli, O. L., and Dias-da Costa, D. Transverse and longitudinal fluid flow modelling in fractured porous media with non-matching meshes. International Journal for Numerical and Analytical Methods in Geomechanics, 2020.

Dandekar, A. Y. Petroleum reservoir rock and fluid properties. CRC press, 2013.

Darcy, H. Les fontaines publiques de la ville de Dijon: exposition et application... 1856.

Dhia, H. B. and Rateau, G. The arlequin method as a flexible engineering design tool. International Journal for Numerical Methods in Engineering, 62(11):14421462, 2005. doi: 10.1002/nme.1229. URL https://doi .org/10.1002/nme.1229.

Dietrich, P., Helmig, R., Hötzl, H., Sauter, M., Köngeter, J., and Teutsch, G. Flow and transport in fractured porous media. Springer Science \& Business Media, 2005.

Efendiev, Y., Galvis, J., and Hou, T. Y. Generalized multiscale finite element methods (gmsfem). Journal of Computational Physics, 251:116-135, 2013.

Etnyre, L. M. Finding oil and gas from well logs. Springer, 1989.

Fabbri, H. A., Cleto, P. R., Gaiotto Jr, A. T., Rodrigues, E. A., Maedo, M. A., and Manzoli, O. L. Modeling the closure behavior of natural fractures in porous media using high aspect ratio interface elements. Journal of Petroleum Science and Engineering, 196, 2021.

Fanchi, J. R. and Christiansen, R. L. Introduction to petroleum engineering. Wiley Online Library, 2017.

Fang, S., Cheng, L., and Ayala, L. F. A coupled boundary element and finite element method for the analysis of flow through fractured porous media. Journal of Petroleum Science and Engineering, 152:375-390, 2017.

Flemisch, B., Berre, I., Boon, W., Fumagalli, A., Schwenck, N., Scotti, A., Stefansson, I., and Tatomir, A. Benchmarks for single-phase flow in fractured porous media. Advances in Water Resources, 111:239-258, 2018.

Geiger, S., Dentz, M., Neuweiler, I., et al. A novel multi-rate dual-porosity model for improved simulation of fractured and multiporosity reservoirs. SPE journal, 18(04):670-684, 2013. 
Hajibeygi, H., Tchelepi, H. A., et al. Compositional multiscale finite-volume formulation. SPE Journal, 19(02):316-326, 2014.

Hill, A., Thomas, G., et al. A new approach for simulating complex fractured reservoirs. In Middle east oil technical conference and exhibition. Society of Petroleum Engineers, 1985.

Hu, M., Rutqvist, J., and Wang, Y. A practical model for fluid flow in discretefracture porous media by using the numerical manifold method. Advances in water resources, 97:38-51, 2016.

Huang, N., Jiang, Y., Liu, R., Li, B., and Sugimoto, S. A novel three-dimensional discrete fracture network model for investigating the role of aperture heterogeneity on fluid flow through fractured rock masses. International Journal of Rock Mechanics and Mining Sciences, 116:25-37, 2019.

Jacob, F. and Ted, B. A first course in finite elements. Wiley, 2007.

Jiang, J. and Younis, R. M. An improved projection-based embedded discrete fracture model (pedfm) for multiphase flow in fractured reservoirs. Advances in Water Resources, 109:267-289, 2017.

Karimi-Fard, M., Durlofsky, L. J., Aziz, K., et al. An efficient discrete fracture model applicable for general purpose reservoir simulators. In SPE Reservoir Simulation Symposium. Society of Petroleum Engineers, 2003.

Kazemi, H., Merrill Jr, L., Porterfield, K., Zeman, P., et al. Numerical simulation of water-oil flow in naturally fractured reservoirs. Society of Petroleum Engineers Journal, 16(06):317-326, 1976.

Leffler, W. L., Pattarozzi, R., and Sterling, G. Deepwater petroleum exploration and production: a nontechnical guide. PennWell Books, 2011.

Li, B., Jiang, Y., Koyama, T., Jing, L., and Tanabashi, Y. Experimental study of the hydro-mechanical behavior of rock joints using a parallel-plate model containing contact areas and artificial fractures. International Journal of Rock Mechanics and Mining Sciences, 45(3):362-375, 2008.

Li, T., Gao, Y., Han, D., Yang, F., and Yu, B. A novel pod reduced-order model based on edfm for steady-state and transient heat transfer in fractured geothermal reservoir. International Journal of Heat and Mass Transfer, 146:118783, 2020. ISSN 0017-9310. doi: https://doi.org/10.1016/j.ijheatmasstransfer.2019.118783. URL https://doi.org/10.1016/j.ijheatmasstransfer.2019.118783. 
Li, Y. Development theories and methods of fracture-vug carbonate reservoirs. Academic Press, 2017.

Lie, K.-A. and Mallison, B. T. Mathematical models for oil reservoir simulation. Encyclopedia of Applied and Computational Mathematics, 1:8, 2013.

Liu, J., Wang, J., Gao, F., Leung, C. F., and Ma, Z. A fully coupled fracture equivalent continuum-dual porosity model for hydro-mechanical process in fractured shale gas reservoirs. Computers and Geotechnics, 106:143-160, 2019.

Liu, L., Yao, J., Zhang, L., An, S., Zhao, J., and Sun, H. Rev-scale simulation of micro-fractured unconventional gas reservoir. Journal of Natural Gas Science and Engineering, 48:100-110, 2017.

Liu, R., Li, B., and Jiang, Y. Critical hydraulic gradient for nonlinear flow through rock fracture networks: The roles of aperture, surface roughness, and number of intersections. Advances in Water Resources, 88:53-65, 2016.

Long, J., Remer, J., Wilson, C., and Witherspoon, P. Porous media equivalents for networks of discontinuous fractures. Water Resources Research, 18(3):645-658, 1982.

Lyons, W. C. and Plisga, G. J. Standard handbook of petroleum and natural gas engineering. Elsevier, 2011.

Ma, Y. Z. and Holditch, S. Unconventional oil and gas resources handbook: Evaluation and development. Gulf professional publishing, 2015.

Manzoli, O. L., Borges, L. F., Rodrigues, E. A., Cleto, P. R., Maedo, M. A., and Jr., L. A. B. A new discrete fracture approach based on the use of coupling finite elements for modeling fluid transport in naturally fractured porous media. Computer Methods in Applied Mechanics and Engineering, 386:114112, 2021. ISSN 0045-7825. doi: https://doi.org/10.1016/j.cma.2021.114112. URL https: //www.sciencedirect.com/science/article/pii/S0045782521004436.

Moench, A. F. Double-porosity models for a fissured groundwater reservoir with fracture skin. Water Resources Research, 20(7):831-846, 1984.

Moinfar, A., Narr, W., Hui, M.-H., Mallison, B. T., Lee, S. H., et al. Comparison of discrete-fracture and dual-permeability models for multiphase flow in naturally fractured reservoirs. 2011.

Presho, M. and Hill, M. A conservative generalized multiscale finite volume/element 
method for modeling two-phase flow with capillary pressure. Journal of Computational and Applied Mathematics, page 113026, 2020.

Pruess, K. and Narasimhan, T. A practical method for modeling fluid and heat flow in fractured porous media. 1982.

Rao, X., Cheng, L., Cao, R., Jia, P., Liu, H., and Du, X. A modified projection-based embedded discrete fracture model (pedfm) for practical and accurate numerical simulation of fractured reservoir. Journal of Petroleum Science and Engineering, $187,2020$.

Regnet, J., David, C., Robion, P., and Menendez, B. Microstructures and physical properties in carbonate rocks: a comprehensive review. Marine and Petroleum Geology, 2019.

Rodrigues, E. A., Manzoli, O. L., Bitencourt Jr, L. A., Bittencourt, T. N., and Sánchez, M. An adaptive concurrent multiscale model for concrete based on coupling finite elements. Computer Methods in Applied Mechanics and Engineering, 328:26-46, 2018.

Rodrigues, E. A., Manzoli, O. L., and Bitencourt Jr, L. A. 3d concurrent multiscale model for crack propagation in concrete. Computer Methods in Applied Mechanics and Engineering, 361:112813, 2020.

Schädle, P., Zulian, P., Vogler, D., Bhopalam, S. R., Nestola, M. G., Ebigbo, A., Krause, R., and Saar, M. O. 3d non-conforming mesh model for flow in fractured porous media using lagrange multipliers. Computers \& Geosciences, 132:42 - 55, 2019. ISSN 0098-3004. doi: 10.1016/j.cageo.2019.06.014. URL https://doi . org/10.1016/j.cageo.2019.06.014.

Segura, J. and Carol, I. On zero-thickness interface elements for diffusion problems. International journal for numerical and analytical methods in geomechanics, 28 (9):947-962, 2004.

Sokolova, I., Bastisya, M. G., and Hajibeygi, H. Multiscale finite volume method for finite-volume-based simulation of poroelasticity. Journal of Computational Physics, 379:309-324, 2019.

Song, Y., Li, Z., Jiang, L., and Hong, F. The concept and the accumulation characteristics of unconventional hydrocarbon resources. Petroleum Science, 12(4): 563-572, 2015. 
Spiridonov, D., Vasilyeva, M., and Leung, W. T. A generalized multiscale finite element method (gmsfem) for perforated domain flows with robin boundary conditions. Journal of Computational and Applied Mathematics, 357:319-328, 2019.

Thomas, J. E. Fundamentos da engenharia de petroleo. Interciencia, 2004.

Tiab, D. and Donaldson, E. C. Petrophysics: theory and practice of measuring reservoir rock and fluid transport properties. Gulf professional publishing, 2015.

Trindade, Y. T., Bitencourt Jr, L. A., and Manzoli, O. L. Design of sfrc members aided by a multiscale model: Part ii-predicting the behavior of rc-sfrc beams. Composite Structures, 241:112079, 2020.

Vasilyeva, M., Chung, E. T., Cheung, S. W., Wang, Y., and Prokopev, G. Nonlocal multicontinua upscaling for multicontinua flow problems in fractured porous media. Journal of Computational and Applied Mathematics, 355:258-267, 2019.

Vedachalam, N., Srinivasalu, S., Rajendran, G., Ramadass, G., and Atmanand, M. Review of unconventional hydrocarbon resources in major energy consuming countries and efforts in realizing natural gas hydrates as a future source of energy. Journal of Natural Gas Science and Engineering, 26:163-175, 2015.

Wang, C., Ran, Q., and Wu, Y.-S. Robust implementations of the 3d-edfm algorithm for reservoir simulation with complicated hydraulic fractures. Journal of Petroleum Science and Engineering, 181, 2019.

Warren, J., Root, P. J., et al. The behavior of naturally fractured reservoirs. Society of Petroleum Engineers Journal, 3(03):245-255, 1963.

Wohlmuth, B. I. A mortar finite element method using dual spaces for the lagrange multiplier. SIAM Journal on Numerical Analysis, 38(3):989-1012, 2001. ISSN 00361429. doi: 10.1137/S0036142999350929. URL https://doi.org/10.1137/ S0036142999350929.

Wu, Y.-S. Multiphase fluid flow in porous and fractured reservoir. Elsevier, 2016.

Wu, Y.-S., Pruess, K., et al. A multiple-porosity method for simulation of naturally fractured petroleum reservoirs. SPE Reservoir Engineering, 3(01):327-336, 1988.

Wu, Y.-S., Di, Y., Kang, Z., and Fakcharoenphol, P. A multiple-continuum model for simulating single-phase and multiphase flow in naturally fractured vuggy reservoirs. Journal of Petroleum Science and Engineering, 78(1):13-22, 2011.

Xu, Y., Lima, I., Marcondes, F., and Sepehrnoori, K. Development of an embedded discrete fracture model for $2 \mathrm{~d}$ and $3 \mathrm{~d}$ unstructured grids using an element-based 
finite volume method. Journal of Petroleum Science and Engineering, 195:107725, 2020 .

Yan, B., Alfi, M., An, C., Cao, Y., Wang, Y., and Killough, J. E. General multiporosity simulation for fractured reservoir modeling. Journal of Natural Gas Science and Engineering, 33:777-791, 2016a.

Yan, X., Huang, Z., Yao, J., Li, Y., and Fan, D. An efficient embedded discrete fracture model based on mimetic finite difference method. Journal of Petroleum Science and Engineering, 145:11-21, 2016b.

Zeng, Q., Liu, W., Yao, J., and Liu, J. A phase field based discrete fracture model (pfdfm) for fluid flow in fractured porous media. Journal of Petroleum Science and Engineering, page 107191, 2020.

Zhang, C., Ranjith, P., Perera, M., Li, X., and Zhao, J. Simulation of flow behaviour through fractured unconventional gas reservoirs considering the formation damage caused by water-based fracturing fluids. Journal of Natural Gas Science and Engineering, 57:100-121, 2018.

Zhang, N., Yao, J., Xue, S., and Huang, Z. Multiscale mixed finite element, discrete fracture-vug model for fluid flow in fractured vuggy porous media. International Journal of Heat and Mass Transfer, 96:396-405, 2016.

Zimmerman, R. W. and Bodvarsson, G. S. Hydraulic conductivity of rock fractures. Transport in porous media, 23(1):1-30, 1996. 